\title{
Post-AGB stars with hot circumstellar dust: binarity of the low-amplitude pulsators ${ }^{\star \star \star} \star$
}

\author{
H. Van Winckel ${ }^{1}$, T. Lloyd Evans ${ }^{2}$, M. Briquet ${ }^{1, \star \star \star}$, P. De Cat ${ }^{1,3}$, P. Degroote ${ }^{1}$, W. De Meester ${ }^{1}$, J. De Ridder ${ }^{1 * *}$, \\ P. Deroo $^{1,4}$, M. Desmet ${ }^{1}$, R. Drummond ${ }^{1,5}$, L. Eyer ${ }^{1,6}$, M. A. T. Groenewegen ${ }^{1,3}$, K. Kolenberg ${ }^{1,7}$, D. Kilkenny ${ }^{9}$, \\ D. Ladjal ${ }^{1}$, K. Lefever ${ }^{1,5}$, T. Maas ${ }^{1}$, F. Marang ${ }^{10}$, P. Martinez ${ }^{10}$, R. H. Østensen ${ }^{1}$, G. Raskin ${ }^{1}$, M. Reyniers ${ }^{1,8}$, \\ P. Royer ${ }^{1}$, S. Saesen ${ }^{1, \dagger}$, K. Uytterhoeven ${ }^{1,11}$, J. Vanautgaerden ${ }^{1}$, B. Vandenbussche ${ }^{1}$, F. van Wyk ${ }^{10}$, M. Vučković ${ }^{1}$, \\ C. Waelkens ${ }^{1}$, and W. Zima ${ }^{1}$
}

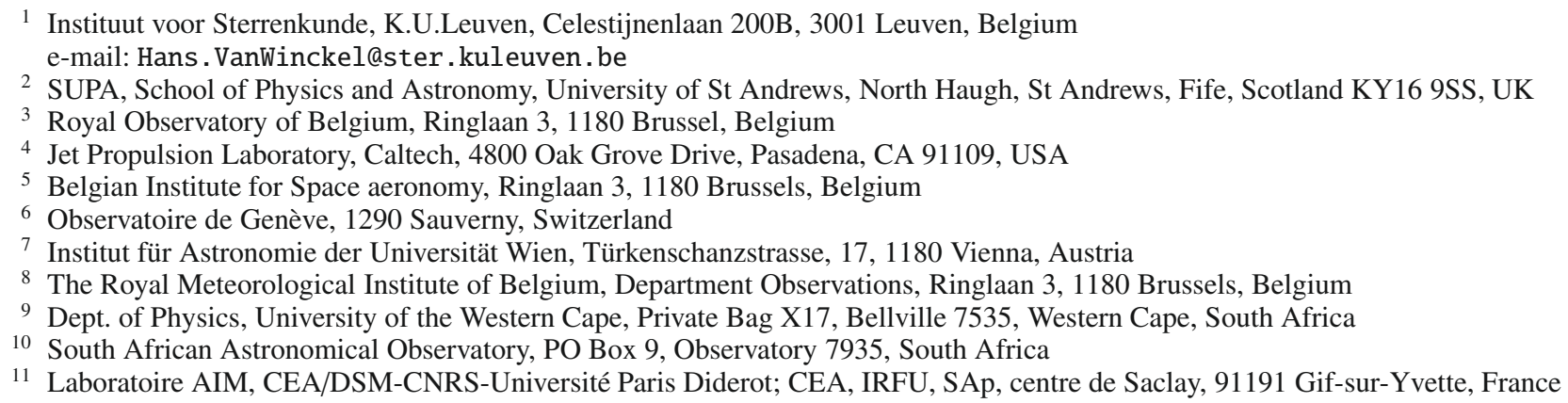

Received 15 April 2009 / Accepted 3 July 2009

ABSTRACT

\begin{abstract}
Context. The influence of binarity on the late stages of stellar evolution remains an open issue.
Aims. While the first binary post-AGB stars were serendipitously discovered, the distinct characteristics of their spectral energy distribution (SED) allowed us to launch a more systematic search for binaries. We selected post-AGB objects, which exhibit a broad dust excess starting either at $\mathrm{H}$ or $\mathrm{K}$, pointing to the presence of a gravitationally bound dusty disc in the system. We initiated an extensive multiwavelength study of those systems and here report on our radial velocity and photometric monitoring results for six stars of early $\mathrm{F}$ type, which are pulsators of small amplitude.

Methods. To determine the radial velocity of low signal-to-noise ratio time-series data, we constructed dedicated autocorrelation masks based on high signal-to-noise ratio spectra, used in our published chemical studies. The radial velocity variations were analysed in detail to differentiate between pulsational variability and variability caused by orbital motion. When available, the photometric monitoring data were used to complement the time series of radial velocity data and to establish the nature of the pulsation. Finally, orbital minimalisation was performed to constrain the orbital elements.

Results. All of the six objects are binaries with orbital periods ranging from 120 to 1800 days. Five systems have non-circular orbits. The mass functions range from 0.004 to $0.57 M_{\odot}$ and the companions are probably unevolved objects of (very) low initial mass. We argue that these binaries must have evolved through a phase of strong binary interaction when the primary was a cool supergiant. Although the origin of the circumstellar disc is not well understood, the disc is generally believed to have formed during this strong interaction phase. The eccentric orbits of these highly evolved objects remain poorly understood. In one object, the line-of-sight grazes the edge of the puffed-up inner rim of the disc.

Conclusions. These results corroborate our earlier statement that evolved objects in binary stars create a Keplerian dusty circumbinary disc. With the measured orbits and mass functions, we conclude that the circumbinary discs seem to have a major impact on the evolution of a significant fraction of binary systems.
\end{abstract}

Key words. stars: AGB and post-AGB - stars: binaries: general - stars: binaries: spectroscopic - stars: chemically peculiar stars: evolution

^ based on observations collected with the Flemish $1.2 \mathrm{~m}$ Mercator telescope at Roque de los Muchachos (Spain), the Swiss $1.2 \mathrm{~m}$ Euler telescope at La Silla (Chile) and the $0.5 \mathrm{~m}$ and $0.75 \mathrm{~m}$ telescopes at SAAO (South-Africa).

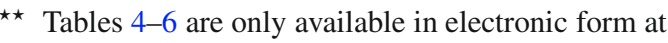
http: //www . aanda.org

$\star \star \star$ Postdoctoral fellow of the Fund for Scientific Research, Flanders.

$\dagger$ Ph.D. student of the Fund for Scientific Research, Flanders.

\section{Introduction}

Post-AGB stars are low- and intermediate-mass stars that evolve rapidly from the asymptotic giant branch (AGB) toward the planetary nebulae phase (PNe), before cooling to form a white dwarf. The processes that govern the transition between the symmetry in the AGB outflows and the observed large variety in geometries of proto-PNe and PNe, are still poorly understood (Balick \& Frank 2002; Sahai et al. 2007, and references therein). During the transition time, the star and its circumstellar envelope must 
be subject to fundamental and rapid changes in structure, massloss mode, and geometry. It is now generally acknowledged that binary interaction in these evolved stars could be responsible for a large fraction of the observed asymmetric nebular morphologies, but direct observational evidence of binarity in PNe is weak (Moe \& De Marco 2006; Zijlstra 2007). An analysis of the lightcurves obtained by the OGLE microlensing survey towards the Galactic bulge, inferred a close binary fraction of $12-21 \%$ (Miszalski et al. 2009). These data are only sensitive to the detection of systems which underwent a strong orbital decrease during interaction.

Testing the (wide) binary hypothesis is severely limited by the lack of observational information about binarity in PNe and the often very obscured proto planetary nebulae (PPNe), and also by our poor theoretical understanding of AGB evolution in binary systems. A major shortcoming is that detailed studies of individual, often spectacular, evolved objects prevail and systematic studies of a homogeneous sample are lacking. To study late stellar evolution in binary systems, optically bright, less obscured post-AGB stars are ideal candidates, and it has become clear that binarity is indeed very common (Van Winckel 2007).

The first binary post-AGB stars were serendipitously discovered and found to have distinct observational characteristics, including broad dust excesses often starting already at $\mathrm{H}$ or $\mathrm{K}$, inferring the presence of both hot and cool dust around the system. It was postulated that this indicates the presence of gravitationally-bound circumstellar material in the system (Van Winckel 2003, and references therein). The most famous example is the Red Rectangle, for which the Keplerian kinematics have been resolved by interferometric $\mathrm{CO}$ measurements (Bujarrabal et al. 2005).

These characteristics of their SEDs allowed us to develop a more systematic search for candidate binaries. In de Ruyter et al. (2006), we presented 51 objects and the number of known examples has since increased. The total sample is now a fair proportion of the 326 "very likely post-AGB stars" known in the Galaxy (Szczerba et al. 2007). Our selection criteria focused on infrared colours and resulted in including the RV Tauri stars with a dust excess detected by IRAS, the known binary post-AGB stars and newly characterised objects selected by Lloyd Evans (1999) on the basis of their position in the "RV Tauri" box in the IRAS [12]-[25], [25]-[60] two-colour diagram (see also Maas et al. 2003).

A typical spectral energy distribution is shown in Fig. 1. The stellar flux reprocessed into the infrared is about $50 \%$ for this star. The interested reader is referred to de Ruyter et al. (2006) for the SEDs of the complete sample. In all objects of the sample, the dust excess starts at, or very close to, dust sublimation temperature, irrespective of the spectral type of the central star. With a typical luminosity of a post-AGB star, this sublimation temperature edge is within a distance of $10 \mathrm{AU}$ from the central source. We therefore inferred (e.g. Van Winckel 2003) that part of the dust must be gravitationally bound: any typical AGB outflow velocity would bring the dust to cooler regions within years. This time is much shorter than the likely evolutionary timescale of the central object. We argued that the same inner geometry, found in the resolved system HD 44179 (Men'shchikov et al. 2002; Cohen et al. 2004; Bujarrabal et al. 2005), applies to the whole sample, i.e., the objects seem to be surrounded by Keplerian discs of dust.

Our interferometric studies confirm the very compact nature of the circumstellar material (Deroo et al. 2006, 2007). Our Spitzer and ground-based $N$-band spectroscopic data show that dust grain processing is strong, the oxygen-rich dust being dominated by, considerably larger, crystalline silicates (Gielen et al. 2008). Sub-mm bolometric data, which is available for only a few sources, show the presence of large grains in the circumstellar environment (De Ruyter et al. 2005). These large grains have relatively small dust-settling times, probably causing the disc to be inhomogeneous with small hot grains dominating the surface layers and a cooler midplane of mainly large grains (Gielen et al. 2007).

The true structure of those discs, in addition to their formation, stability, and evolution, are not well understood. We therefore initiated an extensive multiwavelength study of those systems. In this contribution, we report on our detailed radial velocity monitoring programme on the $1.2 \mathrm{~m}$ Swiss Euler telescope. The complementary photometric monitoring was performed at both SAAO and the $1.2 \mathrm{~m}$ Flemish Mercator telescope. The main aim of this programme was to investigate the link between binarity and the presence of gravitationally bound material, and gain insight into the final evolution of what appears to be a significant population of binary stars.

We organised the paper as follows. In the next section we introduce the sample stars as well as the specific criteria used to select the objects discussed in this paper. In Sect. 3, we sketch the data gathering procedure as well as the reduction methods. In Sect. 4, we focus on the radial velocity determination, and in Sect. 5, we report on the analyses of the sample stars individually. In Sect. 6, we discuss the binary frequency of the sample and the analyses of the orbital elements. We then place our results in a broader context of stellar evolution.

\section{Sample}

The total sample selected by our specific criteria is described in detail in de Ruyter et al. (2006).

In this paper, we restricted ourselves to the stars in our radial velocity programme with spectral type F, five in total, which exhibit only small amplitude photometric variability with a peakto-peak amplitude of up to $0.25 \mathrm{mag}$ in the $V$-band and are accessible from the southern hemisphere. In addition, we included HD 131356 (EN TrA), which was recognised to be of RV Tauri type by Pel (1976) and found to have a large excess in L by Lloyd Evans (1985), despite the higher peak-to-peak pulsational amplitude. The main reason for its inclusion is that, because of the longer time baseline of our velocity monitoring, we were able to confirm the binary nature of the star and update the period estimate of Van Winckel et al. (1999). It shares the early F spectral type of the other stars discussed here, and in the 2001/2 season, its $\mathrm{V}$ amplitude was only $0.3 \mathrm{mag}$.

The spectral types are taken from objective prism work (Houk et al. 1976; MacConnell \& Bidelman 1976; Kwok et al. $1997)$ in the case of IRAS 12222-4652 and EN TrA. The four remaining stars, which were all initially selected for their large infrared excesses (Lloyd Evans 1999), were classified by Lloyd Evans on the basis of blue light spectra of resolution $7 \AA$. Only the higher Balmer lines are relatively unaffected by emission. Intrinsic metal deficiency and subsequent depletion, weaken the metallic lines and vitiate the usual classification criteria. Luminosity class Ib was assumed to be broadly consistent with both the sharpness of the hydrogen lines and the similarity of the other properties of these stars to those of type II Cepheids. Published spectral types of B7III for IRAS 19125+0343 (Kwok et al. 1997) and B9Ib or B1III for IRAS 19157-0247 (Kwok et al. 1997; Parthasarathy et al. 2000; Gauba et al. 2003) may be discounted, since red spectra show strong lines of the CaII triplet, 
Table 1. General characteristics of the sample.

\begin{tabular}{|c|c|c|c|c|c|c|c|c|c|c|c|}
\hline$\overline{\text { IRAS }}$ & HD & sp. type & $\alpha_{2000}$ & $\overline{\delta 2000}$ & 1 & $\mathrm{~b}$ & $\mathrm{~m}(\mathrm{v})$ & $\bar{T} T_{\text {eff }}(\mathrm{K})$ & $\log g$ & $\overline{[\mathrm{Fe} / \mathrm{H}]}$ & $\overline{\text { Reference }}$ \\
\hline 08544-4431 & V390 Vel & F3 & 085614.18 & -444310.7 & 265.51 & +0.39 & 9.13 & 7250 & 1.5 & -0.5 & Maas et al. (2005) \\
\hline $12222-4652$ & $\begin{array}{l}\text { HD } 108015 \\
\text { NSV } 5601\end{array}$ & $\mathrm{~F} 3 \mathrm{Ib}$ & 122453.50 & -470907.5 & 298.25 & +15.48 & 7.95 & 7000 & 1.5 & -0.1 & Van Winckel (1997) \\
\hline $14524-6838$ & $\begin{array}{l}\text { HD } 131356 \\
\text { EN TrA }\end{array}$ & $\mathrm{F} 2 \mathrm{Ib}$ & 145700.69 & -685022.9 & 313.90 & -8.68 & 8.77 & 6000 & 1.0 & -0.7 & Van Winckel (1997) \\
\hline $15469-5311$ & & F3 & 155043.80 & -532043.3 & 327.82 & +0.63 & 10.56 & 7500 & 1.5 & 0.0 & Maas et al. (2005) \\
\hline $19125+0343$ & & $\mathrm{~F} 2$ & 191501.17 & +034842.7 & 39.02 & -3.49 & 10.16 & 7750 & 1.0 & -0.5 & Maas et al. (2005) \\
\hline $19157-0247$ & & F3 & 191822.71 & -024210.8 & 33.59 & -7.22 & 10.70 & 7750 & 1.0 & -0.4 & Maas et al. (2005) \\
\hline
\end{tabular}

Table 2. General characteristics of the spectral dataset obtained for every object.

\begin{tabular}{lrrcrc}
\hline \hline $\begin{array}{l}\text { Object } \\
\text { IRAS }\end{array}$ & $N_{\text {tot }}$ & $N_{\text {used }}$ & $\Delta$ HJD (24+) & $\begin{array}{r}\Delta V_{\text {rad }} \\
\mathrm{km} \mathrm{s}^{-1}\end{array}$ & $\Delta V$ \\
\hline $08544-4431$ & 181 & 161 & $51147-54487$ & 22.1 & 0.25 \\
$12222-4652$ & 83 & 82 & $45397-53833$ & 13.5 & 0.23 \\
$14524-6838$ & 75 & 63 & $48313-53904$ & 45.7 & 0.81 \\
$15469-5311$ & 175 & 161 & $51278-54680$ & 28.2 & 0.25 \\
$19125+0343$ & 103 & 90 & $51278-54331$ & 29.6 & 0.19 \\
$19157-0247$ & 137 & 111 & $51279-54680$ & 24.2 & 0.23 \\
\hline
\end{tabular}

which have an excited lower state and so do not appear as interstellar absorption lines, whereas the $\mathrm{H}$ and $\mathrm{K}$ lines of interstellar origin may be quite strong.

Cooler objects of the entire sample of de Ruyter et al. (2006) are in the Population II Cepheid instability strip and have photospheric pulsations of far larger amplitude. These stars have typical hydrogen line spectral types of G0 (Lloyd Evans 1999). In many of those, the pulsations show substantial cycle-to-cycle variability. These pulsations often show the defining alternating deep and shallow minima of RV Tauri stars. The detection of orbital motion in those objects is very difficult because of confusion with the radial velocity variations induced by the photospheric pulsations (Maas et al. 2002; Van Winckel et al. 1998, 1999).

In Table 1, the programme stars are listed with their spectral type, equatorial coordinates, Galactic coordinates, and visual mean magnitude. Mean photospheric characteristics are also given, including the appropriate reference.

\section{Data and data reduction}

In the framework of the cooperation between the Geneva Observatory and the Institute of Astronomy in Leuven (Belgium), the twin $1.2 \mathrm{~m}$ telescopes Euler (La Silla) and Mercator (La Palma) were constructed. Within this framework, the observers of Leuven have regular access to the Swiss Euler telescope operated by the Geneva observatory and the observers of the Geneva Observatory have regular access to the Flemish Mercator telescope operated by the Institute in Leuven. During the Leuven telescope time on Euler, typically 3 runs of 10-14 days every semester, the programme stars were monitored in radial velocity with the spectrograph CORALIE (Queloz et al. 1999). We started this campaign at the end of November 1998. In Table 2 we list the main characteristics of the observational data. $N_{\text {tot }}$ is the number of observations, $N_{\text {used }}$ the number used after our quality test (see later in the text), and $\triangle \mathrm{HJD}$ is the time range of the observations. The total peak-topeak radial velocity spread, $\Delta V_{\text {rad }}\left(\mathrm{km} \mathrm{s}^{-1}\right)$ and $\Delta V$, the visual photometric peak-to-peak variation, are also shown.

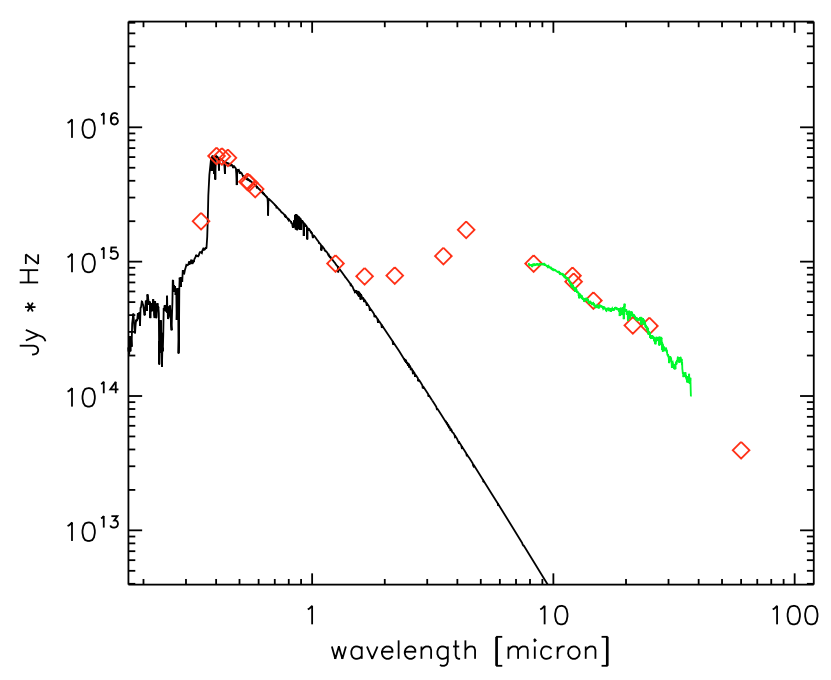

Fig. 1. The SED of IRAS $19125+0343$. The SED is typical for the sample, showing a large and broad IR-excess. A total reddening of $E(B-V)=1.1$ was deduced by minimizing the difference between the dereddened broadband fluxes (diamonds) and the appropriate model atmosphere (full black line). The infrared excess was not considered in this minimalisation. The Spitzer infrared spectrum (Gielen et al. 2008) is also shown.

The CORALIE spectrograph is a fibre-fed spectrograph that samples the visual spectrum in 68 spectral orders ranging from $388 \mathrm{~nm}$ to $682 \mathrm{~nm}$. The resolving power $(\lambda / \Delta \lambda)$ is 50000 at $500 \mathrm{~nm}$, with a 3 pixel sampling. The fibre diameter is 2 arcsec on the sky. We used the online data reduction system (Queloz et al. 1999), which includes all standard steps in echelle reduction.

Older radial velocity data of IRAS 12222-4652 and EN TrA are also included. These data were obtained with the CES spectrograph mounted on the CAT telescope at La Silla (ESO) as well as with the CORAVEL instrument mounted on the $1.5 \mathrm{~m}$ Danish telescope also on La Silla. CORAVEL is a spectrophotometer with which the radial velocity was obtained by crosscorrelation of the stellar spectrum with a hardware mask created from the spectrum of the K2 III star Arcturus (Baranne et al. 1979). EN TrA has stronger lines than the other stars observed, so that clear correlation profiles are obtained from these data. The zeropoint of the system was regularly assessed during the night by measuring CORAVEL radial velocity standards. These calibrations were performed at intervals of about $1-2 \mathrm{~h}$. All the CORAVEL observations, including ours, are held in a database that is maintained by the owners of the CORAVELs in Geneva.

The stars accessible from La Palma were included in the long-term photometric monitoring programme performed with the $1.2 \mathrm{~m}$ Mercator telescope using the refurbished Geneva 7-band dual-beam photometer (Raskin et al. 2004). This 
Table 3. General characteristics of the photometric datasets obtained for every object.

\begin{tabular}{lccc}
\hline \hline Object & P7 & SAAO & ASAS \\
\hline IRAS 08544-4431 & & $91(50858-52060)$ & $830(51868-54843)$ \\
IRAS 12222-4652 & $120(47258-47824)$ & $166(51625-53195)$ & $576(52441-54711)$ \\
EN TrA & $61(47608-49853)$ & $58(43979-49564)$ & $633(51905-54743)$ \\
IRAS 15469-5311 & & $49(48803-51712)$ & $550(51925-54743)$ \\
IRAS 19125+0343 & $47(52415-53606)$ & $30(49485-51819)$ & $362(52442-54772)$ \\
IRAS 19157-0247 & $6(52446-52561)$ & $50(48404-51819)$ & $386(51979-54777)$ \\
\hline
\end{tabular}

resulted in 47 high quality absolute photometric measurements for IRAS 19125+0343, but only 6 for IRAS 19157-0247. The seven bands are measured quasi-simultaneously using a filter wheel that cycles through all seven filters four times per second alternating as well between the sky and the object channel (Golay 1980). The same instrument was previously attached to the now decommissioned $0.7 \mathrm{~m}$ Swiss telescope at La Silla. During that period, we obtained many measurements for the Southern stars, IRAS 12222-4652 and EN TrA.

The photometric observations in the Cousins UBVRI system were obtained from SAAO with the Modular Photometer on the $0.5 \mathrm{~m}$ telescope and subsequently with a very similar photometer on the $0.75 \mathrm{~m}$ Automatic Photoelectric Telescope (Martinez et al. 2002). These observations were made with reference to the Cousins standards in the E-regions (Menzies et al. 1989). The data are contained in Table 5, except for EN TrA for which the observations were made by Berdnikov \& Turner (1995) and Caldwell et al. (2001).

Since these monitoring programmes require the dedicated efforts of many people, the observers responsible for the data acquisition for this project are included as co-authors in alphabetical order.

We complemented our own observations with the $V$-band data available from the All Sky Automated Survey (Pojmanski 2002). These data were obtained with the ASAS-3 configuration using the wide field $\left(8.8^{\circ} \times 8.8^{\circ}\right)$ CCD camera with a sampling of $\sim 15^{\prime \prime} /$ pix. The critical PSF sampling is not reached and the pipeline reduction approximates aperture photometry with 5 circular apertures from 2 to 6 pixels in diameter. The ASAS guidelines foresee a preferred aperture for every magnitude bin and we limited our analysis to measurements with quality flag A, according to the less rigorous classification applied to the rereduced data on the ASAS website. The recommended apertures were used, except that for IRAS 19125+0343 we used aperture 4, MAG-3, rather than aperture 1, MAG-2, to ensure full inclusion of the $\mathrm{M}$ star at an angular distance of 9.9 arcsec. We applied corrections for the small offsets between the magnitudes of the same star observed with different cameras and for the offsets between the original and later reductions of early observations, many of which were not included in the re-reduced data on the ASAS website after recovery from the data storage crash. The photometric zeropoint is tied to the Hipparcos catalogue, since in every frame a few hundred Hipparcos stars are located. These were used in the zeropoint definition (Pojmanski 2002). Near simultaneity of some of our observations with ASAS data enabled determination of zeropoint differences and error estimates. The offsets (ASAS-other), standard deviation (s.d.) of the single observations, the number of data points, and the source of comparison data are: IRAS 085444431, +0.043 mag, $0.010 \mathrm{mag}, 14$, SAAO; IRAS 12222-4652, $+0.009,0.015,44$, SAAO; IRAS 19125+0343, -0.057, 0.022, 18, P7; IRAS 19157-0247, +0.043, 0.023, 3, P7. These differences have been allowed for in combining the relevant datasets.
Additionally, from non-overlapping datasets, we have offsets for IRAS 15469-5311, -0.030, SAAO; IRAS 19125+0343, +0.045, SAAO; IRAS 19157-0247, +0.070, SAAO. The SAAO magnitudes of IRAS $19125+0343$ are 0.09 mag brighter than the P7 observations, because of the inclusion of the $\mathrm{M}$ star in the aperture used at SAAO. These last values will include any true changes in brightness between the two epochs.

The shift between SAAO and P7 for IRAS 19125+0343 is attributable to the inclusion or exclusion of the nearby $M$ type star. The reason for offsets for other stars is unknown. The s.d. obtained by direct comparison of photometric data, are smaller than the errors ascribed to individual observations in ASAS, which must therefore be more precise than previously recognised. Intercomparison of three independent subsets within the ASAS data for IRAS 08544-4431 gives values in the range 0.011 to 0.024 mag, confirming the typical values above.

The ASAS data proved to be valuable in compensating for the poorer sampling of our own multi-colour measurements in the search for the long periods expected. ASAS data were used by Kiss et al. (2007) in their study of the pulsations of these and similar stars, but the continuation of the ASAS project has doubled the timespan of the observations, which now extend from 2001 or 2002 to the end of the 2008 season. Moreover, the rereduction of much lost ASAS data has filled the gap around JD 2453500 seen in the plots by Kiss et al. (2007) for these stars. The more extensive data facilitate the characterisation of the noisy small amplitude oscillations of these stars.

In Table 3, we list the main characteristics of the raw photometric data sets for all objects. The number of measurements in the different photometric systems is given. P7 is the 7-band Geneva photometric system, SAAO is the Cousins UBVRIsystem, and the ASAS monitoring is obtained in the $V$-band only. In brackets we show the total time coverage in HJD (24+).

\section{Radial velocity determination}

To obtain accurate radial velocity information based on low $\mathrm{S} / \mathrm{N}$ spectra, we determined cross-correlation profiles for all individual measurements. The standard spectral mask includes a large set of distinctive spectral lines for the given spectral type. Our chemical analysis, based on high signal-to-noise optical spectra, showed that for most stars, several elements, including iron, are underabundant (Maas et al. 2005). By eliminating, from the standard spectral mask, those lines not detected in our highquality spectrum, we created an appropriate spectral mask for every individual star. Moreover, we selected only single lines with clear symmetric profiles.

In Fig. 2, we compare the cross-correlation profile obtained by the standard mask with the one obtained by the specific mask, constructed on the basis of the high signal-to-noise ratio spectrum for IRAS 19157-0247. No accurate velocity can be derived by using the standard mask, while a Gaussian fit of the profile, 


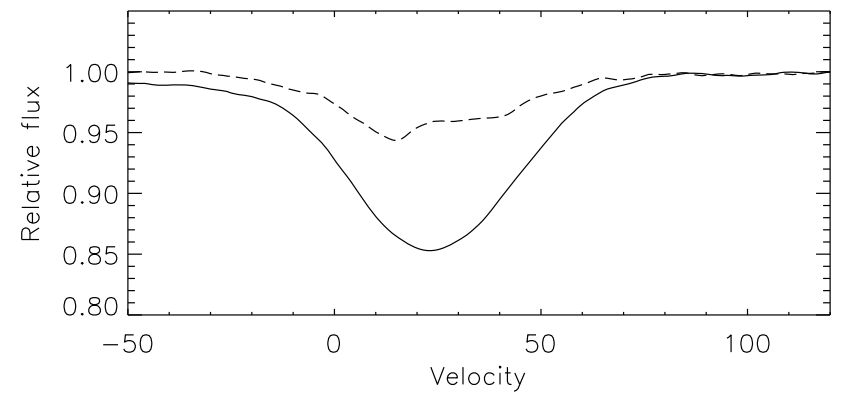

Fig. 2. Comparison between cross-correlation profiles obtained with a standard spectral mask (dashed line) and with a software mask adapted to the individual spectral characteristics of IRAS 19157-0247 (full line).

obtained by the specified mask for IRAS 19157-0247, provides a good estimate of the radial velocity.

The radial velocity is computed using the mean of a 50-point bisector of the cross-correlation function. An initial Gaussian fit was used to determine the bisector points, which are defined on an equidistant velocity grid centred on the velocity minimum of the Gaussian fit. The grid spans twice the Gaussian width $(\sigma)$. The internal uncertainty in a radial velocity measurement is estimated from the rms of these 50 bisector values. We eliminated these cross-correlation profiles for which the rms is larger than $1 \mathrm{~km} \mathrm{~s}^{-1}$. Several spectra were obtained in very poor weather conditions and/or guiding failures. These measurements were eliminated in this step of the analysis. Although the objects do not have a large pulsational amplitude, some objects do show asymmetries in these cross-correlation profiles at particular phases in the pulsation cycle. These profiles are also eliminated at this point bacause our study focuses on the detection of orbital motion. The number of radial velocity measurements that passed our critical quality assessment test is given in Table 2 . The individual datapoints are given in Table 4.

\section{Variability study of individual stars}

The main goal of our programme was to search for evidence of binary motion in the radial velocity data and, if found, determine the orbital elements. Although for this paper, we selected objects with a small pulsational amplitude in $\mathrm{V}$, they all show some photometric variability, which could also be present in the radial velocity data.

Luminous stars on the blue side of the population II Cepheid instability strip are, however, far from being regular monoperiodic pulsators and often exhibit a very complex pulsational behaviour. One example is HD 56126 (Barthès et al. 2000; Fokin et al. 2001), where the main pulsation mode is shown to generate shocks provoking a complex asynchronous motion in the outer layers. Another example is HR 7671 (HD 190390), where the main period of 28.6 days is accompanied by a beating behaviour caused either by a stable pulsation triplet or an unstable main period. Consequently, the description of the pulsation in radial velocity, modelled as a simple harmonic, fails to reproduce the complete dataset, which has a peak-to-peak difference of $8.2 \mathrm{~km} \mathrm{~s}^{-1}$ (Reyniers \& Cuypers 2005), as well as a corresponding light amplitude of $0.36 \mathrm{mag}$. Neither object shows evidence of binary motion. Since the programme stars of this project are also on the hot side of the instability strip, we investigated the photometric dataset in parallel with the radial velocity data to compare radial velocity variability with photometric behaviour.

For the variability study, we used phase dispersion minimization PDM (Stellingwerf 1978) and, for the photometric data,

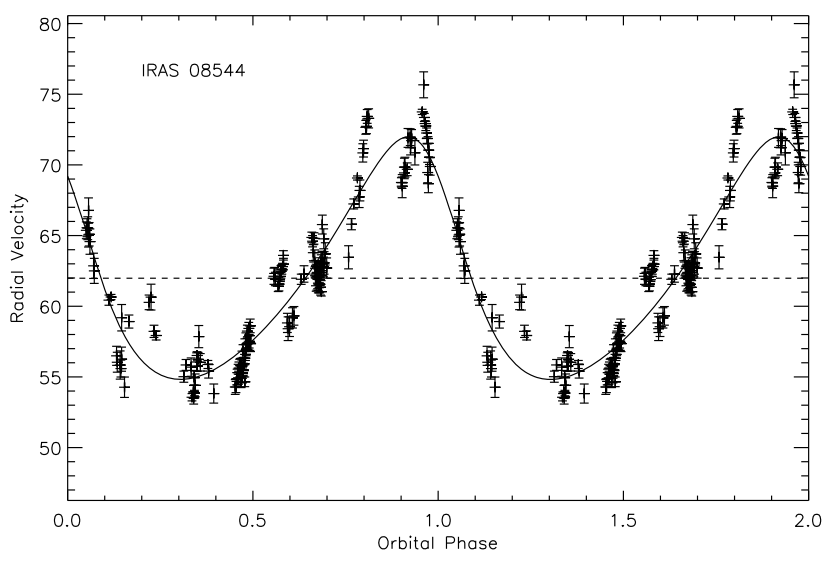

Fig. 3. The radial velocities folded on the orbital period of 508 days for IRAS 08544-4431. The errorbars are defined as the standard deviation in the 50-point bisector of the cross-correlation profile. The system velocity is given by a dashed line.

the Lomb-Scargle method to search for periodicities. The orbital solutions were then computed using an altered version of the Fortran code VCURVE (Bertiau \& Grobben 1969) as well as the minimisation code FOTEL (Hadrava 2004). The weights of the individual velocity points were based on the rms values of the bisector analyses. All errors quoted in the individual orbital elements are rms errors of these elements calculated from the covariance matrix. Plots of the photometry of single seasons were used to look at the individual cycles in some cases. The individual photometric datapoints are given in Tables 5 and 6.

\subsection{IRAS 08544-4431}

We demonstrated that this object is a spectroscopic binary in Maas et al. (2003). We continued our monitoring of this object to refine its orbital elements. We gathered a total of 161 good radial velocity measurements sampling more than 6 orbital periods. The resulting radial velocity variations are shown in Fig. 3. An orbital period of 508 days and an eccentricity of 0.25 were found. All orbital elements are listed in Table 7. In this table we list $P$ as the orbital period in days, $K$ as the velocity amplitude, $e$ as the eccentricity, $\omega$ as the angle in the orbital plane between the direction to the ascending node and the direction to the periastron, $T_{0}$ as the time of periastron passage, $\gamma$ as the system velocity, rms $(\mathrm{O}-\mathrm{C})$ as the root mean square of the errors, $a_{1} \sin i$ as the semi major axis, $F(M)$ as the mass function in solar masses, $N$ as the number of observations, $\Delta t$ (cycles) as the number of cycles used and $\Delta V$ as the visual photometric peak-to-peak variation.

Our previous pulsational analysis of photometry from SAAO (Maas et al. 2003) showed that low-amplitude pulsations are present, but that the cycle-to-cycle variability is large. Periods of 72.3 and 89.9 d were reported. Kiss et al. (2007) confirmed the shorter period and obtained two new periods, $68.9 \mathrm{~d}$ and 133 d, from ASAS data that extended to JD 2453900 , with a break $2453200-2453750$.

We combined the SAAO and the highest quality ASAS dataset (see Table 3) and analysed the total $V$-band time-series. In Fig. 4, the total dataset is shown and a beating pattern with a clear amplitude modulation is present, as well as a slow brightening. The dominant period of $71.7 \pm 0.4 \mathrm{~d}$ gives in a harmonic fit of 0.03 mag amplitude and a variance reduction of $21 \%$. 
Table 7. The orbital elements of the programme stars.

\begin{tabular}{|c|c|c|c|c|c|c|c|c|c|c|c|c|}
\hline & \multicolumn{2}{|c|}{$\overline{\text { IRAS } 08544}$} & \multicolumn{2}{|c|}{ IRAS 12222} & \multicolumn{2}{|c|}{ ENTra } & \multicolumn{2}{|c|}{ IRAS 15469} & \multicolumn{2}{|c|}{ IRAS 19125} & \multicolumn{2}{|c|}{ IRAS 19157} \\
\hline & & $\sigma$ & & $\sigma$ & & $\sigma$ & & $\sigma$ & & $\sigma$ & & $\sigma$ \\
\hline Period (days) & 507.8 & 1.5 & 913.8 & 4.3 & 1493. & 7. & 389.9 & 0.5 & 519.6 & 2 & 119.5 & 0.2 \\
\hline$K\left(\mathrm{~km} \mathrm{~s}^{-1}\right)$ & 8.6 & 0.3 & 3.5 & 0.5 & 16.3 & 0.6 & 11.9 & 0.3 & 12.6 & 0.5 & 7.3 & 0.7 \\
\hline$e$ & 0.24 & 0.02 & 0.00 & 0.0 & 0.32 & 0.04 & 0.09 & 0.02 & 0.25 & 0.03 & 0.31 & 0.07 \\
\hline$\omega\left(^{\circ}\right)$ & 47. & 7. & & & 160. & 6. & 133. & 16. & 242. & 6. & 80. & 14. \\
\hline$T_{0}$ (periastron) (JD24+) & 51482. & 10. & & & 538535. & 22. & 51491. & 17 & 53061. & 8. & 51849. & 5. \\
\hline$\gamma\left(\mathrm{km} \mathrm{s}^{-1}\right)$ & 61.98 & 0.16 & 3.65 & 0.17 & -7.1 & 0.4 & -14.9 & 0.2 & 67.0 & 0.3 & 33.1 & 0.3 \\
\hline $\mathrm{rms}\left(\mathrm{km} \mathrm{s}^{-1}\right)$ & 1.88 & & 1.47 & & 2.42 & & 2.1 & & 1.98 & & 3.31 & \\
\hline$a_{1} \sin i(\mathrm{AU})$ & 0.39 & 0.01 & 0.29 & 0.04 & 2.12 & 0.08 & 0.42 & 0.01 & 0.58 & 0.02 & 0.08 & 0.01 \\
\hline$F(M)\left(M_{\odot}\right)$ & 0.0304 & 0.0033 & 0.0042 & 0.0017 & 0.57 & 0.06 & 0.068 & 0.006 & 0.097 & 0.012 & 0.0041 & 0.0012 \\
\hline$N$ & 161 & & 82 & & 63 & & 161 & & 90 & & 111 & \\
\hline$\Delta t$ (cycles) & 6.6 & & 9.2 & & 3.8 & & 8.7 & & 5.9 & & 28.4 & \\
\hline$\Delta m(V)$ & 0.25 & & 0.23 & & 0.81 & & 0.25 & & 0.19 & & 0.23 & \\
\hline
\end{tabular}

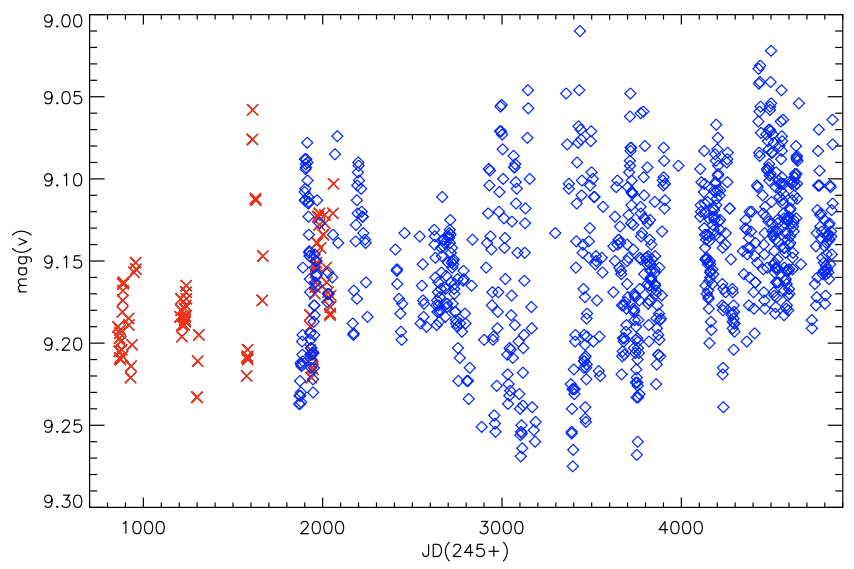

Fig. 4. The combined datasets of the $V$-band photometric measurements of IRAS 08544-4431. The $x$-symbols are the SAAO data and the diamonds are the ASAS measurements.

The amplitude was greatest, at $0.20 \mathrm{mag}$, during the interval from JD 2452850 to 2453500 . Figure 5 shows these data, phased at the double period $143.6 \mathrm{~d}$. The alternate minima are of different depth and the alternate maxima are of unequal height, although the differences are quite small. The behaviour was maintained for almost 2 years and is reminiscent of the typical RV Tauri light curves, albeit of a far smaller amplitude. Much of the small scatter may be attributed to the observational error of about $0.02 \mathrm{mag}$. Corresponding plots for the two intervals of small amplitude, represented by SAAO data from JD 2450850 to 2451500 and ASAS data from JD 2452400 to 2452750 show amplitudes of $0.08 \mathrm{mag}$ and $0.06 \mathrm{mag}$, respectively, while the curves are less clearly defined. The separation of these intervals, at least $1400 \mathrm{~d}$, is less than expected for a beat between the two principal periods, 2800 d, as suggested by Kiss et al. (2007), although the next interval of small amplitude had not occurred by early 2009, JD 2454 840, and may yet fit the prediction.

There is evidence of this orbital period in neither the ASAS photometry (Kiss et al. 2007) nor our extended multicolour photometry. Given the complex atmospheric pulsations, we did not attempt to clean the low amplitude irregular pulsational variability from the radial velocity dataset.

\subsection{IRAS 12222-4652}

IRAS 12222-4652 also exhibits a complex pulsational pattern with a significant amplitude modulation and a smaller amplitude

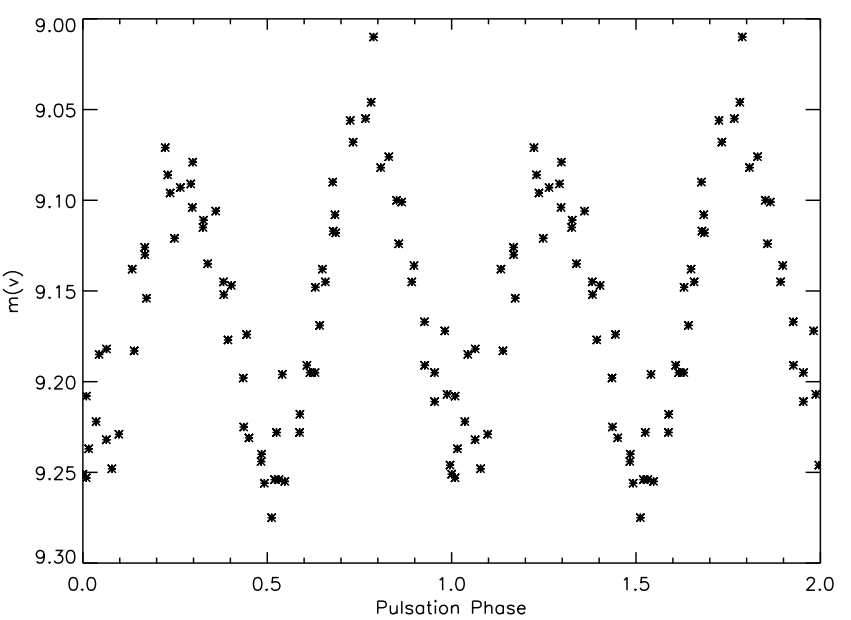

Fig. 5. The $V$ magnitudes for IRAS $08544-4431$ plotted against the double period, 143.6 days. Data of period JD 2452 887-2 453 397, when IRAS 08544-4431 showed a large amplitude.

at earlier times (see Fig. 6). ASAS data for the period before JD 2452300 have been rejected as they were highly erratic, in contrast to contemporaneous SAAO data. Our Geneva photometry was poorly sampled over many years, while the SAAO and ASAS photometry combine well to provide a larger dataset. We find a dominant period of $60.5 \pm 0.11 \mathrm{~d}$ and a second period of $55.3 \pm 0.2 \mathrm{~d}$, in agreement with (Kiss et al. 2007). A combined harmonic fit infers a fractional variance reduction of 52\%. Plots of the $V$ magnitude against time show continuous variability in the amplitudes and lengths of successive cycles, from 0.11 to 0.20 mag and from 48 to 76 days. The longer cycles tend to have amplitudes that are below average. The PDM analysis also yields an additional period of $120.6 \pm 1.0 \mathrm{~d}$, or twice the principal period. IRAS $12222-4652$ is clearly another star with a complex dynamical photosphere, which complicates the interpretation of its radial velocity variations.

The total radial velocity dataset of IRAS 12222-4652 includes older data, measured on high-quality spectra obtained to study the chemical composition (Van Winckel 1997). Our total timespan is therefore longer than 23 years. The dataset infers a significant initial period of $917 \pm 4$ days, which we interpret as due to orbital motion. A circular orbit gives a fractional variance reduction of $65 \%$. After cleaning this first circular model, we retrieve the dominant photometric period of $60.5 \mathrm{~d}$ in our $\theta$-statistic with an amplitude of $1.4 \mathrm{~km} \mathrm{~s}^{-1}$. Our final orbital solution (see Fig. 7) is then obtained after cleaning the original dataset with 


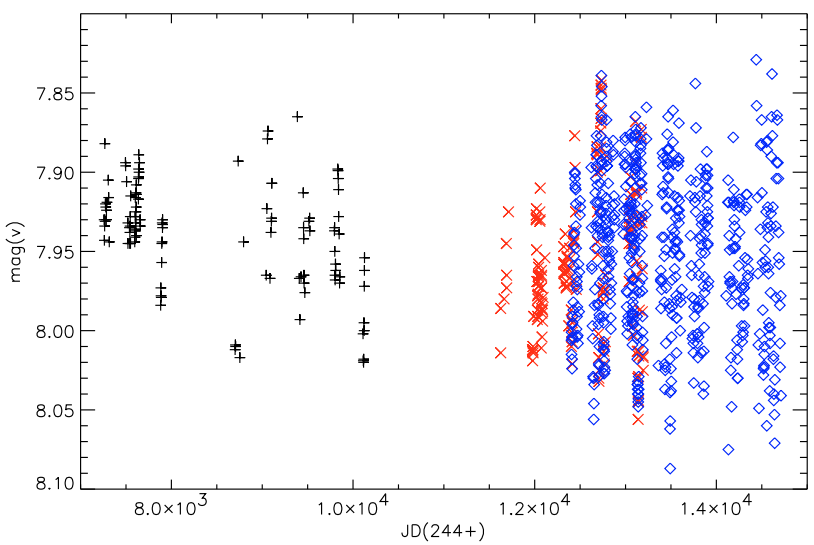

Fig. 6. The combined datasets of the $V$-band photometric measurements of IRAS 12222-4652. The + are the Geneva measurements, the $x$-symbols are the SAAO data and the diamonds are the ASAS measurements.

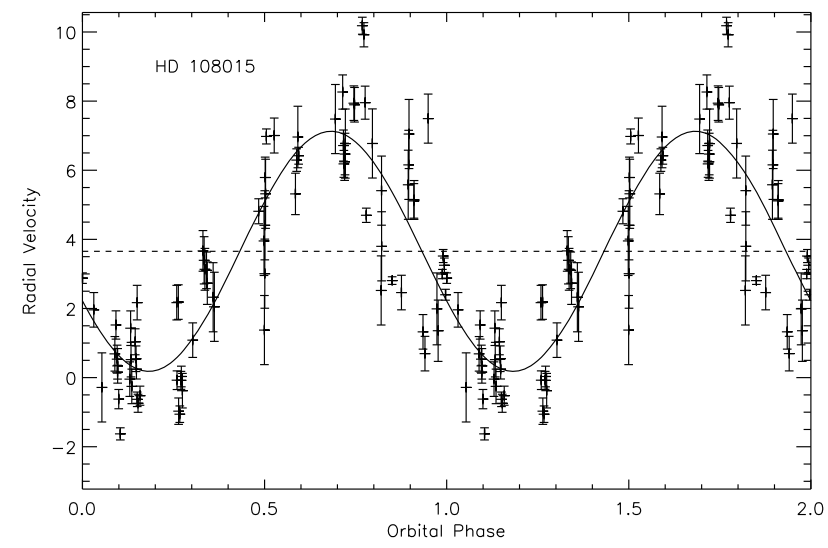

Fig. 7. The radial velocity variations of IRAS 12222-4652, after correction for the variations induced by the pulsations. The data are folded at the orbital period of 914 days. The full line is a circular model for the orbit.

the harmonic fit of the dominant photospheric pulsation mode. The small eccentricity does not differ significantly from zero, so our final solution gives a circular orbit of $914 \pm 4$ days. The fractional variance reduction is $75 \%$. The orbital period is not present, either in magnitude or colours.

\subsection{EN TrA}

HD 131356 (EN TrA) was discovered to be variable by Miss Leavitt (Pickering 1907). The binary nature and preliminary orbital elements were given in Van Winckel et al. (1999). We have since accumulated more data using the CORALIE spectrograph to improve the orbital solution.

The object is catalogued in the GCVS as a small amplitude Cepheid with a published pulsation period of $36.54 \mathrm{~d}$ (Grayzeck 1978), based on a limited dataset covering part of two pulsational cycles, with poor coverage in phase. Pel (1976) obtained a continuous time-series of 44 measurements on the Walraven photometric system. The object showed at that time (JD 2440 990-2441 171) a large $V$-band amplitude with a maximum of $V=8.55$, a deep minimum of 9.2, and a shallow minimum of 8.8. The Hipparcos catalogue (HIP 73152) lists a period of 37.10 days (Perryman \& ESA 1997).

Our Geneva photometry is poorly sampled and no periodicity could be determined. Two sets of observations obtained at

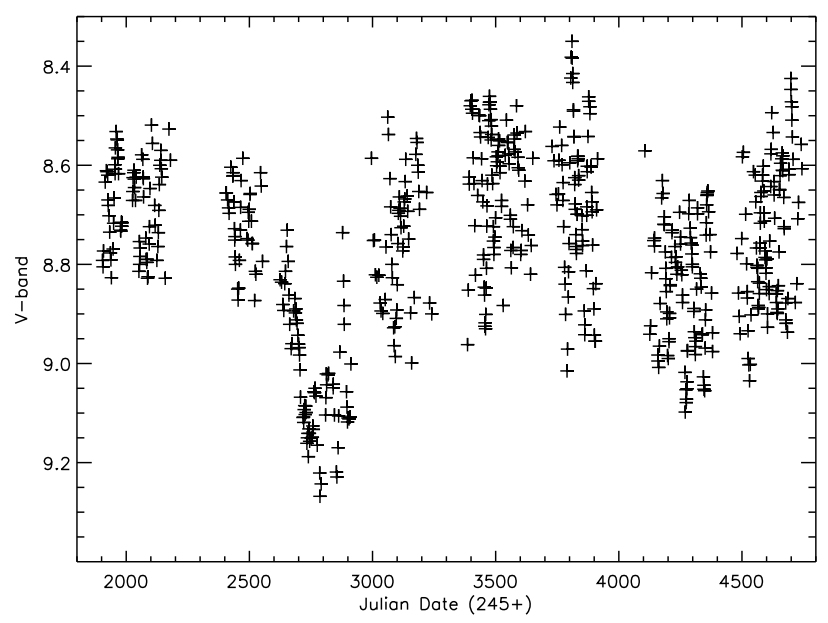

Fig. 8. The $V$-band photometric dataset of EN TrA obtained by the ASAS project (Pojmanski 2002).

SAAO on the UBVRI system are available, comprising 42 observations (JD 2443 979-2445 155) by Caldwell et al. (2001) and 16 observations (JD $2449522-2449564$ ) by Berdnikov \& Turner (1995). Both indicate a range of $V=8.5-9.05$, with little difference between deep and shallow minima. A short dataset by Eggen (1986) gives a range of $V$-magnitudes between 8.55 and 8.96, without full coverage of the extrema. The first season of ASAS data, 2001-2002, shows a variable amplitude of up to $0.3 \mathrm{mag}$, similar to the other stars (see Fig. 10).

EN TrA fades by some 0.5 mag near JD 2452800 and again, by about $0.1 \mathrm{mag}$, near JD 2454300 (see Fig. 8). Outside the intervals, the mean magnitude is fairly constant but the amplitude is variable. A period analysis infers a single period $P=37.04 \pm$ $0.03 \mathrm{~d}$ and a double period $74.1 \pm 0.1 \mathrm{~d}$. Aliases correspond to the orbital period and the calendar year. The magnitudes at maximum and minimum light are both very variable from cycle to cycle and from year to year. The deep and shallow minima, and bright and faint maxima, generally retain the same relative phasing within a given season, but they sometimes interchange in successive years. These phenomena are well known in RV Tauri stars.

Because of the very long baseline of our radial velocity data, we could sample about 3.7 orbital cycles. In a first iteration, after the removal of the orbital solution, the residuals showed a very clear periodicity with a period of $36.70 \mathrm{~d}$, which we link to the pulsation timescale found in the photometry. A harmonic fit inferred a radial velocity amplitude of $4 \mathrm{~km} \mathrm{~s}^{-1}$ and we cleaned the original data with this mean pulsation model. The final solution gives an orbit with a period of $1493 \pm 10$ days and a large eccentricity of $0.31 \pm 0.05$. (see Fig. 9, Table 7).

To investigate the secular variations in the mean magnitude, we selected only those pulsation cycles for which 6 or more data points are available. We then cleaned the pulsation amplitude and reduced the pulsation cycle to the mean magnitude alone. This mean magnitude is around 8.6, but there is a significant drop when the object enters the conjunction phase (see Fig. 9). The minimum (mean magnitude of 9.1) is obtained at exactly inferior conjunction and EN TrA then slowly recovers to its nominal mean values of about 0.3 in orbital phase later. There are shallower fadings at the same orbital phase: three orbits earlier, near JD 2448350 (P7 data) and JD 2454300 (ASAS data). The corresponding orbital phase is not covered by any of the other datasets. The most natural explanation of the secular variability is that the star experiences from variable circumstellar reddening 


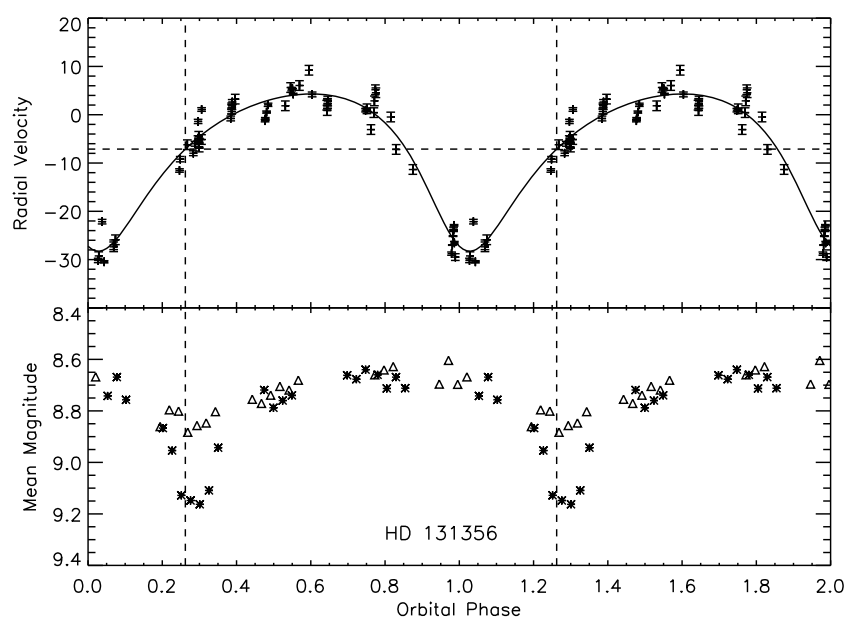

Fig. 9. The top panel shows the radial velocity variations of EN TrA folded at the orbital period of 1493 days. The full line represents the orbital model. The lower panel gives the mean magnitude determined on those pulsation cycles with more than six data points. The different symbols represent two different orbital cycles. Mean minimal light is measured when the object is at inferior conjunction but in the two different orbital cycles, the effect is not equally prominent.

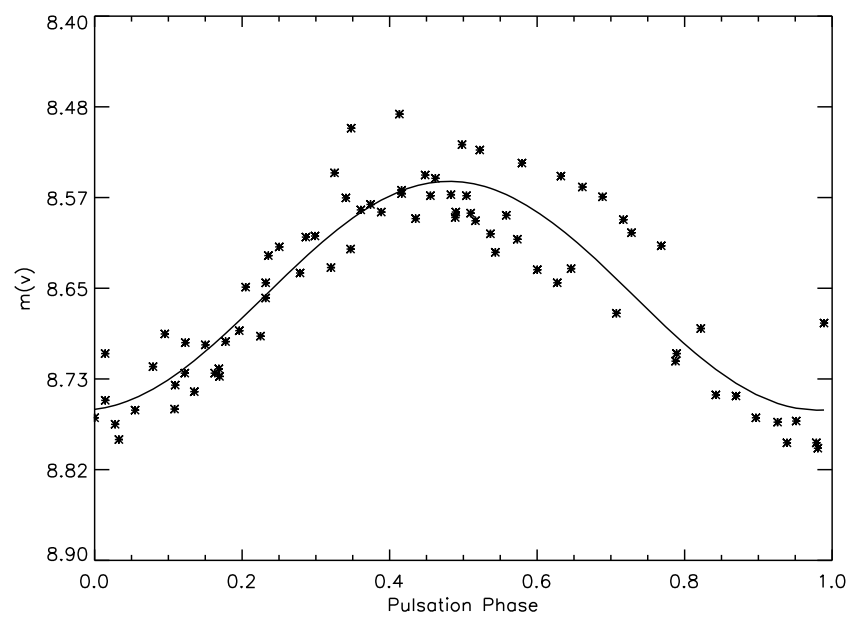

Fig. 10. The $V$-band magnitudes of EN TrA, from the ASAS photometric monitoring project of the first season (JD 2451 903-2 452 173), folded on the pulsation period of 36.70 days found in the radial velocity data.

during orbital motion. The minimum is reached when the object suffers from a maximal reddening of circumstellar material in the line-of-sight, which would occur when the star is at inferior conjunction if there is a circumbinary disk.

\subsection{IRAS 15469-5311}

IRAS 15469-5311 is another low-amplitude pulsator that has been little studied in the literature. The mean magnitude varies with a fairly abrupt brightening of 0.045 mag after JD 2454000 , and exhibits smaller variations on a timescale greater than 1000 days. We combined the ASAS and SAAO photometric $V$-band measurements for the pulsation study. The main period is $54.5 \pm 0.3 \mathrm{~d}$, which is consistent with that of $54.4 \pm 1 \mathrm{~d}$ found by Kiss et al. (2007), and a harmonic fit gives a fractional variance reduction of $21 \%$ with an amplitude of only 0.028 mag. The extended dataset did not confirm the second pulsation period of 49.1 days given by Kiss et al. (2007); this might be a one

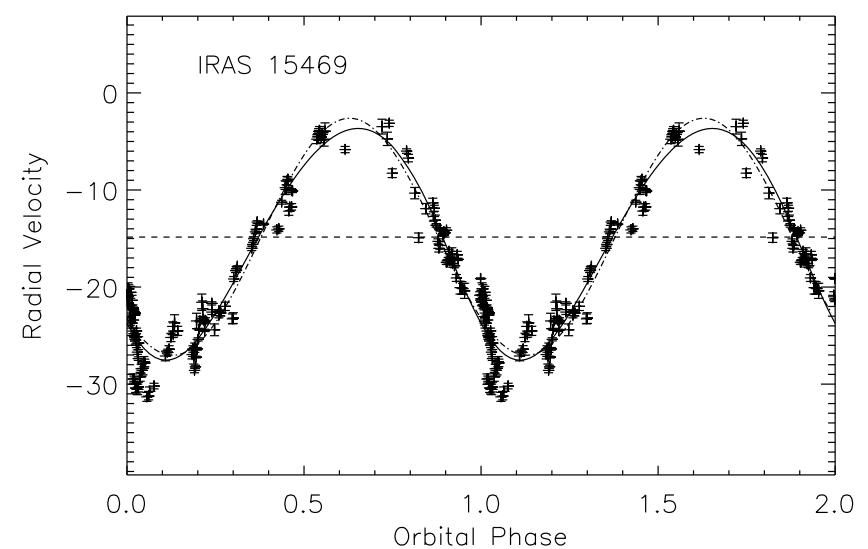

Fig. 11. The radial velocities of IRAS 15469-5311 folded ot the orbital period of 389.9 days. The full line is the orbital solution, and the dashdotted line is the best-fit circular orbital curve.

year alias of the primary period. The cleaned data yield the next possible period of $56.6 \pm 0.3 \mathrm{~d}$, but the variance reduction is limited. Possible other periods of 164 and 77 days may be spurious as well, as they also appear in the windowed data. Plots of magnitude against JD for 20 well observed cycles in four seasons give lengths of 32 to 77 days, with a mean of 54.0 days. The amplitude varies from $0.02 \mathrm{mag}$ to $0.15 \mathrm{mag}$. The mean amplitude quoted above is an underestimate of the true variability, as the mean light curve is blurred by variability in both amplitude and length of period. The double period also emerges from the PDM analysis and a plot of $V$ against 109.2 days shows a weak tendency towards alternate deep and shallow minima, as well as bright and faint maxima.

We have 160 good radial velocity measurements for IRAS 15469-5311 over a time range of 3402 days. The orbit of 390 days is very well defined (see Fig. 11). The classical Lucy and Sweeney test (Lucy \& Sweeney 1971) shows that the orbit has a small but significant eccentricity of 0.09. In Table 7, all other orbital parameters are given. There is an indication of the orbital period in the photometric data, for which Kiss et al. (2007) give a period of $384 \pm 50$ days, but the phase diagram is not convincing. The residual velocity variations have a peak-topeak amplitude of $10 \mathrm{~km} \mathrm{~s}^{-1}$ (standard deviation of $2.1 \mathrm{~km} \mathrm{~s}^{-1}$ ), but there is no clear indication of the main pulsation period nor could we discover a clear periodicity in the residuals.

\subsection{IRAS $19125+0343$}

IRAS $19125+0343$ is also a poorly studied object, despite having both rather bright IRAS fluxes and a significant visual flux of $m(v)=10.16$. The LRS-IRAS spectrum is featureless (Kwok et al. 1997) and the source was not detected in the OH survey of Le Squeren et al. (1992). The object was rediscovered by Lloyd Evans (1999) in the search for new RV Tauri stars.

The photometric variability is small: the SAAO and P7 datasets give ranges of 0.11 and $0.07 \mathrm{mag}$, respectively, in the $V$-band. Kiss et al. (2007) found a possible long period of $2300 \mathrm{~d}$; the more extensive ASAS data available now are consistent with this, with an amplitude of about $0.03 \mathrm{mag}$, but instead a step change of 0.03 mag fainter after JD 2453400 is a possibility.

Application of the period search algorithms to the complete dataset yielded several periods between 34 and 43 days, 42.3 and 38.9 days being the strongest. Phase plots against any of these periods showed much greater scatter than can be attributed 


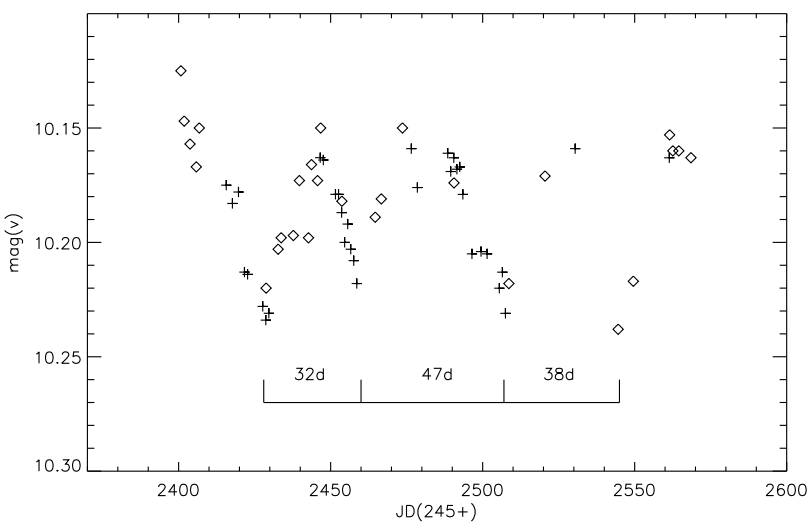

Fig. 12. Detail of the lightcurve of IRAS $19125+0343$. Period changes occur on irregular time intervals illustrating the strong cycle-to-cycle differences observed in this object. P7 data are denoted by plus signs and ASAS data by diamonds.

to observational errors. The light curves (Fig. 12) for individual seasons were inspected, and the times between identifiable features, generally near the mean magnitude on the ascending or declining branches of the light curve, were estimated for successive cycles. There were insufficient data in 2006, and variations were small and/or erratic in 2004, 2005, and 2007. Estimates of the period could be made for 1994, from SAAO data, for 2002, from P7 and ASAS data, and for 2003 and 2008 from ASAS data. The values for 15 cycles ranged from 29 to 51 days, with a mean of 38.2 days. On two occasions, an incipient decline after maximum was reversed to give a second maximum and an unusually long interval between mid-decline points. This occurred in 2002, turning a tentative 32 day interval into 47 days, and in 2003 , turning a 39 day interval into 51 days. The 519 d orbital period is not present in the photometry.

We have 91 good radial velocity measurements over a time range of 3389 days. We found a clear periodicity of 519 days and the peak-to-peak velocity amplitude is no less than $29.5 \mathrm{~km} \mathrm{~s}^{-1}$. Given the period and amplitude, we interpret this variability as being due to orbital motion. We obtained orbital elements with a significant eccentricity of $0.26 \pm 0.03$. We sampled in total 6.5 orbital cycles. Figure 13 shows the radial velocities folded on this period. The full line represents the best-fit Keplerian orbital solution (see Table 7).

The strongest peak in both the PDM and Scargle periodograms for the residual velocity variations corresponds to 43 days, which leads to a variance reduction of $39 \%$, and is fairly close to the mean of the cycle lengths found in the photometry. The orbital period is much shorter than the possible long photometric period (Kiss et al. 2007) in the ASAS data.

\subsection{IRAS 19157-0247}

The SAAO and ASAS photometry show that IRAS 19157-0247 varies with an amplitude of up to $0.15 \mathrm{mag}$ but there is no longterm trend, although there is a possible modulation in the amplitude. The ASAS photometry, whether taken as a whole or subdivided into shorter intervals, or supplemented by the SAAO and P7 data, consistently indicates periods of 22.5 to 22.8 days, but with low significance. This period does not give a satisfactory light curve for any of these datasets, even for the data obtained in a single season: there is only a weak tendency toward a variation with phase in the derived period. Detailed examination of the time series for individual seasons shows great variability in

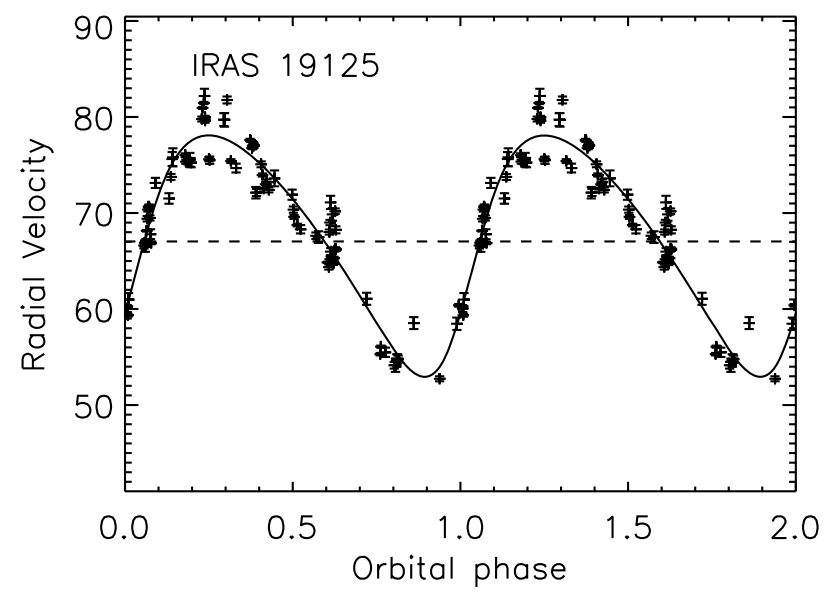

Fig. 13. The radial velocity data are folded at the orbital period of IRAS $19125+0343,519.6$ days. The full line is the orbital model.

both amplitude and length of successive cycles. The data are undersampled for this star, making delineation of the variability difficult. Kiss et al. (2007) found an irregular variation, with a possible periodicity of 25 days.

We have 111 radial velocity measurements over a time range of 3397 days. Both PDM and a Fourier analysis indicate that the 120 day period is significant. This period in radial velocity is not present in the photometry and we interpret this as the orbital period. The non-pulsational origin of the 120 day period is corroborated by two arguments. First, 120 days is a very long pulsational period for an F-type post-AGB star, and in any case it is not found in the photometric analysis. Secondly, the peakto-peak variations in $V$ for IRAS $19157-0247$ average 0.13 mag at most, so it is very doubtful that the radial velocity variations with a peak-to-peak amplitude of $24 \mathrm{~km} \mathrm{~s}^{-1}$ are only due to pulsations. Interpreting the velocity curve as being produced by pulsations of the photosphere, the integrated velocity would yield a radius increase of about half the initial radius. Such a pulsation would clearly not go unnoticed in the photometry. We therefore interpret the velocity variations as being caused by orbital motion. This is a very short orbital period with a high eccentricity placing strong constraints on the evolutionary history of the star!

Figure 14 shows the radial velocities and the residuals folded at this period. We found an eccentricity of 0.31 . The other orbital elements are shown in Table 7. The non-zero eccentricity was tested with the classical Lucy and Sweeney test (Lucy \& Sweeney 1971). The value of $\sum(\mathrm{O}-\mathrm{C})^{2}$ is $14 \%$ higher for a circular fit. This, together with the many cycles sampled, shows that the eccentricity is significant. The periodograms for the residual velocities show several peaks between 9 and 20 days, probably related to the timescale of the pulsations. None of them, however, lead to a significant variance reduction.

\section{Discussion}

\subsection{Orbits}

The most important conclusion of this extensive monitoring effort is that we have proven that all the stars in the programme are binaries. Unsurprisingly, given the high luminosities of the primaries, they are single-lined spectroscopic binaries because we have found no evidence in the spectra of flux contributions from a companion.

The orbital elements cover a wide range of periods, from 120 to 1500 days, for a range in mass functions of 0.004 to $0.57 M_{\odot}$. 


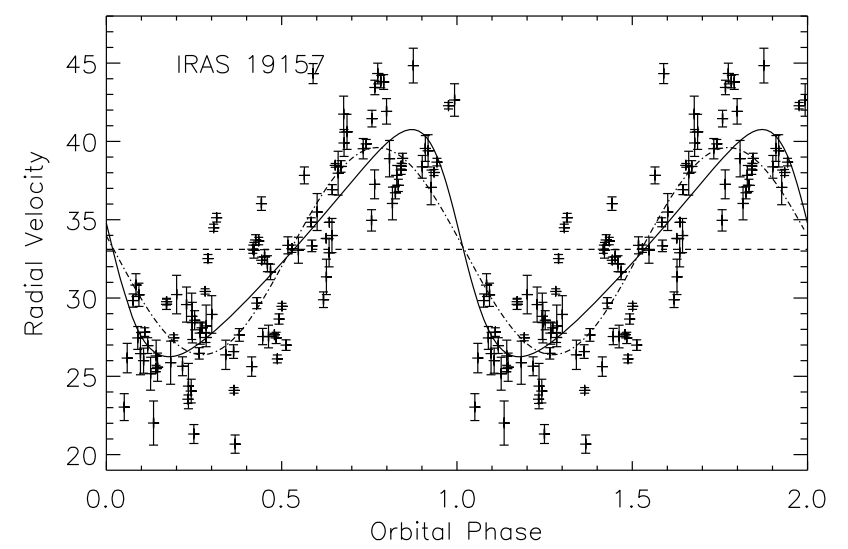

Fig. 14. The radial velocities of IRAS 19157-0247 folded at the orbital period of 119.5 days. The full line is the best-fit orbital model, the dashed-dotted line is the circular model. The horizontal line indicates the system velocity.

Table 8. The evaluation of the mass of the invisible companion of every programme star.

\begin{tabular}{lll}
\hline \hline Star & \multicolumn{2}{l}{ Mass of the companion in solar mass. } \\
& $i=90^{\circ}$ & $i=65^{\circ}$ \\
\hline IRAS 08544-4431 & 0.29 & 0.33 \\
IRAS 12222-4652 & 0.13 & 0.15 \\
EN TrA & 1.25 & 1.50 \\
IRAS 15469-5311 & 0.41 & 0.47 \\
IRAS 19125+0343 & 0.49 & 0.56 \\
IRAS 19157-0247 & 0.13 & 0.15 \\
\hline
\end{tabular}

The semi-major axes of the orbits of the primaries around the centre of mass cover the range $a \sin i=0.08$ to $2.1 \mathrm{AU}$. These are rather small orbits compared to the dimensions of a single AGB star. With their present estimated luminosity, these stars were too large while on the AGB to fit within their orbits and they must have been subject to severe binary interaction.

The physical interpretation of the orbital elements is hampered by the unknown inclination. Provided the evolved component has a remaining canonical mass of $0.6 M_{\odot}$, the minimal masses of the companions are given in Table 8 as well as the masses obtained assuming an inclination of $65^{\circ}$.

It is, however, very unlikely that we observe the objects nearly edge-on. To quantify the impact of the inclination on the observed SED and observed line-of-sight reddening, we used the detailed radiative transfer model of the disc around IRAS 08544 4431 (Deroo et al. 2007) and assumed that a similar model applies to all programme stars. The inner hole is dust free since we assume that dust grains cannot exist above the sublimation temperature. The presence of this inner gap allows the existence of a vertical boundary directly irradiated by the central star, creating a puffed-up geometry (Deroo et al. 2007). The scale height of the disc must be significant in all objects because of the very significant near infrared luminosity in the entire sample (de Ruyter et al. 2006). The temperature, density, and scale height of the disc are calculated based on the condition of hydrostatic equilibrium, and iterated to obtain the convergent structure. The constraints on the model parameters of the disc around IRAS 08544 4431 were taken from spectrally resolved interferometric data in both the near-IR and the $N$-band (Deroo et al. 2007).

In Fig. 15, we show the impact of different inclinations on the SED. The figure illustrates that around an inclination of $70^{\circ}$, the line-of-sight towards the central object will graze the

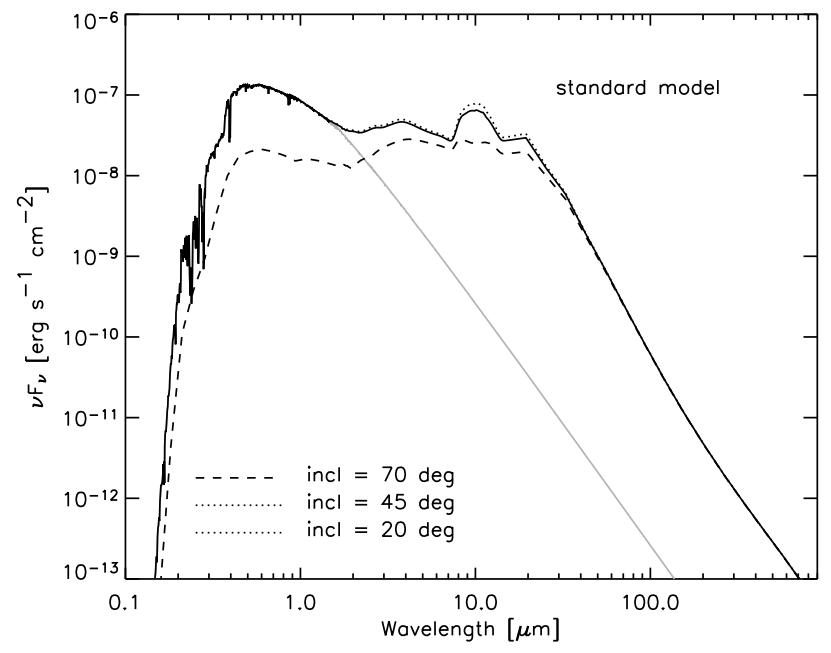

Fig. 15. The impact of the inclination on the SED computed by radiative transfer using typical parameters for a passively irradiated disc. The lower inclinations coincide on this scale so the same dotted line is used.

disc. This will strongly affect the circumstellar reddening. The line-of-sight circumstellar reddening in the $70^{\circ}$ model is about $E(B-V)=0.8$. Edge-on, the discs are strongly opaque and no direct visible light of the central star will reach us. The objects with the largest reddening are IRAS 08544-4431, IRAS 154695311, and IRAS $19125+0343$ with $E(B-V)=1.5 \pm 0.3,1.5 \pm$ 0.3 , and $1.2 \pm 0.3$, respectively (de Ruyter et al. 2006). The low Galactic latitudes and the strong interstellar DIBs in their spectra (Fig. 3 of Maas et al. 2005) indicate, however, that the greater part is of interstellar origin. We conclude that in all six objects, the inclination of the orbit is very likely to be less than or equal to $65^{\circ}$ and the corresponding lower limits to the mass estimates are also given in Table 8. EN TrA is the only object for which we have direct evidence that the companion is too massive to be a white dwarf. We also note that for the other objects, there is no evidence of a hot white dwarf, in neither the energetics nor the lack of spectroscopic evidence of symbiotic activity. The companion stars are likely to be unevolved main-sequence stars, which do not contribute significantly to the energy budget of the systems.

\subsection{Chemical composition}

A surprising effect of the presence of a disc is that the photospheric chemical content can be strongly affected by depletion (Van Winckel et al. 1992; Waters et al. 1992). The observed chemical pattern in the photospheres is the result of gas-dust separation followed by re-accretion of only the gas, which is poor in refractory elements. The photospheres are then deficient in refractories (such as Fe and $\mathrm{Ca}$ and the s-process elements), while the non-refractories are not affected. Photospheric depletion is surprisingly common in evolved objects (Giridhar et al. 2005; Maas et al. 2005, 2007, and references therein). We also found that depletion is present in the LMC (Reyniers \& van Winckel 2007), again around objects where the presence of a gravitationally bound disc is suspected.

Here we strengthen the link between the efficiency of the depletion process and the binary nature of the object. IRAS 085444431, IRAS 15469-5311, IRAS 19125+0343, and, to a much lesser extent, IRAS 19157-0247 are affected by photospheric depletion (Maas et al. 2005). EN TrA is iron deficient but the signature of depletion is less clear, while IRAS 12222-4652 
has mainly solar abundances (Van Winckel 1997). The depletion process will mask third dredge-up chemical enrichment since the s-process elements are strongly refractory (Lodders 2003). In none of these objects is there any evidence of AGB nucleosynthetic enrichment.

Infrared spectra of EN TrA, IRAS 19125+0343, and IRAS 19157-0247 were presented by Gielen et al. (2008), and for IRAS 08544-4431 by Deroo et al. (2007). In all objects, the dust features are dominated by silicates and characterised by a very strong degree of processing both in grainsize and crystallinity. The dust disc creation occurred when the envelope was O-rich. This is a general characteristic of the entire sample (Van Winckel 2003) and the chemical evidence of both the photospheres and the circumstellar material show that the AGB evolution of those binaries was cut short by the phase of strong binary interaction.

\subsection{Binary evolution}

Calculations from Schuerman (1972) show that, when one takes into account the radiation pressure of the AGB-star in a close binary system, the critical Roche potential degenerates into a surface containing the inner Lagrangian point $L_{1}$ and the outer Lagrangian point $L_{2}$ for a critical ratio of the radiation pressure force to the gravitational attraction. For this critical ratio, material lost by the AGB star flows through the $L_{1}$ point and can settle itself through the $L_{2}$ point into a circumbinary disc (see also Frankowski \& Jorissen 2007).

Population synthesis experiments (Bonačić Marinović et al. 2008) account for tidally enhanced mass-loss (Tout \& Eggleton 1988) during AGB evolution and balance the eccentricity pumping mechanisms induced by a prescription of the mass-loss that is orbital phase related, so that at periastron passage, mass loss is enhanced (Soker 2000). The conclusion of Bonačić Marinović et al. (2008) was that the eccentricity enhancement process is in balance with the circularisation for objects that do not enter the common envelope phase and are expected to leave the AGB in orbits of around 1000 days or longer. The eccentric orbits with shorter periods (those of IRAS 08544-4431, IRAS 154695311, IRAS 19125+0343, and especially IRAS 19157-0247) are still not accounted for. In these systems, additional eccentricity pumping mechanisms should be at work which may be linked to the presence of the circumbinary disc (Waelkens et al. 1996; Artymowicz et al. 1991). Similar but less pronounced excess eccentricities with respect to theory are found in related objects (e.g., Jorissen et al. 2009). Despite the phase of strong binary interaction, there is no evidence that the mass transfer towards the undetected companion has been very efficient, the mass functions implying rather low minimal masses for the secondary (see Table 8).

The global picture that emerges from our radial velocity monitoring is that the post-AGB stars evolved in a system that is too small to accommodate a peak AGB star. During a poorly understood phase of strong interaction, which for small orbits was probably a common envelope phase, a circumbinary dusty disc was formed, but the binary system did not experience a dramatic decrease in orbital radius. What we observe now is an F-G supergiant in a binary system, which is surrounded by a circumbinary dusty disc in a bound orbit. Although not yet proven, the fact that we found a $100 \%$ binarity rate for our subsample of objects with low pulsational amplitude strongly favours the binary nature of all post-AGB stars with a hot dust component.

The formation, structure, and evolution of the disc are far from being understood, but do appear to be key ingredients in our understanding of the late evolution of a very significant binary population.

\subsection{Variability}

These six stars exhibit a wide range of properties, in particular in terms of amplitude and, especially, the regularity of variation. The first three stars, IRAS 08544-4431, IRAS 12222-4652, and EN TrA, have periodograms with peaks which stand out strongly from the noise, while IRAS 15469-5311 has a much weaker peak and IRAS 19125+0343 and IRAS 19157-0247 exhibit only a confused scatter, with minor peaks on a noisy background. IRAS $08544-4431$ is the only one of the six stars to exhibit clearly defined, coherent variations for as long as one or two years. IRAS 12222-4652 and EN TrA have better defined peaks in their periodogram than IRAS 08544-4431, with a single period in the case of EN TrA, but their amplitudes and, in the case of IRAS 12222-4652, the lengths of individual cycles change continuously. The variations of IRAS 15469-5311 and IRAS $19125+0343$ exhibit cycles of very variable amplitude and length, with evidence of changes in the direction of pulsation within a given cycle for IRAS 19125+0343, while the data for IRAS 19157-0247 are inadequate to elucidate the nature of the variations, which are probably even more irregular and of relatively short period.

These stars are of earlier spectral type and so are hotter than classical RV Tauri stars (Lloyd Evans 1999) and appear therefore to lie toward the blue side of the Cepheid instability strip. This raises a question about the nature of their pulsation. The ratio of the two principal periods is 0.96 for IRAS $08544-4431$ and 0.91 for IRAS 12222-4652, whereas the ratio of the first overtone to the fundamental, $\mathrm{P} 1 / \mathrm{P} 0$, is always close to 0.705 in classical Cepheids (Stobie 1977). Two high overtones might have the period ratios that we observe, but it seems implausible that these would be dominant, and the periods of these two stars are quite long, implying very long fundamental periods and a correspondingly high luminosity. This suggests that the pulsations of these stars are non-radial. EN TrA, which has a single, shorter, period and a larger amplitude, is, however, probably a radial pulsator. The apparently abrupt turnaround in the direction of the change in $V$ magnitude, leading to a second maximum and a consequent lengthening of individual cycles in IRAS 19125+0343, recalls the suggestion that shockwaves may be involved in the complex motions of the outer atmosphere of the post-AGB star HD 56126 (Barthès et al. 2000).

The four stars of largest amplitude showed indications of the classical RV Tauri feature of alternating deep and shallow minima, but an equally large alternation of the magnitude at successive maxima.

Multicolour observations may be used to compare light and colour curves, which follow a similar phase relationship in classical Cepheids but which differ in type II Cepheids, for which an atmospheric shock leads to a bluer colour on the rise to maximum light, with wide loops in two-colour diagrams, whereas classical Cepheids have very narrow loops. The amount by which the $\mathrm{B}$ curve leads the $\mathrm{V}$ curve, in terms of relative phase in the single period, is: IRAS 08544-4431, 0.00:, IRAS 122224652, 0.04, EN TrA, 0.17. The scatter in the data and small amplitudes preclude any finding for the remaining stars. It is unfortunate that the SAAO observations of IRAS 08544-4431 were made when the amplitude was relatively small. It is only for EN TrA, the star of largest amplitude, that the phase shift typical of type II Cepheids is clearly seen. 


\section{Conclusions}

The summary of the main conclusions of this contribution is that:

- Our radial velocity monitoring show that post-AGB stars that have both a hot dust component (and hence a disc) and a low photospheric pulsational amplitude (de Ruyter et al. 2006), are binaries. Even in these objects, the amplitude of the radial velocity variations induced by the pulsation is a significant fraction of the orbital velocity. It is an observational challenge to prove the binary nature of all post-AGB objects with a hot dust component, because in many systems large amplitude pulsations are present. Nevertheless, that $100 \%$ of our low amplitude pulsator programme stars are binaries is a strong indication that binarity is a prerequisite for disc formation.

- We confirm and strengthen the conclusion of Kiss et al. (2007) that complex multi-periodic but low-amplitude pulsational behaviour is present at the blue side of the type II Cepheid instability strip.

- The evolution of the binaries in the resulting periodeccentricity parameter space is not understood by using standard binary evolution channels. Theoretical efforts have included enhanced mass-loss and an efficient eccentricity pumping mechanism induced by modulation of the massloss in the orbital period. Nevertheless, the high eccentricity of IRAS 08544-4431, IRAS $19125+0343$ and especially IRAS 19157-0247 (with a period of 120 days and a significant non-zero eccentricity) are detected in systems that are very likely to be post-common envelope systems and in these systems, the high eccentricity remains very poorly understood. The (on average) small mass functions imply that mass transfer to the companion has been limited.

- In all systems found so far, the dust in the disc is O-rich. The AGB evolution was likely cut short by the binary interaction event and with the orbital elements found in this work, the systems should be seen as post-interacting binaries rather than canonical post-AGB stars.

- The wide range of orbits and mass function detected here show that the creation of a circumbinary gravitationally bound disc is the final evolutionary product of a wide range of binaries.

Acknowledgements. The authors want to acknowledge the Geneva Observatory and its staff for the generous time allocation on the Swiss Euler telescope. The IvS acknowledges support from the Fund for Scientific Research of Flanders (FWO) under the grants G.0178.02., G.0703.08, G.0332.06 and G.0470.07. This research was made possible thanks to support from the Research Council of K.U.Leuven under grant GOA/2008/04. We would like to express our appreciation to Grzegorz Pojmanski for his efforts to provide and maintain the ASAS data in a form accessible to the astronomical community.

\section{References}

Artymowicz, P., Clarke, C. J., Lubow, S. H., \& Pringle, J. E. 1991, ApJ, 370, L35

Balick, B., \& Frank, A. 2002, ARA\&A, 40, 439

Baranne, A., Mayor, M., \& Poncet, J. L. 1979, Vistas Astron., 23, 279

Barthès, D., Lèbre, A., Gillet, D., \& Mauron, N. 2000, A\&A, 359, 168

Berdnikov, L. N., \& Turner, D. G. 1995, Pis'ma Astronomich. Zh., 21, 603

Bertiau, F. C., \& Grobben, J. 1969, Ricerche Astron., 8, 1

Bonačić Marinović, A. A., Glebbeek, E., \& Pols, O. R. 2008, A\&A, 480, 797

Bujarrabal, V., Castro-Carrizo, A., Alcolea, J., \& Neri, R. 2005, A\&A, 441, 1031

Caldwell, J. A. R., Coulson, I. M., Dean, J. F., \& Berdnikov, L. N. 2001, J. Astron. Data, 7, 4

Cohen, M., Van Winckel, H., Bond, H. E., \& Gull, T. R. 2004, AJ, 127, 2362
De Ruyter, S., Van Winckel, H., Dominik, C., Waters, L. B. F. M., \& Dejonghe, H. 2005, A\&A, 435, 161

de Ruyter, S., van Winckel, H., Maas, T., et al. 2006, A\&A, 448, 641

Deroo, P., van Winckel, H., Min, M., et al. 2006, A\&A, 450, 181

Deroo, P., Acke, B., Verhoelst, T., et al. 2007, A\&A, 474, L45

Eggen, O. J. 1986, AJ, 91, 890

Fokin, A. B., Lèbre, A., Le Coroller, H., \& Gillet, D. 2001, A\&A, 378, 546

Frankowski, A., \& Jorissen, A. 2007, Baltic Astron., 16, 104

Gauba, G., Parthasarathy, M., Kumar, B., Yadav, R. K. S., \& Sagar, R. 2003, A\&A, 404, 305

Gielen, C., van Winckel, H., Waters, L. B. F. M., Min, M., \& Dominik, C. 2007, A\&A, 475, 629

Gielen, C., van Winckel, H., Min, M., Waters, L. B. F. M., \& Lloyd Evans, T. 2008, A\&A, 490, 725

Giridhar, S., Lambert, D. L., Reddy, B. E., Gonzalez, G., \& Yong, D. 2005, ApJ, 627,432

Golay, M. 1980, Vistas Astron., 24, 141

Grayzeck, E. J. 1978, AJ, 83, 1397

Hadrava, P. 2004, Publ. Astron. Inst. Czechoslovak Acad. Sci., 92, 1

Houk, N., Hartoog, M. R., \& Cowley, A. P. 1976, AJ, 81, 116

Jorissen, A., Frankowski, A., Famaey, B., \& Van Eck, S. 2009, arXiv e-prints

Kiss, L. L., Derekas, A., Szabó, G. M., Bedding, T. R., \& Szabados, L. 2007, MNRAS, 375, 1338

Kwok, S., Volk, K., \& Bidelman, W. P. 1997, ApJS, 112, 557

Le Squeren, A. M., Sivagnanam, P., Dennefeld, M., \& David, P. 1992, A\&A, 254, 133

Lloyd Evans, T. 1985, MNRAS, 217, 493

Lloyd Evans, T. 1999, in Asymptotic Giant Branch Stars, IAU Symp., 191, 453

Lodders, K. 2003, ApJ, 591, 1220

Lucy, L. B., \& Sweeney, M. A. 1971, AJ, 76, 544

Maas, T., Van Winckel, H., \& Waelkens, C. 2002, A\&A, 386, 504

Maas, T., Van Winckel, H., Lloyd Evans, T., et al. 2003, A\&A, 405, 271

Maas, T., Van Winckel, H., \& Lloyd Evans, T. 2005, A\&A, 429, 297

Maas, T., Giridhar, S., \& Lambert, D. L. 2007, ApJ, 666, 378

MacConnell, D. J., \& Bidelman, W. P. 1976, AJ, 81, 225

Martinez, P., Kilkenny, D. M., Cox, G., et al. 2002, Monthly Notes of the Astronomical Society of South Africa, 61, 102

Men'shchikov, A. B., Schertl, D., Tuthill, P. G., Weigelt, G., \& Yungelson, L. R. 2002, A\&A, 393, 867

Menzies, J. W., Cousins, A. W. J., Banfield, R. M., \& Laing, J. D. 1989, South African Astronomical Observatory Circ., 13, 1

Miszalski, B., Acker, A., Moffat, A. F. J., Parker, Q. A., \& Udalski, A. 2009, A\&A, 496, 813

Moe, M., \& De Marco, O. 2006, ApJ, 650, 916

Parthasarathy, M., Vijapurkar, J., \& Drilling, J. S. 2000, A\&AS, 145, 269

Pel, J. W. 1976, A\&AS, 24, 413

Perryman, M. A. C., \& ESA 1997, The HIPPARCOS and TYCHO catalogues. Astrometric and photometric star catalogues derived from the ESA HIPPARCOS Space Astrometry Mission, ESA SP, 1200

Pickering, E. C. 1907, Astron. Nachr., 175, 333

Pojmanski, G. 2002, Acta Astron., 52, 397

Queloz, D., Casse, M., \& Mayor, M. 1999, in Precise Stellar Radial Velocities, ed. J. B. Hearnshaw, \& C. D. Scarfe, IAU Colloq., 170, ASP Conf. Ser., 185, 13

Raskin, G., Burki, G., Burnet, M., et al. 2004, in SPIE Conf. Ser., 5492, 830

Reyniers, M., \& Cuypers, J. 2005, A\&A, 432, 595

Reyniers, M., \& van Winckel, H. 2007, A\&A, 463, L1

Sahai, R., Morris, M., Sánchez Contreras, C., \& Claussen, M. 2007, AJ, 134, 2200

Schuerman, D. W. 1972, Ap\&SS, 19, 351

Soker, N. 2000, A\&A, 357, 557

Stellingwerf, R. F. 1978, ApJ, 224, 953

Stobie, R. S. 1977, MNRAS, 180, 631

Szczerba, R., Siódmiak, N., Stasińska, G., \& Borkowski, J. 2007, A\&A, 469, 799

Tout, C. A., \& Eggleton, P. P. 1988, MNRAS, 231, 823

Van Winckel, H. 1997, A\&A, 319, 561

Van Winckel, H. 2003, ARA\&A, 41, 391

Van Winckel, H. 2007, Baltic Astron., 16, 112

Van Winckel, H., Mathis, J. S., \& Waelkens, C. 1992, Nature, 356, 500

Van Winckel, H., Waelkens, C., Waters, L. B. F. M., et al. 1998, A\&A, 336, L17

Van Winckel, H., Waelkens, C., Fernie, J. D., \& Waters, L. B. F. M. 1999, A\&A, 343, 202

Waelkens, C., Van Winckel, H., Waters, L. B. F. M., \& Bakker, E. J. 1996, A\&A, 314, L17

Waters, L. B. F. M., Trams, N. R., \& Waelkens, C. 1992, A\&A, 262, L37

Zijlstra, A. A. 2007, Baltic Astron., 16, 79 
H. Van Winckel et al.: Binarity of post-AGB stars, Online Material p 1

Table 4. Individual heliocentric radial velocity datapoints of the programme stars.

\begin{tabular}{|c|c|c|c|c|c|c|c|c|}
\hline$\overline{\mathrm{JD}(24+)}$ & $\overline{\bar{V}(\mathrm{rad})}$ & $\overline{\sigma \sigma}$ & $\overline{\mathrm{JD}(24+)}$ & $\overline{\bar{V}(\mathrm{rad})}$ & $\overline{\sigma \sigma}$ & $\overline{\mathrm{JD}(24+)}$ & $\overline{\bar{V}(\mathrm{rad})}$ & $\sigma$ \\
\hline \multicolumn{9}{|c|}{ IRAS 08544-4431 } \\
\hline 51146.7632 & 53.93 & 0.46 & 52019.5475 & 65.09 & 0.82 & 52736.5670 & 55.73 & 0.45 \\
\hline 51275.5548 & 58.82 & 0.67 & 52020.5660 & 64.57 & 0.89 & 52737.5675 & 55.23 & 0.66 \\
\hline 51276.5400 & 58.41 & 0.83 & 52025.5503 & 62.85 & 0.89 & 52738.5715 & 55.44 & 0.35 \\
\hline 51278.5411 & 58.55 & 0.69 & 52026.5400 & 62.50 & 0.88 & 52740.6439 & 54.65 & 0.34 \\
\hline 51282.5516 & 59.17 & 0.71 & 52227.8345 & 54.60 & 0.36 & 52983.7815 & 73.75 & 0.18 \\
\hline 51284.5721 & 59.32 & 0.65 & 52229.8411 & 55.27 & 0.36 & 52985.8487 & 75.66 & 0.91 \\
\hline 51467.8209 & 69.46 & 0.72 & 52230.7878 & 55.48 & 0.47 & 52986.7656 & 73.35 & 0.28 \\
\hline 5146 & 68.67 & 0.63 & 52231.8190 & 55.92 & 0.45 & 52987 & 72.92 & 0.20 \\
\hline 51510.8488 & 66.78 & 0.88 & 52232.8366 & 56.32 & 0.47 & 52988.7097 & 72.75 & 0.32 \\
\hline 51593.7902 & 60.27 & 0.51 & 52233.8223 & 56.59 & 0.43 & 52989.7627 & 72.25 & 0.35 \\
\hline 51596.5369 & 60.64 & 0.91 & 52234.8312 & 56.93 & 0.43 & 52990.7121 & 71.86 & 0.38 \\
\hline 51600.5514 & 58.22 & 0.43 & 52235.8421 & 57.33 & 0.39 & 52991.7597 & 71.52 & 0.47 \\
\hline 51603.5344 & 57.93 & 0.32 & 52236.8460 & 57.39 & 0.44 & 52992.7407 & 71.04 & 0.47 \\
\hline 51652.6373 & 53.56 & 0.25 & 52237.8173 & 57.41 & 0.50 & 52993.7198 & 70.53 & 0.50 \\
\hline 51655.6467 & 54.94 & 0.51 & 52238.8600 & 57.51 & 0.47 & 52994.8236 & 69.88 & 0.61 \\
\hline 51659.5312 & 56.51 & 0.26 & 52239.8453 & 57.39 & 0.52 & 53072.6987 & 56.49 & 0.67 \\
\hline 51716.4844 & 54.94 & 0.45 & 52240.8067 & 57.35 & 0.56 & 53073.6075 & 56.03 & 0.70 \\
\hline 51722.5308 & 56.82 & 0.45 & 52272.7653 & 62.12 & 0.57 & 53077.6309 & 55.84 & 0.99 \\
\hline 51726.4784 & 57.69 & 0.42 & 52273.6877 & 62.32 & 0.42 & 53078.6744 & 55.59 & 0.59 \\
\hline 51728.5054 & 58.24 & 0.41 & 52274.7185 & 61.92 & 0.47 & 53079.6282 & 56.25 & 0.85 \\
\hline 51730.4723 & 58.43 & 0.42 & 52278.6249 & 61.41 & 0.37 & 53083.6303 & 27 & 0.72 \\
\hline 51732 & 58.61 & 0.48 & 52279.7142 & & 0.48 & & & 0.44 \\
\hline 51816.8902 & 64.86 & 0.37 & 52280.5488 & 61 & 5 & 53 & & 0.19 \\
\hline 518 & 64 & & 52281.7 & 62.11 & 0 & 53 & & 0.80 \\
\hline 51818.9059 & 63 & 0. & 52282.6908 & 62.63 & 0. & 531 & & 0.64 \\
\hline 51819.8806 & 64 & 0.40 & 52282.6914 & 62.48 & 0 & 531 & 7 & 0.37 \\
\hline 51820.8975 & 63.12 & 0.8 & 52283.5876 & 62.60 & 0.33 & 531 & 4 & 0.49 \\
\hline 51821.8891 & 62.68 & 0.55 & 52284.6146 & 62.81 & 0.22 & 53185.4616 & 57.85 & 0.78 \\
\hline 51822.8886 & 62.80 & 0.41 & 52285.7966 & 63.59 & 0.33 & 53187.4640 & 56.03 & 0.61 \\
\hline 51823.8627 & 62.33 & 0.43 & 52285.8736 & 62.98 & 0.40 & 53354.7158 & 65.77 & 0.66 \\
\hline 51824.8883 & 62.10 & 0.53 & 52329.6329 & 62.15 & 0.71 & 53356.6942 & 64.76 & 0.70 \\
\hline 51825.8752 & 61.63 & 0.51 & 52330.6253 & 62.01 & 0.95 & 53358 & 63.70 & 0.78 \\
\hline 51826.8900 & 61.49 & 0.55 & 52331.6184 & 61.97 & 0.83 & 53360 & 62.70 & 0.71 \\
\hline 51827.8881 & 61. & 9 & 52332.6912 & 62.09 & & 533 & 6 & 0.81 \\
\hline 518 & & & 52333.5833 & 61 & 0.94 & & & 0.39 \\
\hline 5182 & 61 & & 52334.6 & & 0.73 & & & 0.35 \\
\hline 5188 & & & 91 & & 37 & & & 0.14 \\
\hline 518 & & & & & 1 & & & 0.50 \\
\hline 518 & & 4 & 52337. & & 0 & & & 0.86 \\
\hline 518 & 71 & 9 & 52338.5 & 63.03 & 0.85 & 537 & 77 & 0.28 \\
\hline 518 & 72. & 3 & 52339.5910 & 63.10 & 0.71 & 537 & 1 & 0.49 \\
\hline 51891.8222 & 72.72 & 0.51 & 52340.6162 & 62.78 & 0.69 & 53713.7763 & 53.82 & 0.67 \\
\hline 51892.8058 & 73.43 & 0.48 & 52341.5897 & 63.06 & 0.79 & 53833.6118 & 61.90 & 0.36 \\
\hline 51894.8195 & 73.29 & 0.67 & 52571.8377 & 59.18 & 0.93 & 53837.4779 & 62.28 & 0.58 \\
\hline 51940.7879 & 68.49 & 0.81 & 52581.8738 & 58.91 & 0.46 & 54077.8299 & 60.43 & 0.36 \\
\hline 51943.7737 & 69.84 & 0.6 & 52727.5427 & 54.28 & 0.52 & 54080.8300 & 60.65 & 0.18 \\
\hline 51944.5971 & 69.83 & 0.70 & 52728.5978 & 54.28 & 0.39 & 54180 & 55.01 & 0.39 \\
\hline 51948.6634 & 71.90 & .66 & 52729.5123 & 54.86 & 0.43 & 54183.5442 & & 0.38 \\
\hline 51 & 71.96 & 9 & 52730.5892 & 54.87 & 0.43 & 54 & 55.69 & 0.64 \\
\hline 51953.6671 & 71.86 & 0.63 & 52731.5081 & 55.54 & 0.34 & 54478.6732 & 75 & 0.33 \\
\hline 52015.5681 & 65.37 & 0.59 & 52732.5623 & & 0.43 & 54479.6564 & 73 & 0.40 \\
\hline 5201 & & 0.63 & & & 62 & & 69.43 & 0.40 \\
\hline & & 081 & 52734.5704 & & 0.50 & 54486.7220 & 69.69 & 0.45 \\
\hline 52018.5751 & 65.03 & 0.84 & 52735.5964 & 55.80 & 0.52 & & & \\
\hline \multicolumn{9}{|c|}{ IRAS 12222-4652 } \\
\hline & 7.4 & .5 & 49406.840 & 0.09 & .5 & & & 0.61 \\
\hline 7.857 & 2.35 & .5 & 49407.826 & -0.78 & .5 & 52469.6082 & & 0.36 \\
\hline 45881.510 & -1.29 & .5 & 49409.851 & -1.43 & .5 & 52729.7034 & 11.28 & 0.24 \\
\hline 47913.900 & 5.4 & 1.0 & 49522.582 & 0.90 & .5 & 52733.7608 & 10.55 & 0.35 \\
\hline 47914.900 & 2.8 & 1.0 & 49526.659 & 0.81 & .5 & 52736.7651 & 8.16 & 0.47 \\
\hline 47915.800 & 4.4 & 1.0 & 50527.749 & 3.7 & 1.0 & 52739.6906 & 4.48 & 0.20 \\
\hline 47915.800 & 5.8 & 1.0 & 50530.799 & 3.5 & 1.0 & 52804.5557 & 2.05 & 0.11 \\
\hline 47917.800 & 6.6 & 1.0 & 51594.8448 & 5.64 & 0.50 & 53073.7687 & 1.21 & 0.37 \\
\hline
\end{tabular}


H. Van Winckel et al.: Binarity of post-AGB stars, Online Material p 2

Table 4. continued.

\begin{tabular}{|c|c|c|c|c|c|c|c|c|}
\hline$\overline{\mathrm{JD}(24+)}$ & $\bar{V}(\mathrm{rad})$ & $\overline{\sigma \sigma}$ & $\overline{\mathrm{JDD}(24+)}$ & $\bar{V}(\mathrm{rad})$ & $\overline{\bar{\sigma}}$ & $\overline{\mathrm{JD}(24+)}$ & $\bar{V}(\mathrm{rad})$ & $\sigma$ \\
\hline 48289.868 & 5.67 & .5 & 51766.5384 & 7.91 & 0.49 & 53075.8142 & 1.01 & 0.37 \\
\hline 48312.794 & 0.07 & .5 & 51768.5061 & 6.09 & 0.44 & 53076.8279 & 0.85 & 0.28 \\
\hline 48317.694 & -0.12 & .5 & 51769.5234 & 5.43 & 0.38 & 53078.8192 & 0.23 & 0.21 \\
\hline 48400.651 & 3.41 & .5 & 51771.4884 & 5.27 & 0.53 & 53080.7999 & 0.33 & 0.26 \\
\hline 48453.476 & 1.74 & .5 & 51772.4816 & 5.40 & 0.60 & 53085.7984 & 0.89 & 0.27 \\
\hline 48460.500 & 1.79 & .5 & 51945.7182 & 5.28 & 0.56 & 53178.5860 & -1.44 & 0.27 \\
\hline 48648.885 & 2.33 & .5 & 52016.7528 & 1.86 & 0.13 & 53182.6712 & -2.26 & 0.38 \\
\hline 49006.800 & 8.9 & 1.0 & 52018.7552 & 2.25 & 0.18 & 53185.6080 & -2.15 & 0.23 \\
\hline 49031.800 & 5.8 & 1.0 & 52021.7249 & 1.88 & 0.20 & 53188.4618 & -0.87 & 0.26 \\
\hline 49054.811 & 7.97 & .5 & 52024.6860 & 1.05 & 0.15 & 53189.4601 & -0.64 & 0.29 \\
\hline 49099.700 & 5.4 & 1.0 & 52027.7294 & 1.69 & 0.15 & 53399.8023 & 6.85 & 0.59 \\
\hline 49121.600 & 3.4 & 1.0 & 52111.5528 & 2.93 & 0.40 & 53402.8788 & 7.68 & 0.21 \\
\hline 49122.600 & 6.4 & 1.0 & 52114.5745 & 1.98 & 0.40 & 53475.7560 & 4.39 & 0.59 \\
\hline 49122.700 & 4.8 & 1.0 & 52115.5823 & 1.58 & 0.20 & 53478.7847 & 5.10 & 0.32 \\
\hline 49172.529 & 1.96 & .5 & 52118.5568 & 0.67 & 0.27 & 53481.7560 & 5.61 & 0.89 \\
\hline 49189.500 & 7. & 1.0 & 52122.5608 & -0.70 & 0.17 & 53483.7766 & 5.04 & 0.24 \\
\hline 49190.600 & 7.6 & 1.0 & 52329.8192 & 2.34 & 0.58 & 53808.7140 & 8.26 & 0.71 \\
\hline 49191.500 & 8.5 & 1.0 & 52332.8234 & 2.26 & 0.68 & 53830.7574 & 2.47 & 1.04 \\
\hline 49334.800 & -1.1 & 1.0 & 52334.7261 & 2.18 & 0.60 & 53833.7463 & 1.40 & 0.88 \\
\hline 49404.830 & -1.42 & .5 & 52338.7713 & 2.64 & 0.53 & & & \\
\hline \multicolumn{9}{|c|}{ EN TrA } \\
\hline 48312.891 & -3.29 & 0.5 & 49478.646 & -29.4 & 1.0 & 52339.9001 & -25.78 & 0.16 \\
\hline 48324.812 & -2.18 & 0.5 & 49478.699 & -29.8 & 1.0 & 52417.6713 & -22.85 & 0.47 \\
\hline 48327.796 & -3.7 & 1. & 49485.839 & -24 & 1.0 & 52425.6327 & -26.47 & 0.23 \\
\hline 48329.854 & -7.3 & 1. & 49774.878 & -7.3 & 1.0 & 52728.6876 & -8.99 & 0.35 \\
\hline 48329.769 & -6.5 & 1. & 49798.846 & -9.26 & 0.5 & 52731.7055 & -8.60 & 0.55 \\
\hline 48329.855 & -6.5 & 1. & 49831.822 & -4 & 1.0 & 52803.6081 & 0.20 & 0.44 \\
\hline 48456.520 & -0.07 & 0.5 & 49953.507 & -2.0 & 1.0 & 52817.5814 & -1.58 & 0.38 \\
\hline 48459.524 & 3.30 & 0.5 & 49967.470 & 7.4 & 1.0 & 53072.8237 & -4.37 & 0.24 \\
\hline 48460.518 & 5.12 & 0.5 & 50168.870 & -0.9 & 1.0 & 53073.7795 & -3.75 & 0.27 \\
\hline 48461.513 & 5.24 & 0.5 & 50189.870 & 10.2 & 1.0 & 53078.8486 & 0.23 & 0.22 \\
\hline 48462.506 & 6.69 & 0.5 & 50263.677 & 13.8 & 1.0 & 53084.8179 & 5.47 & 0.33 \\
\hline 48732.817 & 5.9 & 1.0 & 50276.5543 & 2.55 & 0.5 & 53179.6833 & 2.12 & 0.35 \\
\hline 48842.506 & 2.1 & 1.0 & 50496.865 & -0.5 & 1.0 & 53182.7142 & 2.23 & 0.04 \\
\hline 48844.516 & -0.3 & 1.0 & 50512.858 & -1.5 & 1.0 & 53185.6862 & 2.09 & 0.16 \\
\hline 48845.545 & 0.6 & 1.0 & 50526.894 & 5.4 & 1.0 & 53476.7417 & -2.14 & 0.36 \\
\hline 48845.511 & 0.5 & 1.0 & 50530.878 & 5.1 & 1.00 & 53481.7673 & 0.23 & 0.38 \\
\hline 48846.515 & 0.6 & 1.0 & 50532.901 & 3.2 & 1.00 & 53830.7692 & -21.91 & 0.45 \\
\hline 49031.842 & -2.5 & 1.0 & 52329.8257 & -29.35 & 0.33 & 53833.7578 & -26.71 & 0.31 \\
\hline 49099.765 & -0.6 & 1.0 & 52332.8298 & -29.26 & 0.06 & 53837.6745 & -31.87 & 0.70 \\
\hline 49121.681 & -3.7 & 1.0 & 52335.7537 & -28.33 & 0.08 & 53894.5700 & -25.87 & 0.46 \\
\hline 49188.565 & -11.9 & 1.0 & 52337.8864 & -27.22 & 0.14 & 53896.7059 & -24.65 & 0.84 \\
\hline \multicolumn{9}{|c|}{ IRAS 15469-5311 } \\
\hline 51277.7428 & -23.28 & 0.50 & 52115.6775 & -9.76 & 0.70 & 53074.8473 & -17.24 & 0.27 \\
\hline 51278.6732 & -23.20 & 0.55 & 52116.7262 & -9.94 & 0.49 & 53075.8342 & -16.32 & 0.21 \\
\hline 51469.4940 & -5.99 & 0.23 & 52117.5683 & -9.07 & 0.47 & 53076.8545 & -16.92 & 0.24 \\
\hline 51471.5005 & -6.71 & 0.38 & 52118.6009 & -8.90 & 0.24 & 53077.8545 & -17.00 & 0.20 \\
\hline 51478.5067 & -10.32 & 0.58 & 52122.5860 & -10.05 & 0.19 & 53078.8712 & -16.61 & 0.65 \\
\hline 51593.8952 & -27.10 & 0.49 & 52123.6856 & -10.10 & 0.15 & 53079.8356 & -17.57 & 0.38 \\
\hline 51595.8875 & -26.71 & 0.19 & 52278.8688 & -12.72 & 0.48 & 53081.8432 & -17.80 & 0.20 \\
\hline 51597.8803 & -26.39 & 0.35 & 52279.8866 & -11.88 & 0.31 & 53083.8381 & -19.06 & 0.35 \\
\hline 51599.8606 & -25.19 & 0.42 & 52280.8549 & -13.11 & 0.52 & 53084.8360 & -16.68 & 0.36 \\
\hline 51601.8871 & -24.05 & 0.47 & 52281.8797 & -13.64 & 0.18 & 53085.8280 & -17.15 & 0.11 \\
\hline 51603.8850 & -23.69 & 0.83 & 52283.8831 & -15.29 & 0.25 & 53182.7423 & -26.95 & 0.45 \\
\hline 51652.7351 & -22.93 & 0.23 & 52284.8682 & -16.01 & 0.38 & 53183.6508 & -26.38 & 0.33 \\
\hline 51652.7553 & -22.46 & 0.09 & 52285.8629 & -15.71 & 0.38 & 53184.6563 & -27.14 & 0.78 \\
\hline 51654.7638 & -22.67 & 0.31 & 52329.8361 & -20.85 & 0.36 & 53185.6962 & -26.24 & 0.48 \\
\hline 51659.7827 & -21.98 & 0.42 & 52330.8421 & -21.35 & 0.14 & 53187.6351 & -23.49 & 0.79 \\
\hline 51716.5979 & -14.11 & 0.31 & 52331.8053 & -21.56 & 0.35 & 53228.5658 & -19.17 & 0.25 \\
\hline 51718.6738 & -14.00 & 0.27 & 52333.8440 & -21.91 & 0.12 & 53230.6595 & -18.30 & 0.20 \\
\hline 51729.7062 & -12.17 & 0.43 & 52334.7942 & -22.23 & 0.11 & 53231.5788 & -18.26 & 0.21 \\
\hline 51730.7659 & -11.77 & 0.34 & 52336.8282 & -21.36 & 0.52 & 53232.5444 & -17.87 & 0.05 \\
\hline 51732.7620 & -11.74 & 0.33 & 52337.7946 & -22.11 & 0.27 & 53391.8499 & -3.47 & 0.76 \\
\hline 51760.5349 & -4.76 & 0.45 & 52338.8093 & -24.81 & 0.56 & 53397.7728 & -4.73 & 0.68 \\
\hline
\end{tabular}


H. Van Winckel et al.: Binarity of post-AGB stars, Online Material p 3

Table 4. continued.

\begin{tabular}{|c|c|c|c|c|c|c|c|c|}
\hline$\overline{\mathrm{JD}(24+)}$ & $\bar{l} V(\mathrm{rad})$ & $\bar{\sigma}$ & $\overline{\mathrm{JD}(24+)}$ & $\overline{V(\mathrm{rad})}$ & $\bar{\sigma}$ & $\overline{\mathrm{JD}(24+)}$ & $\overline{V(\mathrm{rad})}$ & $\bar{\sigma}$ \\
\hline 51761.4994 & -4.53 & 0.34 & 52339.7781 & -22.87 & 0.52 & 53399.7905 & -3.13 & 0.32 \\
\hline 51762.5343 & -4.48 & 0.36 & 52340.8450 & -25.11 & 0.21 & 53477.7892 & -20.06 & 0.66 \\
\hline 51762.5467 & -3.97 & 0.29 & 52341.7736 & -24.51 & 0.28 & 53479.7884 & -20.09 & 0.65 \\
\hline 51763.5447 & -3.87 & 0.40 & 52416.7697 & -24.46 & 0.11 & 53480.7505 & -20.16 & 0.53 \\
\hline 51765.5127 & -4.54 & 0.58 & 52424.6481 & -21.68 & 0.16 & 53482.7946 & -20.39 & 0.78 \\
\hline 51766.5339 & -4.27 & 0.62 & 52424.7176 & -22.57 & 0.24 & 53837.6853 & -11.32 & 0.47 \\
\hline 51768.5679 & -4.78 & 0.69 & 52468.6674 & -16.01 & 0.22 & 53894.5796 & -23.52 & 0.39 \\
\hline 51769.5646 & -3.94 & 0.85 & 52469.6336 & -15.60 & 0.21 & 53898.6560 & -22.85 & 0.54 \\
\hline 51940.8625 & -19.12 & 0.07 & 52470.6850 & -15.01 & 0.19 & 53903.6450 & -25.77 & 0.66 \\
\hline 51942.8880 & -20.90 & 0.17 & 52472.6732 & -14.31 & 0.19 & 54211.8782 & -14.91 & 0.48 \\
\hline 51943.8385 & -21.79 & 0.19 & 52473.6416 & -14.03 & 0.39 & 54219.8503 & -11.92 & 0.53 \\
\hline 51944.8526 & -23.03 & 0.35 & 52474.6498 & -13.30 & 0.14 & 54302.6379 & -31.31 & 0.46 \\
\hline 51945.8252 & -23.71 & 0.51 & 52481.6175 & -13.52 & 0.09 & 54304.5784 & -31.23 & 0.38 \\
\hline 51946.8458 & -23.71 & 0.82 & 52571.4983 & -5.83 & 0.32 & 54305.5780 & -30.75 & 0.46 \\
\hline 51948.8802 & -25.00 & 0.19 & 52727.7383 & -29.48 & 0.53 & 54310.5047 & -30.20 & 0.28 \\
\hline 51949.8475 & -25.11 & 0.47 & 52728.6970 & -29.44 & 0.26 & 54331.5914 & -24.90 & 0.28 \\
\hline 51950.8386 & -25.46 & 0.40 & 52729.7127 & -29.88 & 0.50 & 36.5006 & -24.51 & 0.47 \\
\hline 51951.8346 & -26.00 & 0.23 & 52730.7218 & -30.40 & 0.45 & 54572.8004 & -8.27 & 0.45 \\
\hline 51952.8300 & -26.70 & 0.22 & 52731.7150 & -30.50 & 0.50 & 54625.5740 & -14.00 & 0.13 \\
\hline 51953.8449 & -27.37 & 0.18 & 52732.8830 & -30.54 & 0.54 & 54625.6043 & -14.11 & 0.12 \\
\hline 52015.7748 & -28.58 & 0.24 & 52733.7957 & -30.30 & 0.49 & 54628.5107 & -14.66 & 0.10 \\
\hline 52016.8033 & -28.21 & 0.15 & 52735.6860 & -29.25 & 0.46 & 54628.7443 & -14.58 & 0.05 \\
\hline 52017.7562 & -27.22 & 0.08 & 52736.7746 & -28.41 & 0.47 & 54629.5725 & -14.49 & 0.19 \\
\hline 52018.7724 & -26.36 & 0.13 & 52737.7340 & -28.57 & 0.60 & 54629.6498 & -14.32 & 0.12 \\
\hline 52019.7750 & -25.38 & 0.16 & 52738.7553 & -28.37 & 0.67 & 54630.5171 & -14.41 & 0.13 \\
\hline 52020.7769 & -24.35 & 0.10 & 52739.7448 & -27.68 & 0.51 & 54632.5266 & -14.15 & 0.25 \\
\hline 52021.7452 & -23.51 & 0.09 & 52740.8043 & -27.81 & 0.39 & 54670.5925 & -20.79 & 0.45 \\
\hline 52024.7130 & -23.23 & 0.21 & 52803.5603 & -21.56 & 0.84 & 54671.6170 & -20.17 & 0.60 \\
\hline 52025.7119 & -23.40 & 0.23 & 52804.4995 & -22.23 & 0.80 & 54672.5327 & -20.18 & 0.42 \\
\hline 52026.7477 & -23.58 & 0.21 & 52817.5699 & -24.40 & 0.60 & 54674.6597 & -20.54 & 0.61 \\
\hline 52027.7771 & -23.45 & 0.29 & 53072.8326 & -17.46 & 0.20 & 54677.5327 & -22.27 & 0.21 \\
\hline 52111.6771 & -11.26 & 0.19 & 53073.7938 & -17.43 & 0.24 & 54678.6760 & -22.59 & 0.21 \\
\hline & & & & & & 54679.6688 & -22.68 & 0.16 \\
\hline \multicolumn{9}{|c|}{ IRAS 19125+0343 } \\
\hline 51277.9046 & 67.62 & 0.34 & 52121.7094 & 75.54 & 0.71 & 53180.7526 & 80.94 & 0.09 \\
\hline 51281.9142 & 67.48 & 0.61 & 52123.7786 & 75.24 & 0.48 & 53182.8327 & 81.45 & 0.11 \\
\hline 51469.5423 & 52.73 & 0.26 & 52416.8105 & 55.29 & 0.13 & 53183.7827 & 82.19 & 0.74 \\
\hline 51654.8809 & 79.72 & 0.66 & 52417.8448 & 56.07 & 0.11 & 53184.7404 & 79.96 & 0.16 \\
\hline 51655.8857 & 79.70 & 0.71 & 52424.8240 & 55.49 & 0.48 & 53184.7839 & 79.62 & 0.22 \\
\hline 51659.9231 & 81.77 & 0.36 & 52468.8291 & 58.53 & 0.62 & 53190.8718 & 75.60 & 0.22 \\
\hline 51760.6545 & 71.90 & 0.52 & 52570.5382 & 66.61 & 0.31 & 53191.8179 & 75.50 & 0.38 \\
\hline 51762.6816 & 70.35 & 0.32 & 52571.5524 & 66.88 & 0.18 & 53224.7553 & 75.40 & 0.20 \\
\hline 51763.6553 & 69.59 & 0.20 & 52572.5529 & 66.49 & 0.54 & 53232.7510 & 74.68 & 0.45 \\
\hline 51768.6680 & 68.74 & 0.13 & 52573.5369 & 67.15 & 0.14 & 53271.6065 & 75.07 & 0.30 \\
\hline 51773.7340 & 68.30 & 0.43 & 52574.5574 & 68.15 & 0.08 & 53273.5852 & 73.95 & 0.16 \\
\hline 51817.5984 & 64.36 & 0.26 & 52575.5369 & 69.39 & 0.27 & 53275.5725 & 72.53 & 0.09 \\
\hline 51819.5323 & 65.19 & 0.29 & 52576.5504 & 70.35 & 0.20 & 542 & 72.98 & 0.08 \\
\hline 51820.5569 & 65.97 & 0.36 & 52577.5583 & 70.66 & 0.14 & 53280.5621 & 73.21 & 0.27 \\
\hline 51821.5251 & 65.14 & 0.46 & 52578.5386 & 70.56 & 0.32 & 53283.5685 & 72.39 & 0.25 \\
\hline 51822.5530 & 64.91 & 0.05 & 52579.5452 & 69.48 & 0.27 & 53477.8754 & 54.14 & 0.35 \\
\hline 51823.5479 & 64.74 & 0.07 & 52580.5299 & 67.78 & 0.51 & 53479.8841 & 54.14 & 0.69 \\
\hline 51824.5235 & 64.72 & 0.14 & 52581.5401 & 66.86 & 0.12 & 53481.8821 & 54.62 & 0.65 \\
\hline 51825.5270 & 65.30 & 0.13 & 52734.8964 & 77.60 & 0.12 & 53482.8847 & 54.82 & 0.45 \\
\hline 51826.5340 & 66.08 & 0.11 & 52735.8820 & 77.49 & 0.08 & 53483.8867 & 54.77 & 0.36 \\
\hline 51827.5488 & 66.27 & 0.15 & 52737.8768 & 76.76 & 0.08 & 53648.5544 & 71.51 & 0.54 \\
\hline 51828.5417 & 66.19 & 0.14 & 52738.9052 & 76.98 & 0.34 & 53651.5256 & 73.75 & 0.31 \\
\hline 52015.9054 & 58.46 & 0.64 & 52739.8834 & 77.10 & 0.28 & 53653.5139 & 75.62 & 0.72 \\
\hline 52018.8886 & 60.38 & 0.14 & 52740.8896 & 77.02 & 0.25 & 53654.5258 & 75.79 & 0.94 \\
\hline 52024.8528 & 60.05 & 0.36 & 52854.7053 & 64.85 & 0.07 & 53898.8262 & 71.10 & 0.68 \\
\hline 52025.8665 & 59.34 & 0.15 & 52857.6949 & 68.01 & 0.26 & 53901.8042 & 69.85 & 0.66 \\
\hline 52026.8543 & 60.98 & 0.68 & 52858.6964 & 69.00 & 0.28 & 53954.7359 & 61.06 & 0.62 \\
\hline 52114.6868 & 76.02 & 0.18 & 52866.6696 & 70.17 & 0.29 & 54302.7889 & 72.11 & 0.59 \\
\hline 52115.6880 & 75.35 & 0.22 & 52867.6726 & 68.24 & 0.36 & 54303.7651 & 72.13 & 0.37 \\
\hline \multirow[t]{2}{*}{52118.6850} & 75.39 & 0.31 & 53179.7887 & 79.78 & 0.25 & 54331.6422 & 73.60 & 0.82 \\
\hline & & & & & & 54666.8072 & 73.13 & 0.53 \\
\hline
\end{tabular}


H. Van Winckel et al.: Binarity of post-AGB stars, Online Material p 4

Table 4. continued.

\begin{tabular}{|c|c|c|c|c|c|c|c|c|}
\hline$\overline{J D}(24+)$ & $\overline{V(\mathrm{rad})}$ & $\overline{\sigma \sigma}$ & JD(24+) & $\overline{V(\mathrm{rad})}$ & $\overline{\sigma \sigma}$ & JD(24+) & $\overline{V(\mathrm{rad})}$ & $\overline{\sigma \sigma}$ \\
\hline \multicolumn{9}{|c|}{ IRAS $19157-0247$} \\
\hline 51278.9071 & 23.53 & 0.27 & 52416.8728 & 34.95 & 0.68 & 53272.5760 & 39.39 & 1.02 \\
\hline 51280.9177 & 21.31 & 0.60 & 52417.9179 & 37.25 & 0.92 & 53273.5944 & 37.06 & 1.10 \\
\hline 51469.5487 & 37.83 & 0.63 & 52571.5700 & 23.03 & 0.85 & 53274.5934 & 38.01 & 0.19 \\
\hline 51471.5432 & 38.91 & 0.33 & 52572.5797 & 26.16 & 0.94 & 53275.5991 & 38.67 & 0.24 \\
\hline 51507.5279 & 25.58 & 0.90 & 52574.5789 & 29.83 & 0.42 & 53279.5319 & 42.27 & 0.20 \\
\hline 51652.9129 & 26.58 & 0.43 & 52575.5604 & 30.82 & 0.72 & 53281.5973 & 42.64 & 1.03 \\
\hline 51654.8925 & 27.64 & 0.40 & 52576.5738 & 30.20 & 0.74 & 53475.9058 & 29.89 & 0.50 \\
\hline 51760.6956 & 26.46 & 0.36 & 52578.5621 & 27.82 & 0.27 & 53476.9032 & 31.35 & 1.13 \\
\hline 51761.6696 & 28.09 & 0.31 & 52579.5684 & 26.96 & 0.50 & 53477.9035 & 34.83 & 0.00 \\
\hline 51762.7170 & 30.41 & 0.17 & 52580.5533 & 25.16 & 1.05 & 53478.9114 & 36.92 & 0.32 \\
\hline 51763.6769 & 32.51 & 0.26 & 52581.5633 & 22.01 & 1.40 & 53479.8996 & 38.47 & 0.12 \\
\hline 51765.6332 & 34.48 & 0.23 & 52728.8557 & 20.67 & 0.58 & 53481.9049 & 38.40 & 0.37 \\
\hline 51766.6211 & 35.12 & 0.27 & 52734.8735 & 33.11 & 0.26 & 53482.9121 & 39.90 & 0.86 \\
\hline 51769.7570 & 26.37 & 0.93 & 52735.8335 & 33.70 & 0.33 & 53483.9209 & 40.61 & 1.12 \\
\hline 51772.5723 & 24.10 & 0.17 & 52736.8830 & 33.63 & 0.23 & 53651.5721 & 27.45 & 0.73 \\
\hline 51816.5981 & 39.53 & 0.65 & 52737.9002 & 32.41 & 0.20 & 53652.5030 & 26.45 & 1.35 \\
\hline 51817.6263 & 39.84 & 0.28 & 52738.8819 & 32.63 & 0.45 & 53653.5579 & 26.00 & 0.80 \\
\hline 51819.5685 & 41.45 & 0.53 & 52739.8598 & 32.19 & 0.50 & 53809.8992 & 25.62 & 0.62 \\
\hline 51820.5746 & 43.44 & 0.46 & 52740.9131 & 31 & 0.49 & 02.8038 & 46 & 0.19 \\
\hline 5182 & 44 & 0.66 & 52854.7293 & 33.04 & 0.50 & 53903. & 30.22 & 1.23 \\
\hline 51822.5745 & 43.81 & 0.39 & 52855.7233 & 29.67 & 0.35 & 53951 & 35.49 & 1.18 \\
\hline 51823.5724 & 43.79 & 0.47 & 52857.7190 & 27.54 & 0.52 & 53954 & 33.80 & 0.00 \\
\hline 51824.5479 & 41.91 & 0.81 & 52859.7232 & 27.54 & 0.72 & 53956.7439 & 33.99 & 1.09 \\
\hline 51825.5514 & 38.89 & 1.16 & 52861.7367 & 27.65 & 0.09 & 53960.7338 & 41.74 & 1.15 \\
\hline 51826.5596 & 36.03 & 1.04 & 52862.7190 & 26.11 & 0.25 & 54303.7769 & 33.05 & 0.83 \\
\hline 51827.5749 & 36.71 & 0.38 & 52865.7062 & 26.99 & 0.33 & 54305.8161 & 37.83 & 0.54 \\
\hline 51828.5661 & 37.19 & 0.39 & 52867.6963 & 33.14 & 0.23 & 54308.7831 & 44.33 & 0.64 \\
\hline 51829.5199 & 38.42 & 0.18 & 53179.8589 & 25.49 & 0.20 & 54581.9151 & 44.84 & 1.11 \\
\hline 51829.5403 & 38.17 & 0.27 & 53179.8946 & 26.30 & 1.00 & 54625.8859 & 28.83 & 1.48 \\
\hline 52020.8803 & 36.02 & 0.43 & 53184.8284 & 25.87 & 1.37 & 54626.8699 & 28.58 & 0.61 \\
\hline 52025.8955 & 27.45 & 0.36 & 53188.8789 & 25.64 & 0.60 & 54628.7670 & 27.76 & 0.71 \\
\hline 52026.8832 & 28.65 & 0.32 & 53190.8982 & 24.37 & 1.44 & 54630.7473 & 27.48 & 0.32 \\
\hline 52027.8899 & 29.46 & 0.19 & 53191.8399 & 24.05 & 0.75 & 54630.7917 & 28.22 & 1.33 \\
\hline 52114.8391 & 29.58 & 1.12 & 53224.7904 & 33.36 & 0.54 & 54632.7171 & 28.95 & 1.20 \\
\hline 52116.8258 & 28.42 & 1.29 & 53232.7181 & 34.83 & 0.28 & 54666.8228 & 33.33 & 0.37 \\
\hline 52227.5114 & 29.59 & 0.32 & 53270.5775 & 38.37 & 0.72 & 54672.7039 & 32.89 & 0.90 \\
\hline 52227.5322 & 29.76 & 0.21 & 53271.6303 & 39.54 & 0.82 & 54675.7832 & 37.97 & 1.40 \\
\hline
\end{tabular}


H. Van Winckel et al.: Binarity of post-AGB stars, Online Material p 5

Table 5. Individual photometric datapoints of the programme stars obtained at SAAO.

\begin{tabular}{|c|c|c|c|c|c|c|c|c|c|c|c|}
\hline HJD & 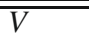 & 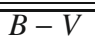 & $\overline{U-B}$ & $\overline{V V-R}$ & $\overline{\overline{V-I}}$ & HJD & $\bar{V}$ & $B-V$ & $U-B$ & $V-R$ & $V-I$ \\
\hline \multicolumn{12}{|c|}{ IRAS 08544-4431 } \\
\hline 2450858.47530 & 9.149 & 1.553 & 1.180 & 0.995 & 2.029 & 2451581.33470 & 9.163 & 1.580 & 1.191 & 0.992 & 2.042 \\
\hline 2450866.39190 & 9.164 & 1.555 & 1.167 & 0.993 & 2.025 & 2451582.39140 & 9.169 & 1.583 & 1.188 & 0.997 & 2.046 \\
\hline 2450867.38350 & 9.156 & 1.547 & 1.169 & 0.990 & 2.018 & 2451608.44360 & 9.035 & 1.511 & 1.148 & 0.961 & 1.982 \\
\hline 2450868.39470 & 9.168 & 1.546 & 1.154 & 0.990 & 2.017 & 2451609.37170 & 9.017 & 1.516 & 1.127 & 0.965 & 1.993 \\
\hline 2450869.41250 & 9.153 & 1.545 & 1.157 & 0.994 & 2.026 & 2451625.28560 & 9.071 & 1.547 & 1.176 & 0.977 & 2.014 \\
\hline 2450871.39890 & 9.151 & 1.540 & 1.151 & 0.994 & 2.020 & 2451626.29140 & 9.072 & 1.547 & 1.173 & 0.977 & 2.017 \\
\hline 2450872.40870 & 9.169 & 1.541 & 1.158 & 0.999 & 2.011 & 2451663.23130 & 9.133 & 1.548 & 1.162 & 0.979 & 2.036 \\
\hline 2450874.38380 & 9.158 & 1.536 & 1.156 & 0.992 & 2.014 & 2451666.22130 & 9.106 & 1.538 & 1.155 & 0.972 & 2.014 \\
\hline 2450879.39550 & 9.163 & 1.532 & 1.160 & 0.984 & 2.005 & 2451929.49208 & 9.142 & 1.548 & 1.162 & 0.995 & 2.021 \\
\hline 2450883.34250 & 9.140 & 1.539 & 1.161 & 0.983 & 2.010 & 2451930.48697 & 9.149 & 1.547 & 1.180 & 0.996 & 2.026 \\
\hline 2450884.46330 & 9.167 & 1.537 & 1.149 & 0.997 & 2.014 & 2451931.49118 & 9.163 & 1.547 & 1.189 & 1.001 & 2.036 \\
\hline 2450885.37300 & 9.133 & 1.538 & 1.193 & 0.990 & 2.006 & 2451936.49577 & 9.180 & 1.555 & 1.190 & 1.000 & 2.028 \\
\hline 2450886.41580 & 9.127 & 1.536 & 1.190 & 0.984 & 2.013 & 2451939.53417 & 9.174 & 1.566 & 1.326 & 1.005 & 2.043 \\
\hline 2450887.44010 & 9.123 & 1.544 & 1.163 & 0.992 & 2.017 & 2451941.48938 & 9.168 & 1.561 & 1.196 & 1.006 & 2.037 \\
\hline 2450889.44560 & 9.122 & 1.544 & 1.165 & 0.996 & 2.021 & 2451944.48373 & 9.167 & 1.550 & 1.158 & 1.005 & 2.039 \\
\hline 2450918.28290 & 9.144 & 1.555 & 1.178 & 0.989 & 2.023 & 2451945.50376 & 9.175 & 1.555 & 1.150 & 1.002 & 2.038 \\
\hline 2450919.28720 & 9.148 & 1.552 & 1.175 & 0.994 & 2.027 & 2451956.47669 & 9.129 & 1.557 & 1.188 & 1.001 & 2.038 \\
\hline 2450929.29080 & 9.180 & 1.541 & 1.144 & 0.996 & 2.022 & 2451957.45032 & 9.125 & 1.549 & 1.170 & 1.002 & 2.032 \\
\hline 2450931.32500 & 9.173 & 1.542 & 1.149 & 0.994 & 2.023 & 2451960.40009 & 9.122 & 1.541 & 1.153 & 1.001 & 2.031 \\
\hline 2450937.26810 & 9.160 & 1.544 & 1.143 & 0.997 & 2.033 & 2451961.44573 & 9.112 & 1.543 & 1.157 & 0.993 & 2.022 \\
\hline 2450948.26710 & 9.116 & 1.540 & 1.152 & 0.989 & 2.017 & 2451962.44569 & 9.112 & 1.540 & 1.154 & 0.990 & 2.020 \\
\hline 2450957.21450 & 9.110 & 1.522 & 1.136 & 0.984 & 2.010 & 245196 & 9.106 & 1.531 & 1.120 & 0.984 & 2.019 \\
\hline 2450959.2 & 9.114 & 1.523 & 1.145 & 0.982 & 2.011 & 24519 & 9.113 & 1.544 & 1.149 & 0.988 & 2.023 \\
\hline 2451205.4 & 9.143 & 1.5 & 1.165 & 0.990 & 2.030 & 245 & 10 & & 60 & 0.996 & 2.018 \\
\hline 2451209.41850 & 9.132 & 1.554 & 1.161 & 0.999 & 2.034 & 245196 & 9.098 & 37 & 63 & 0.984 & 2.016 \\
\hline 2451210.39440 & 9.139 & 1.553 & 1.196 & 0.995 & 2.031 & 245197 & 9.098 & 1.5 & 18 & 0.986 & 2.006 \\
\hline 2451215.42880 & 9.155 & 1.549 & 1.196 & 0.996 & 2.033 & $24519^{7}$ & 9.082 & 3 & 3 & 0.991 & 2.018 \\
\hline 2451216.40510 & 9.148 & 1.555 & 1.179 & 0.994 & 2.026 & 2451976.44461 & 9.082 & 1.549 & 1.462 & 0.985 & 2.014 \\
\hline 2451228.38630 & 9.148 & 1.559 & 1.190 & 0.991 & 2.028 & 2451981.36532 & 9.087 & 1.538 & 1.289 & 0.988 & 2.016 \\
\hline 2451230.37560 & 9.138 & 1.553 & 1.175 & 0.988 & 2.024 & 2451982.36317 & 9.080 & 1.530 & 1.200 & 0.994 & 2.021 \\
\hline 2451231.37210 & 9.142 & 1.546 & 1.178 & 0.992 & 2.027 & 2451988.35682 & 9.101 & 1.529 & 1.144 & 0.990 & 2.024 \\
\hline 2451232.36380 & 9.145 & 1.549 & 1.193 & 0.990 & 2.026 & 2452002.34988 & 9.093 & 1.537 & 1.142 & 0.994 & 2.022 \\
\hline 2451233.36640 & 9.145 & 1.549 & 1.181 & 0.991 & 2.023 & 2452003 & 9.093 & 1.529 & 1.139 & 0.991 & 2.028 \\
\hline 2451234.34890 & 9.146 & 1.543 & 1.177 & 0.993 & 2.030 & 24520 & 9.081 & 1.537 & 1.173 & 0.988 & 2.020 \\
\hline 235. & .143 & 1.541 & 1.188 & 0.997 & 2.028 & 245 & 9.086 & 1.536 & 1.183 & 0.986 & 2.024 \\
\hline 2451236.34840 & 9.136 & 1.545 & 1.175 & 0.987 & 2.016 & 245 & 13 & 33 & 50 & 0.992 & 2.027 \\
\hline 2451238.33990 & 9.128 & 1.537 & 1.161 & 0.993 & 2.021 & 245202 & 22 & 32 & 45 & 0.993 & 2.027 \\
\hline 239.3 & 9.1 & 1.5 & 1.200 & 35 & 2.016 & 94 & 28 & 28 & 39 & 90 & 2.021 \\
\hline 240 & 9.1 & 1.5 & 11 & 22 & 2.020 & 24 & 9. & 77 & 3 & 0.980 & 2.023 \\
\hline 2451298.2 & .192 & 1.575 & 1.178 & 1.007 & 2.056 & 245 & 9.142 & 44 & 9 & 0.994 & 2.027 \\
\hline 2451300.23330 & 9.192 & 1.564 & 1.226 & 1.006 & 2.053 & 24520 & 9.141 & 1.543 & 1.141 & 0.998 & 2.032 \\
\hline 2451303.24530 & 9.170 & 1.557 & 1.182 & 0.997 & 2.040 & 2452043.23925 & 9.137 & 1.541 & 1.144 & 0.984 & 2.015 \\
\hline 2451309.23600 & 9.154 & 1.548 & 1.169 & 0.999 & 2.040 & 2452045.25507 & 9.130 & 1.545 & 1.142 & 0.989 & 2.033 \\
\hline 2451576.40290 & 9.179 & 1.589 & 1.203 & 0.996 & 2.050 & 2452057.26545 & 9.080 & 1.529 & 1.117 & 0.986 & 2.020 \\
\hline 2451579.40000 & 9.168 & 1.580 & 1.213 & 0.993 & 2.049 & 2452060.29439 & 9.062 & 1.533 & 1.140 & 0.990 & 2.027 \\
\hline 2451580.35280 & 9.167 & 1.582 & 1.183 & 0.993 & 2.046 & & & & & & \\
\hline \multicolumn{12}{|c|}{ IRAS 12222-4652 } \\
\hline 2451 & 977 & 426 & 0.345 & 265 & 0.528 & 2452428 & 7.936 & 0.423 & 0.335 & 0.255 & 0.523 \\
\hline 2451626.5038 & 8.005 & 0.425 & 0.334 & 0.274 & 0.542 & 2452429.2922 & 7.929 & 0.421 & 0.329 & 0.255 & 0.519 \\
\hline 2451663.3917 & 7.972 & 0.423 & 0.337 & 0.259 & 0.519 & 2452430.2371 & 7.935 & 0.422 & 0.345 & 0.239 & 0.510 \\
\hline 2451688.3192 & 7.937 & 0.408 & 0.342 & 0.248 & 0.507 & 2452431.2499 & 7.937 & 0.414 & 0.337 & 0.247 & 0.520 \\
\hline 2451691.4224 & 7.957 & 0.421 & 0.330 & 0.257 & 0.516 & 2452440.2451 & 7.889 & 0.397 & 0.337 & 0.213 & 0.485 \\
\hline 2451692.3194 & 7.965 & 0.417 & 0.342 & 0.256 & 0.525 & 2452441.2684 & 7.869 & 0.404 & 0.313 & 0.239 & 0.497 \\
\hline 2451712.2471 & 7.917 & 0.408 & 0.337 & 0.250 & 0.509 & 2452445.2723 & 7.889 & 0.404 & 0.332 & 0.243 & 0.491 \\
\hline 2451976.5085 & 8.004 & 0.433 & 0.369 & 0.267 & 0.544 & 2452450.2713 & 7.917 & 0.405 & 0.334 & 0.248 & 0.508 \\
\hline 2451977.5076 & 8.011 & 0.441 & 0.364 & 0.272 & 0.540 & 2452458.2337 & 7.966 & 0.417 & 0.349 & 0.250 & 0.531 \\
\hline 2451978.4812 & 8.005 & 0.425 & 0.358 & 0.271 & 0.546 & 2452684.5593 & 7.880 & 0.402 & 0.331 & 0.245 & 0.492 \\
\hline 2451980.5089 & 8.004 & 0.434 & 0.324 & 0.271 & 0.541 & 2452686.5516 & 7.876 & 0.402 & 0.331 & 0.239 & 0.495 \\
\hline 2451981.5092 & 8.002 & 0.433 & 0.364 & 0.269 & 0.536 & 2452687.5810 & 7.881 & 0.403 & 0.328 & 0.244 & 0.498 \\
\hline 2451982.4785 & 8.006 & 0.433 & 0.359 & 0.268 & 0.547 & 2452690.5702 & 7.892 & 0.402 & 0.331 & 0.242 & 0.493 \\
\hline 2451988.5325 & 7.983 & 0.432 & 0.340 & 0.261 & 0.523 & 2452691.5556 & 7.917 & 0.408 & 0.344 & 0.243 & 0.492 \\
\hline 2452017.3946 & 7.922 & 0.398 & 0.335 & 0.248 & 0.504 & 2452692.5686 & 7.936 & 0.411 & 0.344 & 0.251 & 0.506 \\
\hline 2452017.4016 & 7.919 & 0.399 & 0.329 & 0.250 & 0.508 & 2452693.5684 & 7.944 & 0.413 & 0.343 & 0.250 & 0.508 \\
\hline 2452018.5058 & 7.921 & 0.402 & 0.338 & 0.243 & 0.502 & 2452701.5593 & 7.994 & 0.431 & 0.352 & 0.255 & 0.522 \\
\hline
\end{tabular}


H. Van Winckel et al.: Binarity of post-AGB stars, Online Material p 6

Table 5. continued.

\begin{tabular}{|c|c|c|c|c|c|c|c|c|c|c|c|}
\hline$\overline{\mathrm{HJD}}$ & $\overline{\bar{V}}$ & $\overline{B-V}$ & $\overline{\overline{U-B}}$ & $\overline{V V-R}$ & $\overline{V-I}$ & "HJD & $\overline{\bar{V}}$ & $\overline{\bar{B} B-V}$ & $\overline{\overline{U-B}}$ & $\overline{V V-R}$ & $\overline{V-I}$ \\
\hline 2452019.3085 & 7.915 & 0.403 & 0.345 & 0.246 & 0.501 & 2452705.5750 & 8.009 & 0.444 & 0.348 & 0.266 & 0.540 \\
\hline 2452022.3214 & 7.941 & 0.414 & 0.339 & 0.250 & 0.509 & 2452706.5443 & 8.022 & 0.453 & 0.347 & 0.270 & 0.541 \\
\hline 2452025.4453 & 7.961 & 0.415 & 0.338 & 0.257 & 0.519 & 2452707.5710 & 8.024 & 0.456 & 0.346 & 0.276 & 0.545 \\
\hline 2452029.3838 & 7.985 & 0.433 & 0.350 & 0.264 & 0.533 & 2452710.5422 & 8.017 & 0.456 & 0.354 & 0.277 & 0.559 \\
\hline 2452030.2856 & 7.979 & 0.435 & 0.343 & 0.258 & 0.531 & 245272 & 7.891 & 0.407 & 0.320 & 0.242 & 0.505 \\
\hline 2452033.3263 & 7.970 & 0.431 & 0.341 & 0.262 & 0.533 & 2452 & 7.876 & 0.404 & 0.313 & 0.241 & 0.500 \\
\hline 2452033.3603 & 7.983 & 0.431 & 40 & 0.260 & 0.533 & 245272 & 7.866 & 0.392 & 0.318 & 0.239 & 0.495 \\
\hline 2452037.3227 & 7.972 & 0.418 & 0.335 & 0.257 & 0.531 & 2452728.5041 & 7.861 & 0.395 & 0.311 & 0.239 & 0.494 \\
\hline 2452038.3380 & 7.969 & 0.415 & 0.329 & 0.259 & 0.529 & 2452729.4815 & 7.852 & 0.386 & 0.312 & 0.237 & 0.495 \\
\hline 2452040.3509 & 7.958 & 0.418 & 0.332 & 0.260 & 0.529 & 2452730.4904 & 7.837 & 0.395 & 0.301 & 0.236 & 0.493 \\
\hline 2452041.3004 & 7.964 & 0.421 & 0.326 & 0.261 & 0.529 & 2452731.5323 & 7.858 & 0.389 & 0.299 & 0.240 & 0.499 \\
\hline 2452041.3152 & 7.964 & 0.420 & 0.331 & 0.259 & 0.524 & 2452732.4831 & 7.839 & 0.393 & 0.294 & 0.238 & 0.495 \\
\hline 2452042.2969 & 7.961 & 0.414 & 0.332 & 0.262 & 0.530 & 2452733.4773 & 7.840 & 0.395 & 0.301 & 0.231 & 0.489 \\
\hline 2452042.3178 & 7.957 & 0.426 & 0.329 & 0.261 & 0.523 & 2452734.4769 & 7.834 & 0.391 & 0.299 & 0.236 & 0.491 \\
\hline 2452043.2893 & 7.952 & 0.423 & 0.324 & 0.256 & 0.523 & 2452753.4278 & 7.965 & 0.421 & 0.333 & 0.252 & 0.521 \\
\hline 2452045.2852 & 7.950 & 0.424 & 0.335 & 0.260 & 0.533 & 2452754.4274 & 7.967 & 0.421 & 0.338 & 0.251 & 0.518 \\
\hline 2452057.2957 & 7.917 & 0.404 & 0.319 & 0.244 & 0.506 & 2452 & 7.972 & 0.422 & 0.339 & 0.254 & 0.524 \\
\hline 2452057.3704 & 7.923 & 0.406 & 0.308 & 0.253 & 0.517 & 245 & 7.968 & 0.424 & 0.340 & 0.253 & 0.520 \\
\hline 2452060 & 7.922 & 0.410 & 0.330 & 0.251 & 0.512 & 24 & 8.000 & 0.429 & 0.344 & 0.257 & 0.531 \\
\hline 2452061. & 7.902 & 0.415 & 0.337 & 0.248 & 0.509 & 2452 & 8.005 & 0.440 & 0.336 & 0.260 & 0.533 \\
\hline 2452072.3048 & 003 & 0.436 & 0.328 & 0.276 & 0.545 & 2452 & 8.013 & 0.436 & 0.350 & 0.265 & 0.537 \\
\hline 2452073.2156 & 7.974 & 0.418 & 0.343 & 0.258 & 0.526 & 2452 & 7.999 & 0.431 & 0.338 & 0.268 & 0.536 \\
\hline 2452073.2555 & 7.965 & 0.419 & 0.351 & 0.259 & 0.526 & 2452 & 8.032 & 0.452 & 0.359 & 0.035 & 0.488 \\
\hline 2452074.2161 & 7.954 & 0.423 & 0.334 & 0.255 & 0.526 & 2452 & 7.966 & 0.414 & 0.308 & 0.253 & 0.521 \\
\hline 2452075.2183 & 7.965 & 0.416 & 0.342 & 0.252 & 0.531 & 2452783.3404 & 7.957 & 0.414 & 0.328 & 0.257 & 0.519 \\
\hline 2452075.2587 & 7.965 & 0.404 & 0.338 & 0.274 & 0.540 & 2452789.3391 & 7.882 & 0.394 & 0.323 & 0.241 & 0.493 \\
\hline 2452076.2181 & 7.958 & 0.419 & 0.343 & 0.251 & 0.528 & 2453 & 7.937 & 0.413 & 0.317 & 0.248 & 0.507 \\
\hline 2452076.2558 & 7.957 & 0.411 & 0.339 & 0.264 & 0.533 & 2453 & 7.961 & 0.419 & 0.316 & 0.252 & 0.511 \\
\hline 2452080.2331 & 7.978 & 0.433 & 0.351 & 0.267 & 0.535 & 245 & 7.929 & 0.401 & 0.329 & 0.253 & 0.507 \\
\hline 24520 & 7.981 & 0.425 & 0.345 & 0.273 & 0.543 & & 7.915 & 0.401 & 0.313 & 0.245 & 0.502 \\
\hline 245208 & 7.996 & 0.427 & 0.346 & 0.260 & 0.536 & 24 & 7.921 & 0.399 & 0.287 & 0.238 & 0.489 \\
\hline 24520 & 7.979 & 0.428 & 0.343 & 0.264 & 0.536 & 24 & 7.895 & 0.402 & 0.323 & 0.241 & 0.500 \\
\hline 24520 & 7.975 & 0.443 & 0.347 & 0.256 & 0.542 & 245 & 7.906 & 0.404 & 0.338 & 0.246 & 0.499 \\
\hline 2452 & .986 & 0.435 & 0.355 & 0.262 & 0.544 & 24 & 7.922 & 0.417 & 0.338 & 0.239 & 0.503 \\
\hline 24520 & .979 & 0.434 & 0.344 & 0.267 & 0.538 & 24 & 7.927 & 0.420 & 0.335 & 0.249 & 0.511 \\
\hline 2452093 & .971 & 0.429 & 0.342 & 0.265 & 0.541 & 245 & 7.929 & 0.422 & 0.333 & 0.246 & 0.511 \\
\hline 2452112.2266 & .946 & 0.401 & 0.335 & 0.251 & 0.519 & 245 & 7.937 & 0.414 & 0.346 & 0.255 & 0.512 \\
\hline 2452319.5708 & 7.939 & 0.403 & 0.331 & 0.248 & 0.503 & 2453 & 7.989 & 0.440 & 0.341 & 0.258 & 0.534 \\
\hline 2452320.5606 & 7.950 & 0.413 & 0.337 & 0.242 & 0.497 & 2453 & 7.995 & 0.431 & 0.339 & 0.262 & 0.528 \\
\hline 2452321.5606 & 7.949 & 0.413 & 0.338 & 0.241 & 0.497 & 9.4551 & 7.860 & 0.393 & 0.329 & 0.230 & 0.482 \\
\hline 2452322.5773 & 7.955 & 0.414 & 0.333 & 0.255 & 0.512 & 2453 & 7.886 & 0.392 & 0.330 & 0.237 & 0.489 \\
\hline 2452324.5259 & 7.962 & 0.410 & 0.346 & 0.260 & 0.520 & 2453 & 7.904 & 0.394 & 0.319 & 0.246 & 0.492 \\
\hline 2452324.6051 & 7.956 & 0.414 & 0.340 & 0.247 & 0.509 & 2453 & 7.906 & 0.400 & 0.328 & 0.242 & 0.494 \\
\hline 2452327.5389 & 7.956 & 0.420 & 0.335 & 0.261 & 0.518 & 245 & 7.905 & 0.395 & 0.331 & 0.251 & 0.504 \\
\hline 2452336.5080 & 7.931 & 0.415 & 0.347 & 0.244 & 0.506 & 24 & 7.948 & 0.408 & 0.333 & 0.241 & 0.501 \\
\hline 24523 & 7.965 & 0. & 0. & 0.267 & 0.523 & 2 & 7.958 & 07 & 29 & 0.248 & 0.505 \\
\hline 24523 & 7.954 & 0. & 40 & 0.259 & 0.520 & 24 & 8.005 & 0.427 & 0.342 & 0.264 & 0.530 \\
\hline 2452 & .955 & 0.425 & 55 & 0.255 & 0.518 & 24 & 8.022 & 0.455 & 0.359 & 0.281 & 0.552 \\
\hline 2452 & 964 & 0.426 & 53 & 0.258 & 0.516 & 24 & 8.048 & 0.458 & 0.367 & 0.277 & 0.550 \\
\hline 2452350.5134 & 961 & 0.426 & 0.346 & 0.258 & 0.518 & 2453 & 8.037 & 0.462 & 0.351 & 0.271 & 0.549 \\
\hline 2452352.4633 & .964 & 0.427 & 0.353 & 0.256 & 0.521 & 24531 & 8.036 & 0.465 & 0.350 & 0.275 & 0.547 \\
\hline 2452356.4939 & 7.945 & 0.419 & 0.348 & 0.264 & 0.518 & 2453144.2756 & 8.021 & 0.461 & 0.347 & 0.276 & 0.556 \\
\hline 2452357.5297 & 7.946 & 0.419 & 0.349 & 0.250 & 0.513 & 2453146.3328 & 8.008 & 0.453 & 0.343 & 0.270 & 0.551 \\
\hline 2452376.3944 & 7.989 & 0.418 & 0.347 & 0.285 & 0.550 & 24531 & 8.005 & 0.455 & 0.335 & 0.269 & 0.544 \\
\hline 2452377.3793 & 7.954 & 0.431 & 0.356 & 0.264 & 0.529 & 2453152.2814 & 7.979 & 0.443 & 0.331 & 0.256 & 0.532 \\
\hline 2452381.4302 & 7.952 & 0.418 & 0.339 & 0.254 & 0.519 & 2453156.2780 & 7.924 & 0.416 & 0.306 & 0.248 & 0.511 \\
\hline 2452383.3994 & 7.947 & 0.417 & 0.340 & 0.261 & 0.523 & 2453158.3357 & 7.922 & 0.405 & 0.305 & 0.243 & 0.504 \\
\hline 2452384.3629 & 7.940 & 0.407 & 0.347 & 0.250 & 0.517 & 2453173.2689 & 7.865 & 0.385 & 0.315 & 0.231 & 0.480 \\
\hline 2452403.4261 & 7.979 & 0.420 & 0.349 & 0.254 & 0.520 & 2453174.2657 & 7.870 & 0.386 & 0.318 & 0.232 & 0.480 \\
\hline 2452404.3022 & 8.002 & 0.426 & 0.347 & 0.259 & 0.534 & 2453178.2723 & 7.902 & 0.403 & 0.319 & 0.247 & 0.506 \\
\hline 2452405.3038 & 7.985 & 0.432 & 0.356 & 0.257 & 0.519 & 2453180.2890 & 7.920 & 0.410 & 0.325 & 0.249 & 0.505 \\
\hline 2452408.3870 & 7.993 & 0.434 & 0.338 & 0.264 & 0.542 & 2453181.2758 & 7.924 & 0.416 & 0.328 & 0.249 & 0.506 \\
\hline 2452410.3089 & 7.995 & 0.445 & 0.362 & 0.262 & 0.538 & 2453184.2745 & 7.953 & 0.426 & 0.316 & 0.250 & 0.516 \\
\hline
\end{tabular}


Table 5. continued.

\begin{tabular}{|c|c|c|c|c|c|c|c|c|c|c|c|}
\hline$\overline{\mathrm{HJD}}$ & $\overline{\bar{V}}$ & $\overline{B B-V}$ & $\overline{U U-B}$ & $\overline{V V-R}$ & $\overline{\overline{V-I}}$ & 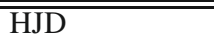 & $\overline{\bar{V}}$ & $\overline{B B-V}$ & $\overline{U U-B}$ & $\overline{V V-R}$ & $\overline{\overline{V-I}}$ \\
\hline 2452412.2881 & 7.999 & 0.446 & 0.355 & 0.265 & 0.540 & 2453185.2637 & 7.980 & 0.412 & 0.334 & 0.265 & 0.530 \\
\hline 2452413.3215 & 8.013 & 0.450 & 0.366 & 0.243 & 0.516 & 2453193.2971 & 8.008 & 0.431 & 0.338 & 0.259 & 0.530 \\
\hline 2452414.2939 & 7.987 & 0.435 & 0.346 & 0.261 & 0.538 & 2453194.2470 & 8.009 & 0.445 & 0.326 & 0.260 & 0.530 \\
\hline 2452426.2840 & 7.961 & 0.425 & 0.343 & 0.266 & 0.536 & 2453195.2581 & 8.017 & 0.441 & 0.322 & 0.264 & 0.536 \\
\hline \multicolumn{12}{|c|}{ IRAS 15469-5311 } \\
\hline 2448803.3438 & 10.518 & 1.428 & 1.064 & 0.890 & 1.823 & 2449213.2441 & 10.572 & 1.434 & 1.058 & 0.897 & 1.824 \\
\hline 2448829.3045 & 10.589 & 1.446 & 1.048 & 0.895 & 1.832 & 2449434.5918 & 10.567 & 1.447 & 1.072 & 0.902 & 1.840 \\
\hline 2448834.3133 & 10.580 & 1.442 & 1.065 & 0.888 & 1.835 & 2449438.6216 & 10.596 & 1.436 & 0.938 & 0.892 & 1.816 \\
\hline 2448844.2302 & 10.573 & 1.444 & 1.09 & 0.898 & 1.838 & 2449442.6260 & 10.622 & 1.468 & 0.973 & 0.904 & 1.853 \\
\hline 2449091.5265 & 10.540 & 1.432 & 1.037 & 0.888 & 1.815 & 2449485.4741 & 10.532 & 1.438 & 1.050 & 0.891 & 1.820 \\
\hline 2449093.5068 & 10.569 & 1.441 & 1.046 & 0.898 & 1.830 & 2449489.4590 & 10.493 & 1.428 & 1.064 & 0.884 & 1.817 \\
\hline 2449098.5501 & 10.578 & 1.450 & 1.043 & 0.895 & 1.826 & 2449490.4805 & 10.520 & 1.448 & 1.076 & 0.897 & 1.829 \\
\hline 2449102.6072 & 10.616 & 1.457 & 1.070 & 0.905 & 1.832 & 2449495.4346 & 10.503 & 1.426 & 1.036 & 0.895 & 1.821 \\
\hline 2449104.5423 & 10.593 & 1.449 & 1.040 & 0.899 & 1.828 & 2449498.5151 & 10.507 & 1.445 & 1.066 & 0.890 & 1.824 \\
\hline 2449107.5335 & 10.607 & 1.456 & 1.050 & 0.901 & 1.836 & 2449503.4702 & 10.525 & 1.432 & 1.080 & 0.892 & 1.824 \\
\hline 2449112.4527 & 10.581 & 1.459 & 1.009 & 0.896 & 1.829 & 2449510.4556 & 10.550 & 1.428 & 1.042 & 0.887 & 1.807 \\
\hline 2449115.4539 & 10.585 & 1.437 & 1.074 & 0.914 & 1.831 & 2449513.4326 & 10.565 & 1.433 & 1.050 & 0.892 & 1.822 \\
\hline 2449120.4480 & 10.555 & 1.437 & 1.045 & 0.897 & 1.827 & 2449515.3564 & 10.562 & 1.437 & 1.062 & 0.893 & 1.825 \\
\hline 2449124.5799 & 10.529 & 1.436 & 1.031 & 0.890 & 1.817 & 2449518.4131 & 10.556 & 1.441 & 1.058 & 0.896 & 1.820 \\
\hline 2449126.4214 & 10.527 & 1.438 & 1.036 & 0.890 & 1.817 & 2449534.4077 & 10.573 & 1.440 & 1.101 & 0.895 & 1.819 \\
\hline 2449128.4615 & 10.537 & 1.425 & 1.045 & 0.894 & 1.819 & 2449537.3081 & 10.553 & 1.434 & 1.056 & 0.890 & 1.810 \\
\hline 2449125.5241 & 10.538 & 1.431 & 1.025 & 0.891 & 1.819 & 2449539.3550 & 10.545 & 1.430 & 1.056 & 0.890 & 1.814 \\
\hline 2449138.3878 & 10.582 & 1.446 & 1.04 & 0.895 & 1.818 & 2449544.3740 & 10.532 & 1.433 & 1.060 & 0.892 & 1.808 \\
\hline 2449142.3606 & 10.573 & 1.456 & 1.061 & 0.886 & 1.821 & 2449562.2744 & 10.530 & 1.424 & 1.053 & 0.885 & 1.814 \\
\hline 2449146.4118 & 10.571 & 1.449 & 1.079 & 0.899 & 1.833 & 2449566.2617 & 10.543 & 1.438 & 1.062 & 0.887 & 1.816 \\
\hline 2449147.3900 & 10.563 & 1.445 & 1.085 & 0.895 & 1.830 & 2449574.2617 & 10.605 & 1.435 & 1.034 & 0.885 & 1.792 \\
\hline 2449156.4061 & 10.525 & 1.420 & 1.030 & 0.887 & 1.804 & 2449582.2446 & 10.571 & 1.457 & 1.085 & 0.900 & 1.842 \\
\hline 2449168.3833 & 10.543 & 1.442 & 1.075 & 0.881 & 1.801 & 2451626.5864 & 10.516 & 1.434 & 0.981 & 0.889 & 1.826 \\
\hline 2449172.3808 & 10.567 & 1.436 & 1.056 & 0.891 & 1.810 & 2451712.3599 & 10.527 & 1.479 & 1.131 & 0.894 & 1.808 \\
\hline 2449209.3057 & 10.522 & 1.430 & 1.028 & 0.888 & 1.807 & & & & & & \\
\hline \multicolumn{12}{|c|}{$\overline{\text { IRAS } 19125+0343}$} \\
\hline 2449485.5996 & 10.136 & 1.003 & 0.644 & 0.675 & 1.458 & 2449561.3525 & 10.112 & 0.993 & 0.624 & 0.672 & 1.450 \\
\hline 2449489.5518 & 10.137 & 1.013 & 0.655 & 0.674 & 1.466 & 2449563 & 10.100 & 0.997 & 0.627 & 0.662 & 1.439 \\
\hline 2449492.5127 & 10.110 & 1.010 & 0.649 & 0.666 & 1.459 & 2449575.4209 & 10.155 & 1.011 & 0.673 & 0.674 & 1.462 \\
\hline 2449495.5205 & 10.136 & 1.008 & 0.617 & 0.672 & 1.467 & 2449587.3325 & 10.186 & 1.032 & 0.696 & 0.683 & 1.483 \\
\hline 2449497.5693 & 10.139 & 0.995 & 0.627 & 0.673 & 1.472 & 2449590.3867 & 10.143 & 1.011 & 0.684 & 0.683 & 1.472 \\
\hline 2449502.5557 & 10.201 & 1.005 & 0.663 & 0.667 & 1.438 & 2449595.2964 & 10.116 & 0.996 & 0.685 & 0.660 & 1.454 \\
\hline 2449510.5547 & 10.215 & 1.022 & 0.684 & 0.683 & 1.484 & 2449598.3110 & 10.122 & 0.982 & 0.612 & 0.663 & 1.441 \\
\hline 2449513.4912 & 10.196 & 1.016 & 0.667 & 0.690 & 1.491 & 2449603.2734 & 10.147 & 1.023 & 0.624 & 0.675 & 1.466 \\
\hline 2449515.4893 & 10.196 & 1.016 & 0.668 & 0.687 & 1.485 & 2449607.2588 & 10.165 & 1.003 & 0.657 & 0.679 & 1.482 \\
\hline 2449518.5161 & 10.200 & 1.028 & 0.672 & 0.686 & 1.493 & 2449621.2637 & 10.187 & 1.029 & 0.660 & 0.688 & 1.499 \\
\hline 2449534.4951 & 10.148 & 1.004 & 0.655 & 0.671 & 1.461 & 2449624.2871 & 10.185 & 1.018 & 0.679 & 0.684 & 1.489 \\
\hline 2449538.5215 & 10.177 & 1.014 & 0.648 & 0.674 & 1.465 & 2449629.2793 & 10.149 & 1.021 & 0.655 & 0.687 & 1.490 \\
\hline 2449539.4331 & 10.174 & 1.015 & 0.651 & 0.677 & 1.472 & 2451626.6389 & 10.125 & 1.007 & 0.595 & 0.675 & 1.457 \\
\hline 2449544.4565 & 10.196 & 1.018 & 0.672 & 0.680 & 1.468 & 245 & 10.197 & 1.018 & 0.660 & 0.684 & 1.492 \\
\hline 2449547.4580 & 10.197 & 1.014 & 0.665 & 0.688 & 1.482 & 2451819.3136 & 10.191 & 1.023 & 0.646 & 0.685 & 1.482 \\
\hline \multicolumn{12}{|c|}{ IRAS 19157-0247 } \\
\hline 2448404.634 & .740 & 0.856 & 0.590 & 0.530 & 1.115 & 2449147.5633 & 10.674 & 0.802 & 0.535 & 0.516 & 1.061 \\
\hline 2448406.616 & 10.740 & 0.854 & 0.594 & 0.535 & 1.111 & 2449170.4974 & 10.740 & 0.800 & 0.524 & 0.499 & 1.042 \\
\hline 2448408.640 & 10.719 & 0.843 & 0.580 & 0.515 & 1.087 & 2449183.5664 & 10.689 & 0.801 & 0.487 & 0.509 & 1.062 \\
\hline 2448409.557 & 10.703 & 0.823 & 0.547 & 0.519 & 1.080 & 2449188.5495 & 10.694 & 0.815 & 0.522 & 0.518 & 1.077 \\
\hline 2448412.583 & 10.672 & 0.800 & 0.514 & 0.501 & 1.045 & 2449207.4435 & 10.624 & 0.774 & 0.464 & 0.498 & 1.036 \\
\hline 2448417.533 & 10.668 & 0.806 & 0.540 & 0.512 & 1.073 & 2449213.3872 & 10.733 & 0.817 & 0.529 & 0.512 & 1.066 \\
\hline 2448419.530 & 10.672 & 0.814 & 0.551 & 0.512 & 1.070 & 2449228.2715 & 10.699 & 0.840 & 0.542 & 0.517 & 1.098 \\
\hline 2448427.484 & 10.720 & 0.817 & 0.586 & 0.509 & 1.073 & 2449232.3948 & 10.736 & 0.855 & 0.588 & 0.538 & 1.110 \\
\hline 2448434.514 & 10.696 & 0.818 & 0.535 & 0.507 & 1.082 & 2449244.3211 & 10.700 & 0.806 & 0.527 & 0.510 & 1.062 \\
\hline 2448435.486 & 10.703 & 0.818 & 0.512 & 0.517 & 1.087 & 2449245.3233 & 10.680 & 0.806 & 0.534 & 0.510 & 1.059 \\
\hline 2448436.507 & 10.727 & 0.823 & 0.527 & 0.516 & 1.083 & 2451626.6478 & 10.662 & 0.876 & 0.571 & 0.508 & 1.065 \\
\hline 2448437.490 & 10.720 & 0.810 & 0.535 & 0.518 & 1.082 & 2451692.6036 & 10.659 & 0.798 & 0.544 & 0.522 & 1.067 \\
\hline 2448454.465 & 10.688 & 0.825 & 0.559 & 0.521 & 1.088 & 2451746.4517 & 10.713 & 0.804 & 0.516 & 0.508 & 1.056 \\
\hline 2448456.432 & 10.674 & 0.806 & 0.516 & 0.514 & 1.081 & 2451747.4226 & 10.710 & 0.800 & 0.500 & 0.502 & 1.052 \\
\hline 2448457.433 & 10.669 & 0.816 & 0.516 & 0.514 & 1.067 & 2451751.4427 & 10.697 & 0.811 & 0.504 & 0.511 & 1.062 \\
\hline 2448462.416 & 10.650 & 0.799 & 0.496 & 0.509 & 1.065 & 2451752.5005 & 10.724 & 0.828 & 0.532 & 0.523 & 1.077 \\
\hline 2448480.470 & 10.726 & 0.801 & 0.511 & 0.509 & 1.083 & 2451761.4192 & 10.753 & 0.852 & 0.555 & 0.540 & 1.121 \\
\hline 2448483.370 & 10.698 & 0.807 & 0.511 & 0.503 & 1.062 & 2451762.3703 & 10.736 & 0.844 & 0.555 & 0.525 & 1.097 \\
\hline
\end{tabular}


H. Van Winckel et al.: Binarity of post-AGB stars, Online Material p 8

Table 5. continued.

\begin{tabular}{clllllllllll}
\hline \hline HJD & $V$ & $B-V$ & $U-B$ & $V-R$ & $V-I$ & HJD & $V$ & $B-V$ & $U-B$ & $V-R$ & $V-I$ \\
\hline 2448500.383 & 10.696 & 0.819 & 0.538 & 0.518 & 1.089 & 2451772.3775 & 10.729 & 0.840 & 0.529 & 0.511 & 1.077 \\
2448511.324 & 10.719 & 0.813 & 0.518 & 0.522 & 1.087 & 2451773.3774 & 10.736 & 0.835 & 0.524 & 0.511 & 1.077 \\
2448799.5644 & 10.692 & 0.810 & 0.540 & 0.516 & 1.088 & 2451782.3920 & 10.664 & 0.820 & 0.534 & 0.511 & 1.072 \\
2448828.4964 & 10.630 & 0.806 & 0.534 & 0.507 & 1.072 & 2451784.3776 & 10.650 & 0.803 & 0.503 & 0.508 & 1.057 \\
2448843.3588 & 10.672 & 0.808 & 0.541 & 0.516 & 1.084 & 2451786.3757 & 10.682 & 0.801 & 0.498 & 0.505 & 1.049 \\
2449138.5385 & 10.708 & 0.815 & 0.563 & 0.524 & 1.085 & 2451816.2680 & 10.739 & 0.824 & 0.565 & 0.525 & 1.091 \\
2449146.5408 & 10.644 & 0.793 & 0.510 & 0.511 & 1.067 & 2451819.3207 & 10.725 & 0.846 & 0.547 & 0.524 & 1.092 \\
\hline
\end{tabular}


H. Van Winckel et al.: Binarity of post-AGB stars, Online Material p 9

Table 6. Individual photometric datapoints of the programme stars obtained with the Geneva P7 photometer, both on the Swiss telescope at La Silla and on the Flemish $1.2 \mathrm{~m}$ Mercator telescope.

\begin{tabular}{|c|c|c|c|c|c|c|c|c|c|}
\hline$\overline{\mathrm{HJD}(24+)}$ & 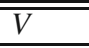 & {$[\overline{[U-B]}$} & 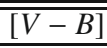 & $\overline{[\overline{[B 1-B]}}$ & $\overline{[\overline{[B 2-B]}}$ & {$[\overline{[V 1-B]}$} & 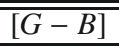 & "Weight(mag) & "Weight(colour) \\
\hline \multicolumn{10}{|c|}{ IRAS $12222-4652$} \\
\hline 47258.707 & 7.930 & 1.961 & 0.451 & 0.993 & 1.377 & 1.177 & 1.509 & 2 & 2 \\
\hline 47261.651 & 7.943 & 1.952 & 0.454 & 0.996 & 1.380 & 1.186 & 1.516 & 3 & 3 \\
\hline 47264.569 & 7.934 & 1.959 & 0.460 & 0.989 & 1.386 & 1.192 & 1.547 & 2 & 3 \\
\hline 47266.642 & 7.882 & 1.959 & 0.473 & 0.986 & 1.385 & 1.206 & 1.547 & 1 & 0 \\
\hline 47269.556 & 7.931 & 1.946 & 0.453 & 0.988 & 1.389 & 1.183 & 1.525 & 3 & 3 \\
\hline 47273.552 & 7.920 & 1.954 & 0.462 & 0.989 & 1.392 & 1.195 & 1.535 & 3 & 3 \\
\hline 47276.551 & 7.922 & 1.946 & 0.457 & 0.995 & 1.389 & 1.190 & 1.529 & 3 & 3 \\
\hline 47282.624 & 7.930 & 1.941 & 0.446 & 0.988 & 1.390 & 1.183 & 1.522 & 3 & 3 \\
\hline 47284.625 & 7.924 & 1.960 & 0.457 & 0.998 & 1.388 & 1.183 & 1.528 & 3 & 3 \\
\hline 47289.644 & 7.919 & 1.946 & 0.474 & 0.984 & 1.392 & 1.207 & 1.546 & 2 & 3 \\
\hline 47296.656 & 7.958 & 1.951 & 0.477 & 0.990 & 1.386 & 1.211 & 1.549 & 0 & 2 \\
\hline 47304.565 & 7.905 & 1.958 & 0.472 & 0.992 & 1.393 & 1.207 & 1.547 & 3 & 3 \\
\hline 47308.600 & 7.916 & 1.977 & 0.462 & 0.995 & 1.389 & 1.200 & 1.533 & 3 & 3 \\
\hline 47315.589 & 7.944 & 1.952 & 0.454 & 0.993 & 1.395 & 1.183 & 1.525 & 3 & 3 \\
\hline 47493.816 & 7.894 & 1.939 & 0.477 & 0.989 & 1.396 & 1.211 & 1.552 & 3 & 3 \\
\hline 47495.814 & 7.896 & 1.934 & 0.472 & 0.979 & 1.393 & 1.197 & 1.548 & 3 & 3 \\
\hline 47505.839 & 7.906 & 1.946 & 0.468 & 0.985 & 1.383 & 1.204 & 1.549 & 3 & 4 \\
\hline 47519.850 & 7.932 & 1.963 & 0.440 & 0.990 & 1.383 & 1.173 & 1.512 & 3 & 3 \\
\hline 47521.832 & 7.945 & 1.954 & 0.432 & 0.995 & 1.368 & 1.174 & 1.501 & 3 & 3 \\
\hline 47537.845 & 7.935 & 1.973 & 0.456 & 0.996 & 1.388 & 1.187 & 1.532 & 4 & 4 \\
\hline 47540.844 & 7.945 & 1.962 & 0.447 & 0.999 & 1.380 & 1.177 & 1.521 & 4 & 4 \\
\hline 47542.844 & 7.938 & 1.983 & 0.447 & 0.997 & 1.382 & 1.183 & 1.524 & 3 & 3 \\
\hline 47544.821 & 7.934 & 1.981 & 0.441 & 1.000 & 1.375 & 1.180 & 1.512 & 3 & 3 \\
\hline 47546.849 & 7.928 & 1.982 & 0.457 & 1.003 & 1.387 & 1.183 & 1.529 & 3 & 3 \\
\hline 47549.823 & 7.945 & 1.967 & 0.447 & 0.998 & 1.387 & 1.184 & 1.521 & 3 & 3 \\
\hline 47551.838 & 7.915 & 1.974 & 0.463 & 0.995 & 1.384 & 1.197 & 1.532 & 3 & 3 \\
\hline 47596.748 & 7.944 & 1.964 & 0.439 & 1.001 & 1.376 & 1.170 & 1.502 & 3 & 3 \\
\hline 47598.772 & 7.941 & 1.966 & 0.439 & 1.003 & 1.369 & 1.175 & 1.511 & 3 & 3 \\
\hline 47600.764 & 7.937 & 1.947 & 0.450 & 0.998 & 1.382 & 1.183 & 1.516 & 3 & 3 \\
\hline 47601.733 & 7.928 & 1.956 & 0.463 & 0.996 & 1.390 & 1.197 & 1.526 & 2 & 3 \\
\hline 47602.692 & 7.916 & 1.959 & 0.457 & 0.990 & 1.388 & 1.183 & 1.534 & 3 & 3 \\
\hline 47605.675 & 7.928 & 1.976 & 0.458 & 0.991 & 1.393 & 1.189 & 1.526 & 3 & 4 \\
\hline 47606.673 & 7.936 & 1.961 & 0.455 & 0.987 & 1.382 & 1.185 & 1.527 & 3 & 3 \\
\hline 47607.669 & 7.932 & 1.953 & 0.457 & 0.985 & 1.383 & 1.187 & 1.530 & 2 & 2 \\
\hline 47608.714 & 7.925 & 1.952 & 0.460 & 0.986 & 1.385 & 1.189 & 1.522 & 3 & 3 \\
\hline 47609.733 & 7.913 & 1.962 & 0.461 & 0.995 & 1.387 & 1.192 & 1.540 & 2 & 3 \\
\hline 47610.678 & 7.914 & 1.946 & 0.468 & 0.985 & 1.388 & 1.198 & 1.537 & 2 & 3 \\
\hline 47611.683 & 7.914 & 1.945 & 0.458 & 0.989 & 1.389 & 1.189 & 1.528 & 2 & 3 \\
\hline 47612.650 & 7.922 & 1.960 & 0.464 & 0.997 & 1.390 & 1.194 & 1.529 & 2 & 2 \\
\hline 47613.630 & 7.908 & 1.946 & 0.463 & 0.985 & 1.393 & 1.202 & 1.544 & 2 & 3 \\
\hline 47614.616 & 7.940 & 1.960 & 0.444 & 0.994 & 1.387 & 1.181 & 1.522 & 2 & 2 \\
\hline 47636.622 & 7.917 & 1.926 & 0.469 & 0.988 & 1.391 & 1.201 & 1.539 & 3 & 3 \\
\hline 47637.557 & 7.904 & 1.931 & 0.472 & 0.989 & 1.396 & 1.200 & 1.546 & 3 & 3 \\
\hline 47638.572 & 7.898 & 1.935 & 0.462 & 0.991 & 1.391 & 1.198 & 1.545 & 3 & 3 \\
\hline 47639.559 & 7.903 & 1.935 & 0.467 & 0.988 & 1.383 & 1.195 & 1.539 & 3 & 3 \\
\hline 47640.572 & 7.889 & 1.935 & 0.467 & 0.984 & 1.392 & 1.208 & 1.549 & 3 & 3 \\
\hline 47643.654 & 7.900 & 1.950 & 0.472 & 0.979 & 1.390 & 1.207 & 1.549 & 3 & 3 \\
\hline 47648.573 & 7.894 & 1.955 & 0.474 & 0.986 & 1.394 & 1.204 & 1.553 & 2 & 3 \\
\hline 47655.605 & 7.930 & 1.962 & 0.459 & 0.986 & 1.394 & 1.190 & 1.535 & 3 & 3 \\
\hline 47656.599 & 7.934 & 1.961 & 0.455 & 0.988 & 1.383 & 1.190 & 1.529 & 3 & 3 \\
\hline 47881.838 & 7.984 & 1.974 & 0.425 & 1.003 & 1.366 & 1.158 & 1.490 & 2 & 3 \\
\hline 47883.832 & 7.973 & 1.941 & 0.429 & 1.011 & 1.373 & 1.163 & 1.493 & 3 & 3 \\
\hline 47885.829 & 7.978 & 1.943 & 0.429 & 1.005 & 1.380 & 1.163 & 1.494 & 3 & 3 \\
\hline 47887.830 & 7.979 & 1.962 & 0.418 & 1.008 & 1.372 & 1.154 & 1.487 & 2 & 2 \\
\hline 47892.827 & 7.957 & 1.950 & 0.425 & 0.992 & 1.374 & 1.159 & 1.493 & 3 & 3 \\
\hline 47893.821 & 7.945 & 1.967 & 0.436 & 1.000 & 1.377 & 1.173 & 1.504 & 3 & 3 \\
\hline 47896.787 & 7.944 & 1.978 & 0.440 & 1.006 & 1.379 & 1.175 & 1.510 & 3 & 3 \\
\hline 47898.826 & 7.932 & 1.969 & 0.456 & 0.995 & 1.378 & 1.185 & 1.522 & 3 & 3 \\
\hline 47901.846 & 7.935 & 1.954 & 0.465 & 0.993 & 1.392 & 1.198 & 1.541 & 3 & 3 \\
\hline 47902.836 & 7.930 & 1.969 & 0.465 & 0.991 & 1.381 & 1.200 & 1.535 & 3 & 3 \\
\hline 47903.848 & 7.933 & 1.976 & 0.468 & 0.989 & 1.395 & 1.201 & 1.541 & 3 & 3 \\
\hline 48701.675 & 8.012 & 1.943 & 0.417 & 1.011 & 1.369 & 1.153 & 1.484 & 2 & 2 \\
\hline
\end{tabular}


Table 6. continued.

\begin{tabular}{|c|c|c|c|c|c|c|c|c|c|}
\hline HJD(24+) & $\bar{V}$ & $\overline{[U-B]}$ & $\overline{[}[V-B]$ & $\overline{[B 1-B]}$ & {$[\overline{[B 2-B]}$} & {$[V 1-B]$} & {$[G-B]$} & Weight(mag) & Weight(colour) \\
\hline 48704.747 & 8.009 & 1.952 & 0.421 & 1.011 & 1.379 & 1.154 & 1.491 & 3 & 3 \\
\hline 48706.648 & 8.010 & 1.950 & 0.423 & 1.012 & 1.367 & 1.159 & 1.490 & 2 & 2 \\
\hline 48735.615 & 7.893 & 1.980 & 0.474 & 0.979 & 1.392 & 1.208 & 1.548 & 2 & 3 \\
\hline 48753.660 & 8.017 & 1.956 & 0.430 & 1.006 & 1.370 & 1.157 & 1.500 & 2 & 2 \\
\hline 48794.490 & 7.944 & 1.970 & 0.455 & 0.992 & 1.393 & 1.192 & 1.520 & 3 & 3 \\
\hline 49043.782 & 7.965 & 1.922 & 0.457 & 0.990 & 1.380 & 1.192 & 1.530 & 3 & 3 \\
\hline 49050.771 & 7.923 & 1.944 & 0.473 & 0.984 & 1.397 & 1.200 & 1.542 & 3 & 3 \\
\hline 49057.794 & 7.879 & 1.951 & 0.477 & 0.981 & 1.392 & 1.212 & 1.549 & 3 & 3 \\
\hline 49063.785 & 7.874 & 1.951 & 0.479 & 0.981 & 1.396 & 1.214 & 1.553 & 3 & 3 \\
\hline 49085.668 & 7.967 & 1.946 & 0.448 & 0.986 & 1.382 & 1.185 & 1.523 & 2 & 3 \\
\hline 49095.594 & 7.938 & 1.943 & 0.452 & 0.992 & 1.391 & 1.176 & 1.518 & 2 & 2 \\
\hline 49100.617 & 7.931 & 1.944 & 0.463 & 0.993 & 1.387 & 1.188 & 1.523 & 2 & 2 \\
\hline 49102.615 & 7.929 & 1.939 & 0.466 & 0.986 & 1.389 & 1.201 & 1.536 & 3 & 3 \\
\hline 49104.622 & 7.907 & 1.947 & 0.474 & 0.986 & 1.391 & 1.206 & 1.550 & 3 & 3 \\
\hline 49105.646 & 7.907 & 1.943 & 0.480 & 0.977 & 1.382 & 1.213 & 1.554 & 2 & 3 \\
\hline 49386.836 & 7.865 & 1.959 & 0.487 & 0.978 & 1.394 & 1.214 & 1.559 & 2 & 3 \\
\hline 49407.783 & 7.967 & 1.960 & 0.452 & 0.996 & 1.387 & 1.182 & 1.520 & 3 & 3 \\
\hline 49414.851 & 7.993 & 1.937 & 0.422 & 1.001 & 1.367 & 1.160 & 1.487 & 3 & 3 \\
\hline 49426.796 & 7.966 & 1.921 & 0.421 & 0.997 & 1.373 & 1.159 & 1.487 & 3 & 3 \\
\hline 49451.758 & 7.913 & 1.951 & 0.480 & 0.978 & 1.395 & 1.209 & 1.558 & 3 & 3 \\
\hline 49454.771 & 7.935 & 1.935 & 0.469 & 0.988 & 1.388 & 1.202 & 1.536 & 3 & 3 \\
\hline 49456.751 & 7.942 & 1.941 & 0.458 & 0.990 & 1.381 & 1.188 & 1.529 & 3 & 3 \\
\hline 49459.723 & 7.965 & 1.955 & 0.434 & 0.992 & 1.372 & 1.166 & 1.505 & 3 & 3 \\
\hline 49460.733 & 7.970 & 1.939 & 0.433 & 1.000 & 1.384 & 1.168 & 1.498 & 2 & 2 \\
\hline 49468.725 & 7.976 & 1.941 & 0.437 & 1.003 & 1.381 & 1.170 & 1.500 & 3 & 3 \\
\hline 49513.584 & 7.931 & 1.940 & 0.446 & 0.995 & 1.383 & 1.175 & 1.513 & 3 & 3 \\
\hline 49521.566 & 7.929 & 1.936 & 0.454 & 0.990 & 1.387 & 1.186 & 1.519 & 3 & 3 \\
\hline 49524.543 & 7.937 & 1.940 & 0.452 & 0.994 & 1.392 & 1.194 & 1.522 & 4 & 3 \\
\hline 49794.742 & 7.937 & 1.968 & 0.469 & 1.001 & 1.387 & 1.199 & 1.536 & 2 & 3 \\
\hline 49796.724 & 7.935 & 1.969 & 0.445 & 0.996 & 1.385 & 1.183 & 1.518 & 2 & 2 \\
\hline 49798.764 & 7.950 & 1.948 & 0.447 & 0.991 & 1.386 & 1.179 & 1.518 & 3 & 3 \\
\hline 49800.749 & 7.965 & 1.961 & 0.449 & 0.997 & 1.382 & 1.179 & 1.515 & 3 & 3 \\
\hline 49802.707 & 7.968 & 1.960 & 0.448 & 0.997 & 1.385 & 1.179 & 1.518 & 3 & 2 \\
\hline 49806.613 & 7.958 & 1.946 & 0.441 & 0.995 & 1.379 & 1.181 & 1.515 & 3 & 2 \\
\hline 49808.725 & 7.962 & 1.949 & 0.433 & 0.996 & 1.376 & 1.173 & 1.500 & 2 & 1 \\
\hline 49831.651 & 7.898 & 1.948 & 0.485 & 0.976 & 1.395 & 1.216 & 1.560 & 2 & 3 \\
\hline 49833.676 & 7.899 & 1.954 & 0.487 & 0.980 & 1.395 & 1.220 & 1.559 & 3 & 3 \\
\hline 49836.630 & 7.904 & 1.964 & 0.487 & 0.974 & 1.394 & 1.217 & 1.554 & 3 & 2 \\
\hline 49839.679 & 7.911 & 1.965 & 0.484 & 0.982 & 1.390 & 1.214 & 1.558 & 3 & 3 \\
\hline 49841.517 & 7.928 & 1.965 & 0.485 & 0.981 & 1.397 & 1.210 & 1.554 & 3 & 3 \\
\hline 49846.663 & 7.939 & 1.952 & 0.476 & 0.996 & 1.401 & 1.207 & 1.543 & 3 & 3 \\
\hline 49851.661 & 7.970 & 1.939 & 0.449 & 0.995 & 1.386 & 1.189 & 1.525 & 3 & 3 \\
\hline 49853.656 & 7.966 & 1.954 & 0.451 & 0.994 & 1.384 & 1.183 & 1.520 & 3 & 2 \\
\hline 50109.846 & 8.002 & 1.939 & 0.415 & 1.013 & 1.362 & 1.154 & 1.474 & 3 & 3 \\
\hline 50112.858 & 8.019 & 1.933 & 0.413 & 1.017 & 1.381 & 1.144 & 1.470 & 3 & 3 \\
\hline 50115.801 & 8.020 & 1.935 & 0.414 & 1.007 & 1.369 & 1.143 & 1.476 & 3 & 3 \\
\hline 50116.850 & 8.018 & 1.933 & 0.420 & 1.009 & 1.371 & 1.147 & 1.483 & 3 & 3 \\
\hline 50119.853 & 7.995 & 1.923 & 0.429 & 1.003 & 1.380 & 1.163 & 1.494 & 3 & 2 \\
\hline 50121.851 & 8.000 & 1.927 & 0.425 & 0.998 & 1.369 & 1.157 & 1.482 & 3 & 3 \\
\hline 50123.859 & 7.972 & 1.932 & 0.434 & 0.999 & 1.377 & 1.166 & 1.500 & 2 & 2 \\
\hline 50124.864 & 7.962 & 1.945 & 0.445 & 0.994 & 1.381 & 1.186 & 1.520 & 3 & 3 \\
\hline 50125.849 & 7.954 & 1.938 & 0.449 & 0.992 & 1.386 & 1.188 & 1.523 & 3 & 3 \\
\hline & & & & & EN TrA & & & & \\
\hline 47608.812 & 8.885 & 2.154 & -0.086 & 1.151 & 1.242 & 0.680 & 0.897 & 3 & 3 \\
\hline 47608.817 & 8.891 & 2.112 & -0.078 & 1.158 & 1.242 & 0.681 & 0.905 & 3 & 3 \\
\hline 47609.759 & 8.903 & 2.078 & -0.066 & 1.151 & 1.250 & 0.695 & 0.912 & 2 & 3 \\
\hline 47610.756 & 8.908 & 2.056 & -0.048 & 1.142 & 1.255 & 0.715 & 0.936 & 2 & 3 \\
\hline 47611.793 & 8.923 & 2.061 & -0.035 & 1.123 & 1.255 & 0.720 & 0.942 & 2 & 3 \\
\hline 47612.785 & 8.905 & 2.021 & -0.013 & 1.123 & 1.264 & 0.747 & 0.974 & 2 & 2 \\
\hline 47614.822 & 8.859 & 2.020 & 0.032 & 1.116 & 1.286 & 0.789 & 1.021 & 2 & 2 \\
\hline 47636.730 & 8.477 & 2.029 & 0.028 & 1.116 & 1.279 & 0.780 & 1.021 & 3 & 3 \\
\hline 47637.730 & 8.489 & 2.032 & 0.020 & 1.098 & 1.278 & 0.776 & 1.014 & 3 & 3 \\
\hline 47638.678 & 8.510 & 2.039 & 0.026 & 1.110 & 1.279 & 0.784 & 1.015 & 3 & 3 \\
\hline 47639.670 & 8.530 & 2.031 & 0.027 & 1.115 & 1.286 & 0.784 & 1.022 & 3 & 3 \\
\hline 47640.677 & 8.558 & 2.042 & 0.028 & 1.106 & 1.281 & 0.785 & 1.025 & 3 & 3 \\
\hline 47643.718 & 8.606 & 2.002 & 0.063 & 1.084 & 1.301 & 0.817 & 1.065 & 3 & 3 \\
\hline 47648.707 & 8.651 & 1.979 & 0.145 & 1.060 & 1.328 & 0.898 & 1.157 & 2 & 3 \\
\hline
\end{tabular}


Table 6. continued.

\begin{tabular}{|c|c|c|c|c|c|c|c|c|c|}
\hline HJD(24+) & $\bar{V}$ & $\overline{[U-B]}$ & $\overline{\overline{[V-B]}}$ & $\overline{\overline{[B 1-B]}}$ & $\overline{[\overline{[B 2-B]}}$ & $\overline{[\overline{[V 1-B]}}$ & $\overline{[G-B]}$ & "Weight(mag) & "Weight(colour) \\
\hline 47655.679 & 8.509 & 1.972 & 0.217 & 1.045 & 1.345 & 0.967 & 1.239 & 3 & 3 \\
\hline 47656.686 & 8.503 & 1.970 & 0.199 & 1.038 & 1.342 & 0.947 & 1.221 & 3 & 3 \\
\hline 48099.521 & 8.614 & 1.993 & 0.201 & 1.039 & 1.335 & 0.942 & 1.207 & 2 & 2 \\
\hline 48101.491 & 8.567 & 1.986 & 0.249 & 1.010 & 1.347 & 0.989 & 1.266 & 3 & 3 \\
\hline 48103.496 & 8.554 & 2.002 & 0.257 & 1.019 & 1.368 & 0.996 & 1.282 & 2 & 2 \\
\hline 48105.510 & 8.582 & 2.002 & 0.226 & 1.036 & 1.349 & 0.965 & 1.251 & 2 & 2 \\
\hline 48113.524 & 8.727 & 2.031 & -0.004 & 1.123 & 1.268 & 0.753 & 0.985 & 3 & 3 \\
\hline 48117.533 & 8.763 & 2.119 & -0.091 & 1.159 & 1.232 & 0.669 & 0.881 & 3 & 3 \\
\hline 48133.582 & 9.088 & 2.112 & -0.093 & 1.169 & 1.258 & 0.671 & 0.875 & 3 & 3 \\
\hline 48339.736 & 8.698 & 2.082 & -0.050 & 1.143 & 1.258 & 0.718 & 0.934 & 2 & 2 \\
\hline 48342.766 & 8.727 & 2.106 & -0.065 & 1.157 & 1.248 & 0.699 & 0.922 & 3 & 3 \\
\hline 48349.795 & 8.804 & 2.092 & -0.042 & 1.141 & 1.247 & 0.717 & 0.943 & 2 & 2 \\
\hline 48371.721 & 8.562 & 2.043 & 0.111 & 1.082 & 1.304 & 0.863 & 1.109 & 3 & 3 \\
\hline 48374.709 & 8.605 & 2.071 & 0.033 & 1.109 & 1.283 & 0.792 & 1.030 & 2 & 2 \\
\hline 48377.715 & 8.645 & 2.102 & -0.008 & 1.126 & 1.270 & 0.753 & 0.990 & 3 & 3 \\
\hline 48384.677 & 8.812 & 2.102 & -0.079 & 1.158 & 1.246 & 0.684 & 0.904 & 3 & 3 \\
\hline 48389.716 & 8.932 & 2.064 & -0.082 & 1.149 & 1.253 & 0.679 & 0.896 & 3 & 3 \\
\hline 48735.757 & 8.536 & 2.007 & 0.174 & 1.052 & 1.329 & 0.922 & 1.195 & 2 & 3 \\
\hline 48736.731 & 8.536 & 2.021 & 0.155 & 1.057 & 1.331 & 0.912 & 1.171 & 2 & 3 \\
\hline 48793.619 & 8.729 & 2.071 & -0.030 & 1.131 & 1.254 & 0.724 & 0.960 & 2 & 3 \\
\hline 49049.872 & 8.673 & 2.042 & -0.019 & 1.126 & 1.257 & 0.736 & 0.975 & 3 & 3 \\
\hline 49054.849 & 8.816 & 1.974 & 0.022 & 1.103 & 1.282 & 0.780 & 1.016 & 3 & 3 \\
\hline 49057.852 & 8.811 & 1.962 & 0.057 & 1.084 & 1.310 & 0.819 & 1.066 & 3 & 3 \\
\hline 49060.845 & 8.711 & 1.937 & 0.111 & 1.057 & 1.317 & 0.863 & 1.117 & 3 & 3 \\
\hline 49063.832 & 8.594 & 1.942 & 0.150 & 1.059 & 1.326 & 0.899 & 1.163 & 3 & 3 \\
\hline 49085.770 & 8.627 & 2.045 & 0.016 & 1.132 & 1.290 & 0.772 & 1.010 & 2 & 3 \\
\hline 49411.874 & 8.559 & 2.047 & -0.016 & 1.130 & 1.264 & 0.748 & 0.970 & 3 & 3 \\
\hline 49412.877 & 8.589 & 2.045 & -0.008 & 1.124 & 1.269 & 0.753 & 0.988 & 3 & 2 \\
\hline 49415.878 & 8.647 & 2.033 & 0.000 & 1.117 & 1.275 & 0.755 & 0.997 & 3 & 3 \\
\hline 49422.856 & 8.707 & 1.968 & 0.133 & 1.063 & 1.326 & 0.885 & 1.138 & 2 & 2 \\
\hline 49426.858 & 8.563 & 1.963 & 0.225 & 1.028 & 1.347 & 0.966 & 1.254 & 3 & 3 \\
\hline 49451.801 & 8.815 & 2.151 & -0.150 & 1.181 & 1.215 & 0.619 & 0.817 & 3 & 3 \\
\hline 49456.805 & 8.972 & 2.132 & -0.112 & 1.173 & 1.237 & 0.660 & 0.858 & 3 & 3 \\
\hline 49468.714 & 8.526 & 1.988 & 0.181 & 1.052 & 1.331 & 0.942 & 1.198 & 3 & 3 \\
\hline 49513.624 & 8.528 & 2.078 & -0.002 & 1.122 & 1.263 & 0.757 & 0.992 & 3 & 3 \\
\hline 49521.676 & 8.646 & 2.113 & -0.111 & 1.173 & 1.235 & 0.655 & 0.872 & 3 & 3 \\
\hline 49797.816 & 8.589 & 1.996 & 0.221 & 1.032 & 1.352 & 0.977 & 1.248 & 3 & 3 \\
\hline 49800.806 & 8.617 & 2.022 & 0.171 & 1.044 & 1.332 & 0.928 & 1.179 & 3 & 3 \\
\hline 49803.814 & 8.654 & 2.052 & 0.080 & 1.086 & 1.306 & 0.837 & 1.087 & 2 & 2 \\
\hline 49807.796 & 8.649 & 2.050 & 0.008 & 1.117 & 1.276 & 0.772 & 0.999 & 3 & 3 \\
\hline 49831.731 & 8.647 & 1.995 & 0.200 & 1.042 & 1.341 & 0.959 & 1.219 & 2 & 3 \\
\hline 49837.720 & 8.665 & 1.986 & 0.102 & 1.073 & 1.308 & 0.870 & 1.115 & 3 & 2 \\
\hline 49839.734 & 8.682 & 2.008 & 0.058 & 1.092 & 1.298 & 0.817 & 1.049 & 3 & 3 \\
\hline 49841.720 & 8.695 & 2.022 & 0.013 & 1.108 & 1.281 & 0.770 & 1.001 & 3 & 3 \\
\hline 49846.695 & 8.715 & 2.110 & -0.077 & 1.162 & 1.250 & 0.688 & 0.902 & 3 & 3 \\
\hline 49851.684 & 8.799 & 2.052 & -0.117 & 1.170 & 1.240 & 0.648 & 0.859 & 3 & 3 \\
\hline 49853.672 & 8.830 & 2.057 & -0.119 & 1.165 & 1.237 & 0.644 & 0.865 & 3 & 2 \\
\hline \multicolumn{10}{|c|}{ IRAS $19125+0343$} \\
\hline 52415.66975 & 10.230 & 2.381 & -0.163 & 1.033 & 1.323 & 0.610 & 0.791 & 3 & 3 \\
\hline 524317.69291 & 10.238 & 2.387 & -0.153 & 1.039 & 1.338 & 0.622 & 0.799 & 3 & 3 \\
\hline 524219.69850 & 10.233 & 2.399 & -0.167 & 1.026 & 1.315 & 0.609 & 0.784 & 2 & 2 \\
\hline 524321.66363 & 10.268 & 2.372 & -0.164 & 1.036 & 1.323 & 0.615 & 0.773 & 2 & 3 \\
\hline 524322.68742 & 10.269 & 2.360 & -0.173 & 1.029 & 1.303 & 0.600 & 0.775 & 3 & 3 \\
\hline 524327.67544 & 10.283 & 2.396 & -0.178 & 1.042 & 1.317 & 0.602 & 0.761 & 2 & 3 \\
\hline 524328.67778 & 10.289 & 2.409 & -0.177 & 1.050 & 1.318 & 0.598 & 0.771 & 3 & 3 \\
\hline 524329.68165 & 10.286 & 2.418 & -0.176 & 1.041 & 1.320 & 0.601 & 0.766 & 2 & 3 \\
\hline 524251.60071 & 10.234 & 2.426 & -0.159 & 1.034 & 1.333 & 0.613 & 0.799 & 3 & 2 \\
\hline 524352.60556 & 10.234 & 2.421 & -0.163 & 1.021 & 1.332 & 0.624 & 0.794 & 2 & 3 \\
\hline 524253.62684 & 10.242 & 2.442 & -0.161 & 1.038 & 1.324 & 0.614 & 0.798 & 2 & 2 \\
\hline 524354.62246 & 10.255 & 2.460 & -0.156 & 1.043 & 1.339 & 0.612 & 0.794 & 3 & 3 \\
\hline 524355.62875 & 10.247 & 2.419 & -0.161 & 1.040 & 1.317 & 0.608 & 0.779 & 2 & 3 \\
\hline 524256.61663 & 10.258 & 2.427 & -0.167 & 1.056 & 1.334 & 0.606 & 0.787 & 3 & 2 \\
\hline 524358.61192 & 10.273 & 2.413 & -0.164 & 1.044 & 1.324 & 0.598 & 0.784 & 2 & 3 \\
\hline 524376.52627 & 10.214 & 2.427 & -0.181 & 1.030 & 1.312 & 0.608 & 0.786 & 3 & 3 \\
\hline 524288.51420 & 10.216 & 2.413 & -0.160 & 1.030 & 1.335 & 0.606 & 0.799 & 2 & 2 \\
\hline
\end{tabular}


H. Van Winckel et al.: Binarity of post-AGB stars, Online Material p 12

Table 6. continued.

\begin{tabular}{|c|c|c|c|c|c|c|c|c|c|}
\hline "HJD(24+) & $\overline{\bar{V}}$ & $\overline{[\overline{[U-B]}}$ & $\overline{[\overline{[V-B]}}$ & $\overline{\overline{[B B 1-B]}}$ & 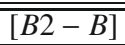 & {$[V 1-B]$} & $\overline{[\overline{[G-B]}}$ & Weight(mag) & Weight(colour) \\
\hline 524391.52369 & 10.223 & 2.407 & -0.156 & 1.047 & 1.334 & 0.617 & 0.796 & 2 & 3 \\
\hline 524392.47117 & 10.232 & 2.385 & -0.159 & 1.045 & 1.333 & 0.625 & 0.797 & 3 & 3 \\
\hline 524293.49170 & 10.234 & 2.400 & -0.160 & 1.031 & 1.342 & 0.623 & 0.796 & 3 & 2 \\
\hline 524399.46539 & 10.259 & 2.388 & -0.167 & 1.037 & 1.333 & 0.607 & 0.785 & 2 & 3 \\
\hline 525301.45406 & 10.260 & 2.405 & -0.170 & 1.030 & 1.307 & 0.610 & 0.781 & 2 & 3 \\
\hline 525305.47919 & 10.275 & 2.456 & -0.168 & 1.040 & 1.327 & 0.606 & 0.777 & 3 & 3 \\
\hline 525306.47941 & 10.268 & 2.516 & -0.178 & 1.039 & 1.325 & 0.590 & 0.771 & 3 & 3 \\
\hline 525330.42345 & 10.214 & 2.402 & -0.161 & 1.030 & 1.337 & 0.621 & 0.786 & 2 & 3 \\
\hline 525261.38422 & 10.218 & 2.385 & -0.162 & 1.045 & 1.343 & 0.617 & 0.794 & 2 & 2 \\
\hline 528319.61978 & 10.219 & 2.359 & -0.137 & 1.024 & 1.331 & 0.628 & 0.808 & 3 & 3 \\
\hline 528322.61384 & 10.220 & 2.360 & -0.134 & 1.019 & 1.338 & 0.631 & 0.829 & 3 & 3 \\
\hline 528325.61868 & 10.230 & 2.361 & -0.136 & 1.022 & 1.339 & 0.632 & 0.816 & 2 & 3 \\
\hline 528352.54597 & 10.243 & 2.464 & -0.160 & 1.052 & 1.332 & 0.615 & 0.788 & 3 & 3 \\
\hline 528353.50173 & 10.261 & 2.452 & -0.156 & 1.042 & 1.332 & 0.614 & 0.788 & 3 & 3 \\
\hline 528354.48603 & 10.249 & 2.448 & -0.167 & 1.054 & 1.330 & 0.620 & 0.787 & 2 & 3 \\
\hline 528456.50272 & 10.240 & 2.453 & -0.162 & 1.046 & 1.325 & 0.606 & 0.790 & 3 & 4 \\
\hline 535390.49430 & 10.244 & 2.412 & -0.142 & 1.033 & 1.335 & 0.630 & 0.807 & 2 & 3 \\
\hline 535393.51927 & 10.233 & 2.423 & -0.154 & 1.038 & 1.350 & 0.621 & 0.805 & 2 & 3 \\
\hline 535394.52526 & 10.247 & 2.395 & -0.149 & 1.038 & 1.334 & 0.623 & 0.808 & 4 & 3 \\
\hline 535395.52349 & 10.242 & 2.380 & -0.153 & 1.035 & 1.328 & 0.627 & 0.798 & 4 & 3 \\
\hline 536206.48002 & 10.265 & 2.393 & -0.169 & 1.023 & 1.328 & 0.601 & 0.782 & 2 & 2 \\
\hline \multicolumn{10}{|c|}{ IRAS 19157-0247 } \\
\hline 52446.63893 & 10.725 & 2.290 & -0.015 & 1.006 & 1.332 & 0.751 & 0.964 & 1 & 3 \\
\hline 52501.50119 & 10.742 & 2.339 & -0.051 & 1.033 & 1.351 & 0.712 & 0.911 & 2 & 3 \\
\hline 52502.46879 & 10.749 & 2.351 & -0.060 & 1.026 & 1.356 & 0.713 & 0.903 & 2 & 3 \\
\hline 52505.44584 & 10.775 & 2.332 & -0.056 & 1.026 & 1.346 & 0.716 & 0.921 & 3 & 3 \\
\hline 52507.43737 & 10.753 & 2.290 & -0.041 & 1.012 & 1.358 & 0.722 & 0.941 & 2 & 1 \\
\hline 52561.39041 & 10.688 & 2.278 & -0.002 & 1.004 & 1.368 & 0.778 & 0.969 & 3 & 3 \\
\hline
\end{tabular}

